Supporting Information

\title{
Gold-Catalyzed Assembly of Heterobicyclic Systems
}

Liming Zhang and Sergey A. Kozmin

University of Chicago, Department of Chemistry, 5735 South Ellis Ave., Chicago, IL 60637 
General. Ethyl acetate (ACS grade), hexanes (ACS grade) and diethyl ether (ACS grade) were purchased from Fisher Scientific and used without further purification. Anhydrous dichloromethane (HPLC grade) was purified by distillation over calcium hydride. Anhydrous tetrahydrofuran was freshly distilled from sodium-benzophenone. Commercially available reagents were used without further purification. Reactions were monitored by thin layer chromatography ( TLC) using whatman precoated silica gel plates. Flash column chromatography was performed over silacycle silica gel (230-400 mesh). ${ }^{1} \mathrm{H}$ NMR and ${ }^{13} \mathrm{C}$ NMR spectra were recorded on Bruker DMX-500 spectrometers using residue solvent peaks as an internal standard. Infrared spectra were recorded with a Nicolet FTIR spectrometer and are reported in reciprocal centimeter $\left(\mathrm{cm}^{-1}\right)$. Mass spectra were recorded with a Varian Saturn 2000 GC-MS using EI method or an Agilent 1100 LCMS using APCI or ES methods.

\section{Preparation of Enynes}
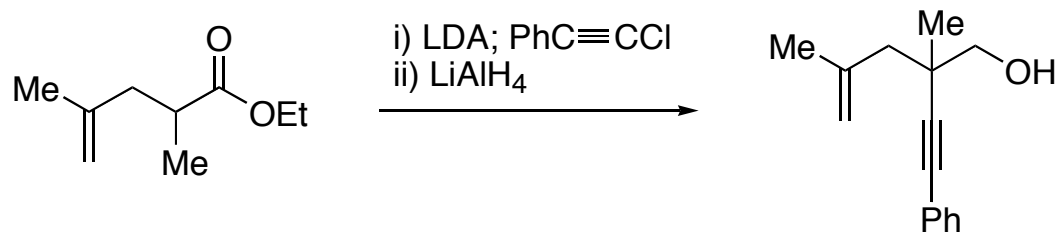

1

Enyne 1. The title compound was prepared by using a procedure modified from that of Kende and co-workers. ${ }^{1}$ To a solution of LDA in THF $(10 \mathrm{~mL})$, generated by the treatment of diisopropylamine $(0.46 \mathrm{~mL}, 3.3 \mathrm{mmol})$ with $2.5 \mathrm{M} \mathrm{n}$-BuLi in hexanes $(1.32 \mathrm{~mL}, 3.3 \mathrm{mmol})$ and cooled in a dry ice-acetone bath, was added a solution of ethyl 2,4-dimethylpent-4-enoate $(0.515$ $\mathrm{g}, 3.3 \mathrm{mmol})$ in THF $(2 \mathrm{~mL})$. Upon completion, HMPA $(0.574 \mathrm{~mL}, 3.3 \mathrm{mmol})$ was added. The enolate solution was stirred at the same temperature for $30 \mathrm{~min}$ before a solution of phenyl chloroacetylene $(0.41 \mathrm{~g}, 3 \mathrm{mmol})$ in THF $(2 \mathrm{~mL})$ was added. The reaction was allowed to warm to room temperature overnight and then poured into water and extracted with $\mathrm{Et}_{2} \mathrm{O}(3 \times 20 \mathrm{~mL})$. The combined organic layers were washed with $\mathrm{H}_{2} \mathrm{O}$ and brine, dried $\left(\mathrm{MgSO}_{4}\right)$, and concentrated. The residue was purified through silica gel flash chromatography to yield the alkynyl ester. At $0^{\circ} \mathrm{C}$, to a solution of the alkynyl ester obtained above in THF $(30 \mathrm{~mL})$ was added $\mathrm{LiAlH}_{4}(0.152 \mathrm{~g}, 4 \mathrm{mmol})$. The reaction mixture was stirred at the same temperature for $2 \mathrm{~h}$ and worked up by treating it sequentially with $\mathrm{H}_{2} \mathrm{O}(0.15 \mathrm{~mL})$, aqueous $\mathrm{NaOH}(15 \%, 0.15 \mathrm{~mL})$, and $\mathrm{H}_{2} \mathrm{O}(0.45 \mathrm{~mL})$ carefully. The resulting mixture was filtered and concentrated. The residue was purified by silica gel flash column chromatography to give enyne 1 in $63 \%$ overall yield. ${ }^{1} \mathrm{H} \mathrm{NMR}\left(500 \mathrm{MHz}, \mathrm{CDCl}_{3}\right) \delta$

\footnotetext{
1 Kende, A. S.; Fludzinski, P.; Hill, J. H.; Swenson, W.; Clardy, J. JACS 1984, 106, 3551-3562
} 
7.41-7.37 (m, 2H), 7.23-7.16 (m, 3H), $4.93(\mathrm{~s}, 1 \mathrm{H}), 4.84(\mathrm{~s}, 1 \mathrm{H}), 3.57$ (d, 1H, J=10.6 Hz), 3.53 (d, $1 \mathrm{H}, J=10.6 \mathrm{~Hz}$ ), 2.43 (d, 1H, J=13.2 Hz), 2.20 (d, 1H, J=13.2 Hz), 1.97 (s, 3H), 1.29 (s, $3 \mathrm{H}) ;{ }^{13} \mathrm{C}$ NMR $\left(125 \mathrm{MHz}, \mathrm{CDCl}_{3}\right) \delta 142.5,131.5,128.2,127.9,123.3,114.7,93.7,83.5,70.6$, 45.8, 37.8, 24.2, 23.6; IR (neat, $\mathrm{cm}^{-1}$ ) 3362 (br), 3077, 2969, 2929, 1653, 1457; MS (EI) Calculated for $\left[\mathrm{C}_{15} \mathrm{H}_{18} \mathrm{O}\right]^{+}:$214.14; Found: 214 .<smiles>C=C(CC(C)C(=O)OCC)c1ccccc1</smiles>
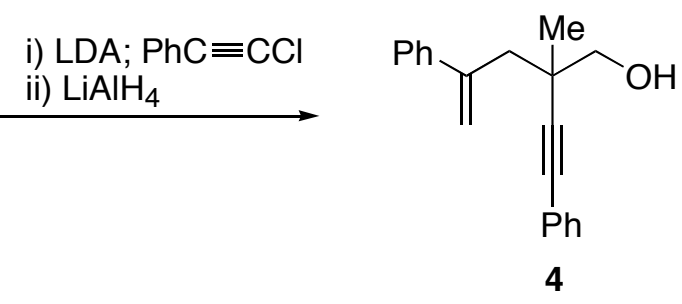

Enyne 4. The title compound was obtained in $27 \%$ yield from ethyl 2-methyl-4-phenylpent-4enoate following the same procedure as for enyne 1. ${ }^{1} \mathrm{H}$ NMR $\left(500 \mathrm{MHz}, \mathrm{CDCl}_{3}\right) \delta 7.45(\mathrm{~d}, 2 \mathrm{H}, J$ $=7.1 \mathrm{~Hz}), 7.30(\mathrm{t}, 2 \mathrm{H}, J=7.1 \mathrm{~Hz}), 7.24-7.19(\mathrm{~m}, 4 \mathrm{H}), 7.15-7.13(\mathrm{~m}, 2 \mathrm{H}), 5.39(\mathrm{~s}, 1 \mathrm{H}), 5.26(\mathrm{~s}$, 1H), 3.50-3.42 (m, 2H), 2.95 (d, 1H, J=13.4 Hz), $2.72(\mathrm{~d}, 1 \mathrm{H}, J=13.4 \mathrm{~Hz}), 1.74$ (t, 1H, J= 7.8 $\mathrm{Hz}), 1.20$ (s, 3H); ${ }^{13} \mathrm{C} \mathrm{NMR}\left(125 \mathrm{MHz}, \mathrm{CDCl}_{3}\right) \delta 145.3,142.7,131.6,128.3,128.0,127.8,127.3$, 126.6, 123.1, 117.6, 93.0, 84.2, 70.0, 42.6, 39.0, 23.7; IR (neat, $\mathrm{cm}^{-1}$ ) 3396 (br), 3080, 3055, 2925, 2852, 1623, 1598, 1491, 1443; MS (APCI) Calculated for $\left[\mathrm{C}_{20} \mathrm{H}_{21} \mathrm{O}\right]^{+}:$277.16; Found: 277.1 .

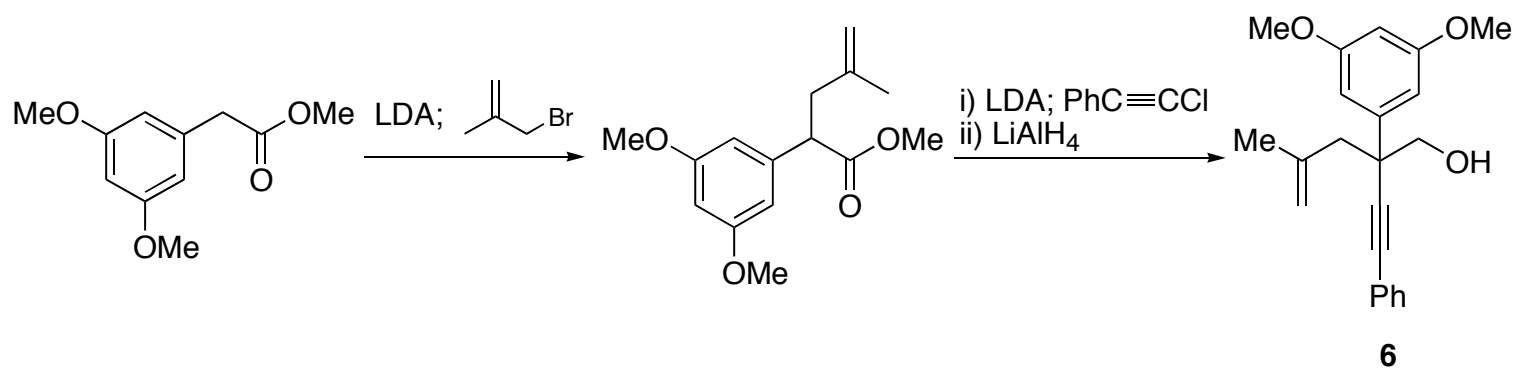

Enyne 6. The title compound was obtained in 31\% yield from methyl 3,5-dimethoxyphenylacetate in three steps through allylation of its enolate (generated by the treatment of LDA) with methallyl bromide followed by the the same procedure as for enyne 1. ${ }^{1} \mathrm{H} \mathrm{NMR}\left(500 \mathrm{MHz}, \mathrm{CDCl}_{3}\right) \delta 7.50$ $7.47(\mathrm{~m}, 2 \mathrm{H}), 7.34-7.32(\mathrm{~m}, 3 \mathrm{H}), 6.78(\mathrm{~d}, 2 \mathrm{H}, J=2.2 \mathrm{~Hz}), 6.39(\mathrm{t}, 1 \mathrm{H}, J=2.2 \mathrm{~Hz}), 4.82(\mathrm{~s}, 1 \mathrm{H})$, $4.75(\mathrm{~s}, 1 \mathrm{H}), 3.84-3.78(\mathrm{~m}, 8 \mathrm{H}), 2.79(\mathrm{~d}, 1 \mathrm{H}, J=13.8 \mathrm{~Hz}), 2.66(\mathrm{~d}, 1 \mathrm{H}, J=13.8 \mathrm{~Hz}), 1.91(\mathrm{t}, 1 \mathrm{H}, J$ $=7.2 \mathrm{~Hz}) ;{ }^{13} \mathrm{C}$ NMR $\left(125 \mathrm{MHz}, \mathrm{CDCl}_{3}\right) \delta 160.7,143.3,141.6,131.6,128.3,128.2,123.1,115.0$, 105.8, 98.5, 91.1, 87.2, 71.1, 55.3, 48.7, 45.5, 24.1; IR (neat, $\mathrm{cm}^{-1}$ ) 3405 (br), 2936, 1596, 1457; MS (APCl) Calculated for $\left[\mathrm{C}_{22} \mathrm{H}_{25} \mathrm{O}\right]^{+}:$337.18; Found: 337.1 . 


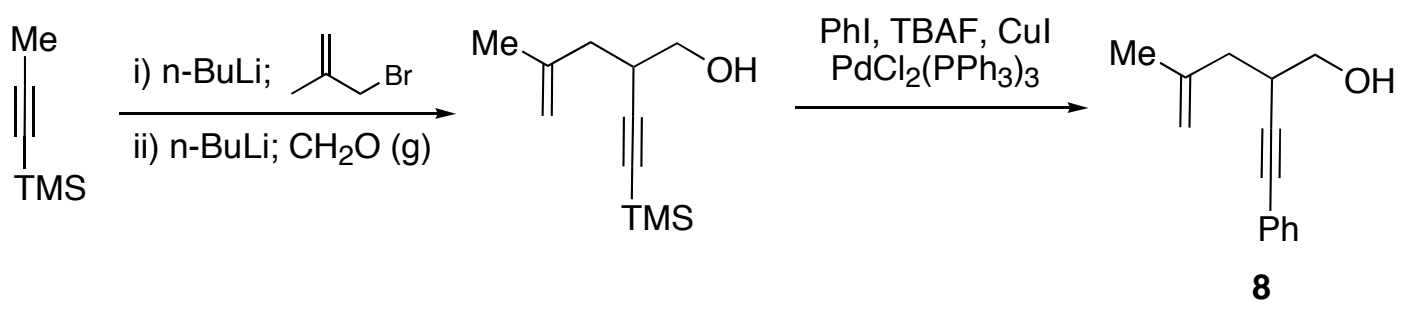

Enyne 8. To a solution of 1-trimethylsilylpropyne $(2.24 \mathrm{~g}, 20 \mathrm{mmol})$ in THF (20 mL) cooled in an ice-water bath was added $\mathrm{n}$-BuLi $(2.5 \mathrm{M}$ in hexanes, $8.4 \mathrm{~mL}, 21 \mathrm{mmol})$. The reaction mixture was stirred for $1 \mathrm{~h}$. Methallyl bromide $(2.88 \mathrm{~g}, 21 \mathrm{mmol})$ was added dropwise and the reaction was allowed to warm to room temperature and stir for $1 \mathrm{~h}$. Then, the reaction mixture was cooled in a dry ice-acetone bath and treated with $\mathrm{n}$-BuLi $(2.5 \mathrm{M}$ in hexanes, $8.4 \mathrm{~mL}, 21 \mathrm{mmol}$ ) and TMEDA $9.34 \mathrm{~mL}, 22 \mathrm{mmol})$. The resulting mixture was warmed to $0^{\circ} \mathrm{C}$ slowly in $1 \mathrm{~h}$ before gaseous formaldehyde (generated by cracking excess paraformaldehyde at $150^{\circ} \mathrm{C}$ ) was bubbled in. The reaction was diluted with $\mathrm{H}_{2} \mathrm{O}$, extracted with $\mathrm{Et}_{2} \mathrm{O}(3 \times 40 \mathrm{~mL})$. The organic layers were combined, washed with $\mathrm{H}_{2} \mathrm{O}$, dried $\left(\mathrm{MgSO}_{4}\right)$, and concentrated. The residue was purified by silica gel flash column chromatography to give TMS-protected enyne as a clear liquid.

The TMS-protected enyne was converted into phenyl alkyne using Sonogashira reaction with TBAF as base. ${ }^{2}$ The enyne $(0.136 \mathrm{~g}, 0.7 \mathrm{mmol})$, phenyl iodide $(0.169 \mathrm{~g}, 0.84 \mathrm{mmol})$, Cul $(0.014$ g, $0.07 \mathrm{mmol})$, and $\mathrm{PdCl}_{2}\left(\mathrm{PPh}_{3}\right)_{2}(0.025 \mathrm{~g}, 0.035 \mathrm{mmol})$ were added to THF $(5 \mathrm{~mL})$ in a roundbottom flask. The resulting mixture was treated with TBAF (1M in THF, $1.5 \mathrm{~mL}$ ) and stirred for $2 \mathrm{~h}$. The reaction mixture was passed through a short celite pad and the filtrate was concentrated. The residue was purified through silica gel flash column chromatography to furnish enyne 8 (0.55 g; $21 \%$ yield from 1-trimethylsilylpropyne). ${ }^{1} \mathrm{H}$ NMR $\left(500 \mathrm{MHz}, \mathrm{CDCl}_{3}\right) \delta$ 7.41-7.39 (m, 2H), 7.30$7.28(\mathrm{~m}, 3 \mathrm{H}), 4.86(\mathrm{~s}, 2 \mathrm{H}), 3.76-3.71(\mathrm{~m}, 1 \mathrm{H}), 3.69-3.64(\mathrm{~m}, 1 \mathrm{H}), 3.02-2.96(\mathrm{~m}, 1 \mathrm{H}), 2.33(\mathrm{~d}, 2 \mathrm{H}, J$ $=7.4 \mathrm{~Hz}), 1.91(\mathrm{br} \mathrm{s}, 1 \mathrm{H}), 1.81(\mathrm{~s}, 3 \mathrm{H}) ;{ }^{13} \mathrm{C} \mathrm{NMR}\left(125 \mathrm{MHz}, \mathrm{CDCl}_{3}\right) \delta 142.6,131.7,128.2,123.2$, 112.8, 89.8, 83.6, 65.2, 39.8, 34.2, 22.4; IR (neat, $\mathrm{cm}^{-1}$ ) 3364 (br), 3077, 2934, 1652, 1490, 1442; MS (EI) Calculated for $\left[\mathrm{C}_{14} \mathrm{H}_{16} \mathrm{O}\right]^{+}: 200.12$; Found: 200.

\footnotetext{
2 Mori, A; Shimada, T; Kondo, T; Sekiguchi, A. Synlett 2001, 649-651
} 


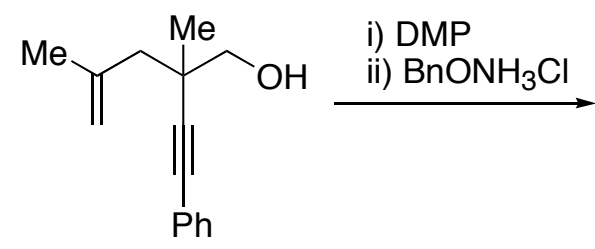

1

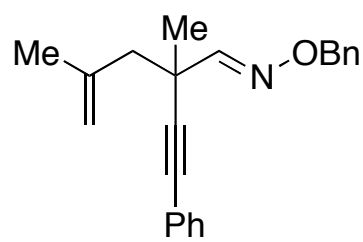

$\mathrm{Ph}$

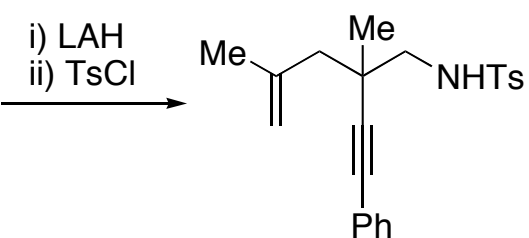

10

Enyne 10. A solution of alcohol $1(0.08 \mathrm{~g}, 0.4 \mathrm{mmol})$ in $\mathrm{CH}_{2} \mathrm{Cl}_{2}(2 \mathrm{~mL})$ cooled in an ice-water bath was treated with Dess-Martin periodinane (15\% solution in $\left.\mathrm{CH}_{2} \mathrm{Cl}, 1.1 \mathrm{~mL}, 0.48 \mathrm{mmol}\right)$. The resulting mixture was warmed to room temperature and stirred for $2 \mathrm{~h}$. The reaction mixture was passed through a short silica pad and the residue obtained after concentration was subjected to the next reaction without further purification.

The crude aldehyde was dissolved in THF $(3 \mathrm{~mL})$, o-Benzyl hydroxylamine hydrogen chloride $(0.128 \mathrm{~g}, 0.8 \mathrm{mmol})$ and $4 \AA$ molecular sieves $(2 \mathrm{~g})$ were added. The mixture was stirred overnight before filtration, concentration, and column purification (hexanes:ethyl acetate $=20: 1$ ) .

To a solution of the o-benzyl oxime obtained above in THF $(2 \mathrm{~mL})$ was added LAH $(0.046 \mathrm{~g}, 1.2$ $\mathrm{mmol})$. The reaction was stirred for $1 \mathrm{~h}$ before being quenched with $\mathrm{MeOH}(1 \mathrm{~mL})$. All solvents were removed under reduced pressure and the residue was partitioned between $\mathrm{CHCl}_{3}(10 \mathrm{~mL})$ and $\mathrm{H}_{2} \mathrm{O}(10 \mathrm{~mL})$. The organic layer was washed with saturated $\mathrm{NH}_{4} \mathrm{Cl}$ and brine, dried $\left(\mathrm{MgSO}_{4}\right)$, and concentrated. The residue was dissolved in $\mathrm{CH}_{2} \mathrm{Cl}_{2}(2 \mathrm{~mL}) . \mathrm{Et}_{3} \mathrm{~N}(0.12 \mathrm{~mL}, 0.8 \mathrm{mmol})$ and $\mathrm{TsCl}(0.096 \mathrm{~g}, 0.5 \mathrm{mmol})$ were added. The reaction was stirred for $2 \mathrm{~h}$ before being evaporated to dryness. The residue was purified through silica gel flash column chromatography to give the sulfonamide $10\left(0.027 \mathrm{~g}, 18 \%\right.$ overall yield). ${ }^{1} \mathrm{H}$ NMR $\left(500 \mathrm{MHz}, \mathrm{CDCl}_{3}\right) \delta 7.75(\mathrm{~d}, 2 \mathrm{H}, J=8.2$ $\mathrm{Hz}$ ), 7.34-7.28 (m, 7H), $4.90(\mathrm{~s}, 1 \mathrm{H}), 4.78(\mathrm{~s}, 1 \mathrm{H}), 4.76(\mathrm{t}, 1 \mathrm{H}, J=6.4 \mathrm{~Hz}), 3.00(\mathrm{dd}, 1 \mathrm{H}, J=6.4$, $11.7 \mathrm{~Hz}$ ), $2.93(\mathrm{dd}, 1 \mathrm{H}, J=6.4,11.7 \mathrm{~Hz}), 2.42(\mathrm{~s}, 3 \mathrm{H}), 2.38(\mathrm{~d}, 1 \mathrm{H}, J=13.3 \mathrm{~Hz}), 2.16(\mathrm{~d}, 1 \mathrm{H}, J=$ $13.3 \mathrm{~Hz}), 1.89(\mathrm{~s}, 3 \mathrm{H}) ;{ }^{13} \mathrm{C}$ NMR $\left(125 \mathrm{MHz}, \mathrm{CDCl}_{3}\right) \delta 143.4,141.8,136.9,131.5,129.7,128.3$, 128.2, 127.0, 122.8, 115.4, 92.7, 83.9, 52.7, 46.9, 35.6, 24.7, 24.1, 21.5; IR (neat, $\mathrm{cm}^{-1}$ ) 3283 (br), $3071,2970,2924,1598,1443$; MS (APCl) Calculated for $\left[\mathrm{C}_{22} \mathrm{H}_{25} \mathrm{NO}_{2} \mathrm{~S}\right]^{+}: 368.17$; Found: 368.1. 


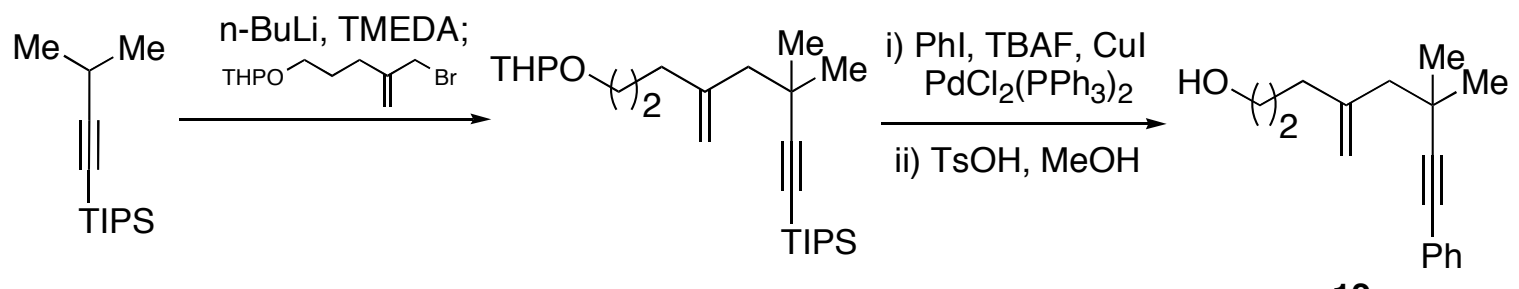

12

Enyne 12. At $-78^{\circ} \mathrm{C}$, to a solution of 3 -methyl-1-triisopropylsilylbutyne ${ }^{3}(0.27 \mathrm{~g}, 1.2 \mathrm{mmol})$ in THF (20 mL) was added $\mathrm{n}$-BuLi $(2.5 \mathrm{M}$ in hexanes, $0.48 \mathrm{~mL})$ and TMEDA $(0.18 \mathrm{~mL}, 1.2 \mathrm{mmol})$. The resulting mixture was warmed to $0^{\circ} \mathrm{C}$ and stirred for $2 \mathrm{~h}$ before being cooled again to $-78^{\circ} \mathrm{C}$. 2-(4bromomethylpent-4-enyloxy)tetrahydropyran ${ }^{4}(0.263 \mathrm{~g}, 1.0 \mathrm{mmol})$ was added and the reaction mixture was allowed to warm to room temperature overnight. Silica gel $(2 \mathrm{~g})$ was added and the slurry was evaporated to dryness before being loaded onto a pre-packed wet column. Elution with hexanes/ethyl acetate gave the tips-protected enyne.

A mixture of the enyne obtained above, THF ( $5 \mathrm{~mL}$ ), and TBAF (1M in THF, $2 \mathrm{~mL}$ ) was heated at a water bath (bath temperature: $50^{\circ} \mathrm{C}$ ) for $1 \mathrm{~h}$. The reaction was cooled to room temperature. Phenyl iodide (0.25 g, $1.2 \mathrm{mmol})$, Cul $(0.023 \mathrm{~g}, 0.12 \mathrm{mmol})$, and $\mathrm{PdCl}_{2}\left(\mathrm{PPh}_{3}\right)_{2}(0.042 \mathrm{~g}, 0.06$ $\mathrm{mmol}$ ) were added and the reaction was stirred for $2 \mathrm{~h}$. The reaction mixture was passed through a short celite pad and the filtrate was concentrated. The residue was dissolved in methanol (5 $\mathrm{mL})$ and $\mathrm{TsOH}(0.005 \mathrm{~g})$ was added. The reaction was stirred for $2 \mathrm{~h}$ at room temperature before being quenched with $\mathrm{Et}_{3} \mathrm{~N}(0.05 \mathrm{~mL})$ and concentrated. The residue was purified through silica gel flash column chromatography to yield enyne 12 (0.11 g, 45\% overall yield). ${ }^{1} \mathrm{H}$ NMR (500 $\left.\mathrm{MHz}, \mathrm{CD}_{2} \mathrm{Cl}_{2}\right) \delta$ 7.36-7.34 (m, 2H), 7.30-7.26 (m, 3H), $4.93(\mathrm{~s}, 1 \mathrm{H}), 4.89(\mathrm{~s}, 1 \mathrm{H}), 3.59(\mathrm{q}, 2 \mathrm{H}, J=$ $6.0 \mathrm{~Hz}), 2.35(\mathrm{t}, 2 \mathrm{H}, J=7.5 \mathrm{~Hz}), 2.27(\mathrm{~s}, 2 \mathrm{H}), 1.78-1.71(\mathrm{~m}, 2 \mathrm{H}), 1.30(\mathrm{~s}, 6 \mathrm{H}) ;{ }^{13} \mathrm{C}$ NMR $(125$ $\left.\mathrm{MHz}, \mathrm{CDCl}_{3}\right) \delta 147.1,131.6,128.6,127.9,124.4,113.6,98.0,80.9,62.9,49.0,33.5,31.5,31.4$, 29.9; IR (neat, cm ${ }^{-1}$ ) 3334 (br), 3080, 2966, 2926, 2867, 2231, 1640, 1598, 1490, 1443; MS (APCI) Calculated for $\left[\mathrm{C}_{17} \mathrm{H}_{23} \mathrm{O}\right]^{+}:$243.17; Found: 243.1.

\footnotetext{
3 it was prepared through the reaction of 3-methylbut-1-ynyl lithium generated from Corey-Fuchs reaction and TIPSOTf

${ }^{4}$ The allyl bromide was obtained by following to a similar procedure: Hitchcock, S. A.; Houldsworth, S. J.; Pattenden, G.; Pryde, D. C.; Thomson, N. M.; Blake, A. J JCS Perk. 1 1998, 3181-3206
} 

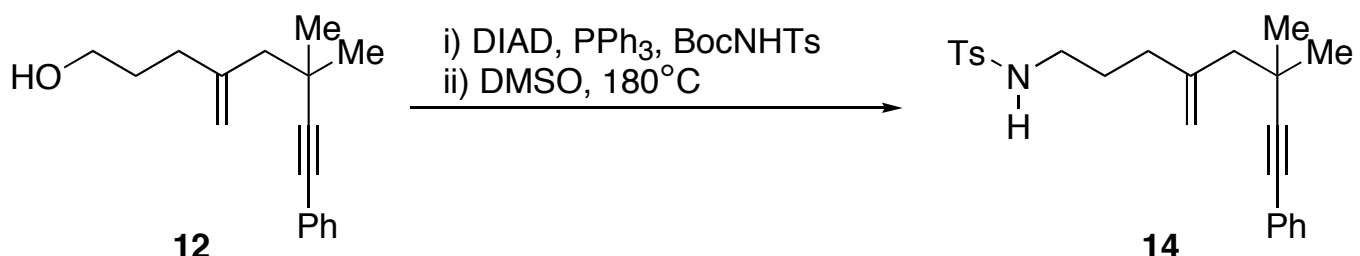

Enyne 14. To a solution of enyne $12(0.031 \mathrm{~g}, 0.128 \mathrm{mmol}), \mathrm{Ph}_{3} \mathrm{P}(0.05 \mathrm{~g}, 0.19 \mathrm{mmol})$, and $\mathrm{N}$ (tert-butoxycarbonyl)-p-toluenesulfonamide $(0.052 \mathrm{~g}, 0.19 \mathrm{mmol})$ in THF was added diisopropyl azodicarboxylate $(37 \mu \mathrm{L}, 0.19 \mathrm{mmol})$ dropwise. The reaction mixture was stirred for $1 \mathrm{~h}$ and then worked up by quenching with $\mathrm{H}_{2} \mathrm{O}$ and extracted with $\mathrm{Et}_{2} \mathrm{O}$. The combined organic layers were washed with $\mathrm{H}_{2} \mathrm{O}$ and brine, dried $\left(\mathrm{MgSO}_{4}\right)$, and concentrated. The residue was purified by silica gel flash column chromatography to yield the Boc-protected sulfonamide.

The sulfonamide obtained above was dissolved in DMSO and the solution was heated in an oil bath (bath temperature: $175-185^{\circ} \mathrm{C}$ ) for $30 \mathrm{~min}$. The reaction was cooled to room temperature and diluted with $\mathrm{H}_{2} \mathrm{O}$. Extraction with EtOAc and column purification yielded enyne 14 in quantitative yield. ${ }^{1} \mathrm{H}$ NMR $\left(500 \mathrm{MHz}, \mathrm{CDCl}_{3}\right) \delta 7.70(\mathrm{~d}, 2 \mathrm{H}, J=8.2 \mathrm{~Hz}), 7.34-7.31(\mathrm{~m}, 2 \mathrm{H}), 7.30-$ 7.25 (m, 5H), 4.84 (s, 1H), 4.83 (s, 1H), 4.45 (bs, 1H), 2.91 (q, 2H, J = $6.7 \mathrm{~Hz}$ ), 2.41 (s, 3H), 2.26 $(\mathrm{t}, 2 \mathrm{H}, J=7.5 \mathrm{~Hz}), 2.17(\mathrm{~s}, 2 \mathrm{H}), 1.69-1.63(\mathrm{~m}, 2 \mathrm{H}), 1.26(\mathrm{~s}, 6 \mathrm{H}) ;{ }^{13} \mathrm{C} \mathrm{NMR}\left(125 \mathrm{MHz}, \mathrm{CDCl}_{3}\right) \delta$ 145.5, 143.3, 137.0, 131.3, 129.6, 128.2, 127.5, 127.0, 123.8, 114.3, 97.4, 80.7, 48.5, 42.9, 33.7, 31.0, 29.7, 27.7, 21.5; IR (neat, $\mathrm{cm}^{-1}$ ) 3282 (br), 3065, 2967, 2925, 2867, 1640, 1598, 1490, 1443; MS (APCI) Calculated for $\left[\mathrm{C}_{24} \mathrm{H}_{30} \mathrm{NO}_{2} \mathrm{~S}\right]^{+}:$396.57; Found: 396.1 .

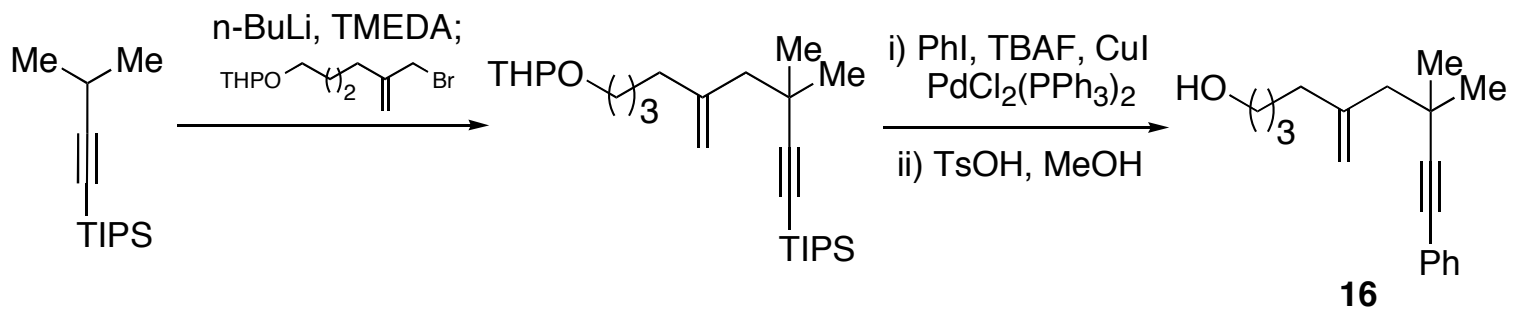

Enyne 16. The title compound was obtained in $31 \%$ overall yield by using the same procedure as for enyne 12. ${ }^{1} \mathrm{H}$ NMR $\left(500 \mathrm{MHz}, \mathrm{CDCl}_{3}\right) \delta$ 7.29-7.27 (m, 2H), 7.21-7.15 (m, 3H), $4.83(\mathrm{~s}, 1 \mathrm{H})$, $4.79(\mathrm{~s}, 1 \mathrm{H}), 3.53(\mathrm{t}, 2 \mathrm{H}, J=5.8 \mathrm{~Hz}), 2.24(\mathrm{t}, 2 \mathrm{H}, J=6.4 \mathrm{~Hz}), 2.17(\mathrm{~s}, 2 \mathrm{H}), 1.50-1.46(\mathrm{~m}, 4 \mathrm{H}), 1.22$ (s, 6H); ${ }^{13} \mathrm{C}$ NMR $\left(125 \mathrm{MHz}, \mathrm{CDCl}_{3}\right) \delta 146.7,131.3,128.1,127.4,124.0,113.4,96.7,80.5,62.8$, 48.8, 36.5, 32.4, 31.0, 29.7, 24.1; IR (neat, $\mathrm{cm}^{-1}$ ) 3339 (br), 3080, 2966, 2933, 2865, 1640, 1598, 1443; MS (APCI) Calculated for $\left[\mathrm{C}_{18} \mathrm{H}_{25} \mathrm{O}\right]^{+}:$257.15; Found: 257.1. 


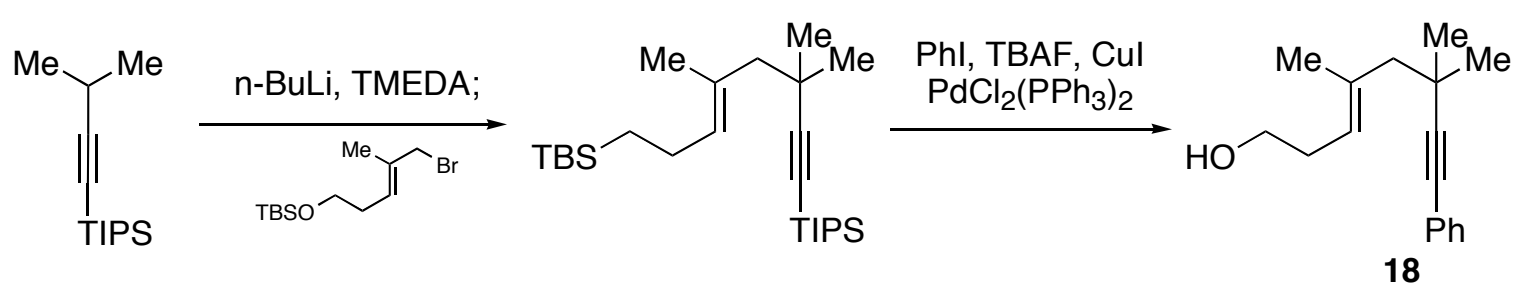

Enyne 18. At $-78^{\circ} \mathrm{C}$, to a solution of 3-methyl-1-triisopropylsilylbutyne ${ }^{3}(0.27 \mathrm{~g}, 1.2 \mathrm{mmol})$ in THF (20 mL) was added $\mathrm{n}$-BuLi $(2.5 \mathrm{M}$ in hexanes, $0.48 \mathrm{~mL})$ and TMEDA $(0.18 \mathrm{~mL}, 1.2 \mathrm{mmol})$. The resulting mixture was warmed to $0^{\circ} \mathrm{C}$ and stirred for $2 \mathrm{~h}$ before being cooled again to $-78^{\circ} \mathrm{C}$. (5Bromo-4-methylpent-3-enyloxy)-tert-butyldimethylsilane ${ }^{5}(0.293 \mathrm{~g}, 1.0 \mathrm{mmol})$ was added and the reaction mixture was allowed to warm to room temperature overnight. Silica gel $(2 \mathrm{~g})$ was added and the slurry was evaporated to dryness before being loaded onto a pre-packed wet column. Elution with hexanes/ethyl acetate gave the tips-protected enyne.

A mixture of the enyne obtained above, THF $(5 \mathrm{~mL})$, and TBAF (1M in THF, $3 \mathrm{~mL}$ ) was heated at a water bath (bath temperature: $50^{\circ} \mathrm{C}$ ) for $1 \mathrm{~h}$. The reaction was cooled to room temperature. Phenyl iodide (0.25 g, $1.2 \mathrm{mmol})$, Cul $(0.023 \mathrm{~g}, 0.12 \mathrm{mmol})$, and $\mathrm{PdCl}_{2}\left(\mathrm{PPh}_{3}\right)_{2}(0.042 \mathrm{~g}, 0.06$ $\mathrm{mmol}$ ) were added and the reaction was stirred for $2 \mathrm{~h}$. The reaction mixture was passed through a short celite pad and the filtrate was concentrated. The residue was purified through silica gel flash column chromatography to yield enyne 18 in $55 \%$ overall yield as a mixture of geometric isomers $(E / Z=9 / 1)$. For the $E$-isomer: ${ }^{1} \mathrm{H}$ NMR $\left(500 \mathrm{MHz}, \mathrm{CDCl}_{3}\right) \delta$ 7.31-7.28 $(\mathrm{m}, 2 \mathrm{H}), 7.21-7.19$ (m, 3H), 5.19 (t, 1H, J=7.2 Hz), 3.56 (t, 2H, J=6.7 Hz), 2.27 (q, 2H, J = 6.7 Hz), 2.16 (s, 2H), $1.79(\mathrm{~s}, 3 \mathrm{H}), 1.21(\mathrm{~s}, 6 \mathrm{H}) ;{ }^{13} \mathrm{C}$ NMR $\left(125 \mathrm{MHz}, \mathrm{CDCl}_{3}\right)$ E-isomer: $\delta 136.3,131.3,128.2,127.5$, 124.4, 123.9, 97.7, 80.9, 62.3, 52.8, 31.68, 31.66, 29.8, 18.3; IR (neat, $\mathrm{cm}^{-1}$ ) 3337, 3054, 2966, 2922, 2228, 1579, 1490, 1443, 1360; MS (APCl) Calculated for $\left[\mathrm{C}_{17} \mathrm{H}_{23} \mathrm{O}\right]^{+}:$243.17; Found: 243.1 .
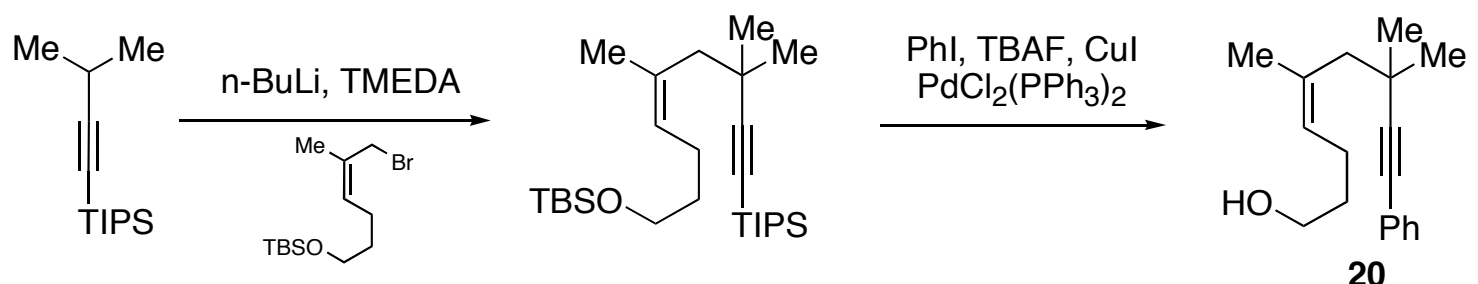

Enyne 20. The title compound was obtained in $60 \%$ overall yield as the $Z$-isomer only by using the same procedure as for enyne 18. ${ }^{1} \mathrm{H}$ NMR $\left(500 \mathrm{MHz}, \mathrm{CDCl}_{3}\right) \delta 7.37-7.35(\mathrm{~m}, 2 \mathrm{H}), 7.28-7.22$ (m, 3H), 5.31 (t, 1H, J=7.0 Hz), 3.62 (q, 2H, J=6.0 Hz), 2.30 (s, 2H), 2.11 (q, 2H, J = 7.2 Hz), $1.94(\mathrm{~s}, 3 \mathrm{H}), 1.60(\mathrm{p}, 2 \mathrm{H}, J=6.8 \mathrm{~Hz}), 1.41(\mathrm{t}, 1 \mathrm{H}, J=5.3 \mathrm{~Hz}), 1.30(\mathrm{~s}, 6 \mathrm{H}) ;{ }^{13} \mathrm{C}$ NMR $(125 \mathrm{MHz}$,

\footnotetext{
${ }^{5}$ As a mixture of isomers $(E / Z=9 / 1)$.
} 
$\left.\mathrm{CDCl}_{3}\right): \delta 133.4,131.3,128.11,128.06,127.4,124.0,98.2,80.5,62.6,44.5,32.8,31.8,30.0$, 25.7, 24.9; IR (neat, $\mathrm{cm}^{-1}$ ) 3339, 2964, 2933, 2867, 1598, 1491, 1443, 1479, 1360; MS (APCI) Calculated for $\left[\mathrm{C}_{18} \mathrm{H}_{25} \mathrm{O}\right]^{+}:$257.19; Found: 257.1.



Enyne 22. The title compound was obtained from enyne 18 in $52 \%$ yield by using the same procedure as for enyne 14. ${ }^{1} \mathrm{H}$ NMR $\left(500 \mathrm{MHz}, \mathrm{CDCl}_{3}\right) \delta 7.70(\mathrm{~d}, 2 \mathrm{H}, J=8.1 \mathrm{~Hz}), 7.35-7.33(\mathrm{~m}$, 2H), 7.27-7.25 (m, 5H), 5.07 (t, 1H, J=7.2 Hz), 4.20 (t, $1 \mathrm{H}, J=6.5 \mathrm{~Hz}), 2.97$ (q, 2H, J = $6.5 \mathrm{~Hz}$ ), $2.41(\mathrm{~s}, 3 \mathrm{H}), 2.21(\mathrm{dt}, 2 \mathrm{H}, J=6.5,7.2 \mathrm{~Hz}), 2.16(\mathrm{~s}, 2 \mathrm{H}), 1.77(\mathrm{~s}, 3 \mathrm{H}), 1.25(\mathrm{~s}, 6 \mathrm{H}) ;{ }^{13} \mathrm{C}$ NMR $(125$ $\left.\mathrm{MHz}, \mathrm{CDCl}_{3}\right) \delta 143.3,137.0,136.7,131.3,129.6,128.2,127.5,127.0,124.0,123.9,97.6,81.0$, 52.6, 42.8, 31.5, 30.0, 29.7, 28.3, 21.5, 18.2; IR (neat, $\mathrm{cm}^{-1}$ ) 3290 (br), 2969, 2925, 1684, 1598, 1491, 1442; MS (APCI) Calculated for $\left[\mathrm{C}_{24} \mathrm{H}_{30} \mathrm{NO}_{2} \mathrm{~S}\right]^{+}: 396.57$; Found: 396.1 .

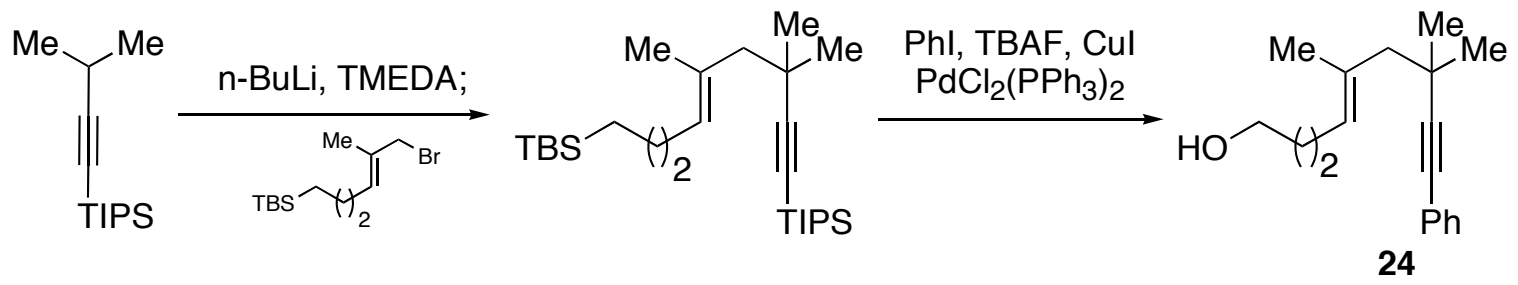

Enyne 24. The title compound was obtained in $45 \%$ overall yield as the $E$-isomer only by using the same procedure as for enyne 18. ${ }^{1} \mathrm{H}$ NMR $\left(500 \mathrm{MHz}, \mathrm{CDCl}_{3}\right) \delta$ 7.37-7.35 (m, 2H), 7.28-7.24 (m, 3H), 5.25 (t, 1H, J=7.1 Hz), 3.64 (t, 2H, J=6.5 Hz), 2.20 (s, 2H), 2.12 (q, 2H, J = 7.1 Hz), $1.83(\mathrm{~s}, 3 \mathrm{H}), 1.63(\mathrm{p}, 2 \mathrm{H}, J=7.1 \mathrm{~Hz}), 1.27(\mathrm{~s}, 6 \mathrm{H}) ;{ }^{13} \mathrm{C} \mathrm{NMR}\left(125 \mathrm{MHz}, \mathrm{CDCl}_{3}\right): \delta 133.3,131.3$, 128.3, 128.1, 127.3, 124.1, 98.0, 80.7, 62.6, 52.8, 32.6, 31.5, 29.7, 24.4, 18.0; IR (neat, $\mathrm{cm}^{-1}$ ) $3330,2965,2927,2229,1598,1491,1443,1380$; MS (APCl) Calculated for $\left[\mathrm{C}_{18} \mathrm{H}_{25} \mathrm{O}\right]^{+}: 257.19$; Found: 257.1.

\section{Gold-catalyzed Assembly of Heterobicyclic Systems}

General Procedure A: To a solution of an alcohol (or sulfonamide 10) in acetonitrile $(0.05 \mathrm{M})$ at room temperature was added a solution of $\mathrm{AuCl}_{3}$ in acetonitrile $(5 \mathrm{~mol} \%, 7.2 \mu \mathrm{M} ; 10 \mathrm{~mol} \%$ in the 
cases of alcohols 18 and 20). The reaction mixture was stirred for $1 \mathrm{~h}$ and then treated with $\mathrm{Et}_{3} \mathrm{~N}$ $(0.05 \mathrm{~mL})$. Concentration and silica gel column chromatography yielded the cyclized product.

General Procedure B: A solution of $\left[\mathrm{Au}\left(\mathrm{PPh}_{3}\right)\right]^{+} \mathrm{ClO}_{4}^{-}$in $\mathrm{CH}_{2} \mathrm{Cl}_{2}$ was prepared as following: $\mathrm{AuCl}\left(\mathrm{PPh}_{3}\right)(70 \mathrm{mg}, 0.2 \mathrm{mmol})$ was dissolved in $\mathrm{CH}_{2} \mathrm{Cl}_{2}(10 \mathrm{~mL})$. The solution was treated with $\mathrm{AgClO}_{4}(42 \mathrm{mg}, 0.2 \mathrm{mmol}$ ) and stirred for $1 \mathrm{~h}$. $\mathrm{AgCl}$ precipitation formed gradually and the supernatant was used for the following reactions.

A solution of the alcohol in $\mathrm{CH}_{2} \mathrm{Cl}_{2}(0.05 \mathrm{M})$ at room temperature was treated with the $\left[\mathrm{Au}\left(\mathrm{PPh}_{3}\right)\right]^{+}$ $\mathrm{ClO}_{4}^{-}$solution ( $5 \mathrm{~mol} \%$ ) generated previously. The reaction mixture was stirred for $1 \mathrm{~h}$ and then treated with $\mathrm{Et}_{3} \mathrm{~N}(0.05 \mathrm{~mL})$. Concentration and silica gel column chromatography yielded the cyclized product.

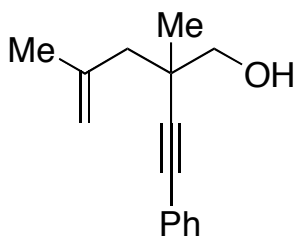

1

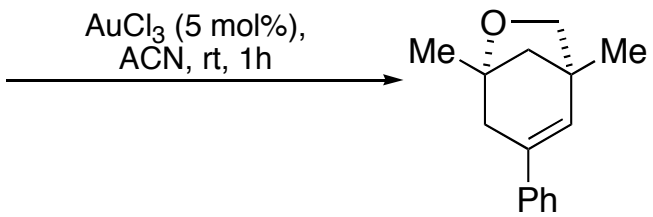

2

Alkene 2 was obtained in $89 \%$ yield according to the general procedure A. ${ }^{1} \mathrm{H}$ NMR $(500 \mathrm{MHz}$, $\left.\mathrm{CDCl}_{3}\right) \delta 7.40(\mathrm{~d}, 2 \mathrm{H}, J=7.6 \mathrm{~Hz}), 7.31(\mathrm{t}, 2 \mathrm{H}, J=7.6 \mathrm{~Hz}), 7.23(\mathrm{t}, 1 \mathrm{H}, J=7.6 \mathrm{~Hz}), 6.23(\mathrm{~s}, 1 \mathrm{H})$, $3.84(\mathrm{~d}, 1 \mathrm{H}, J=6.8 \mathrm{~Hz}), 3.64(\mathrm{~d}, 1 \mathrm{H}, J=6.8 \mathrm{~Hz}), 1.78(\mathrm{~d}, 1 \mathrm{H}, J=10.6 \mathrm{~Hz}), 1.72(\mathrm{~d}, 1 \mathrm{H}, J=10.6$ $\mathrm{Hz}), 1.48(\mathrm{~s}, 3 \mathrm{H}), 1.27$ (s, 3H); ${ }^{13} \mathrm{C}$ NMR $\left(125 \mathrm{MHz}, \mathrm{CDCl}_{3}\right) \delta 140.1,135.2,133.1,128.2,127.1$, 124.9, 82.3, 81.1, 47.5, 43.6, 42.3, 26.0, 20.2; IR (neat, $\mathrm{cm}^{-1}$ ) 3348, 3024, 2961, 2925, 2869, 1652, 1456, 1376; MS (EI) Calculated for $\left[\mathrm{C}_{15} \mathrm{H}_{19} \mathrm{O}\right]^{+}:$214.15; Found: 214.

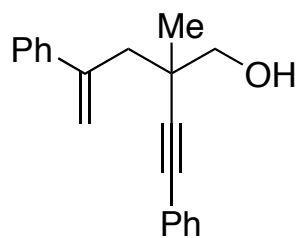

4

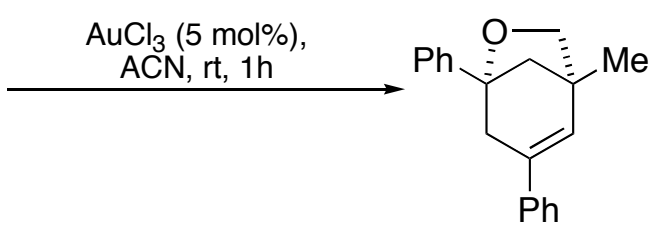

5

Alkene 5 was obtained in $90 \%$ yield according to the general procedure A. ${ }^{1} \mathrm{H}$ NMR $(500 \mathrm{MHz}$, $\left.\mathrm{CDCl}_{3}\right) \delta 7.52(\mathrm{~d}, 2 \mathrm{H}, J=7.5 \mathrm{~Hz}), 7.43(\mathrm{~d}, 2 \mathrm{H}, J=7.5 \mathrm{~Hz}), 7.39(\mathrm{t}, 2 \mathrm{H}, J=7.5 \mathrm{~Hz}), 7.33-7.28(\mathrm{~m}$, 
3H), $7.25(\mathrm{t}, 1 \mathrm{H}, J=7.5 \mathrm{~Hz}), 6.33(\mathrm{~s}, 1 \mathrm{H}), 4.06(\mathrm{~d}, 1 \mathrm{H}, J=6.8 \mathrm{~Hz}), 3.77(\mathrm{~d}, 1 \mathrm{H}, J=6.8 \mathrm{~Hz}), 2.92$ (d, $1 \mathrm{H}, J=6.8 \mathrm{~Hz}$ ), $2.85(\mathrm{~d}, 1 \mathrm{H}, J=12.3 \mathrm{~Hz}), 2.27(\mathrm{~d}, 1 \mathrm{H}, J=10.6 \mathrm{~Hz}), 2.04(\mathrm{~d}, 1 \mathrm{H}, J=10.6 \mathrm{~Hz})$, 1.33 (s, 3H); ${ }^{13} \mathrm{C}$ NMR (125 MHz, acetone-d6) $\delta$ 147.2, 141.0, 135.9, 133.9, 129.3, 129.1, 128.1, 127.8, 126.0, 125.8, 85.3, 83.1, 47.9, 45.5, 43.1, 19.9; IR (neat, $\mathrm{cm}^{-1}$ ) 3057, 3026, 2958, 2925, 2852, 1600, 1495, 1447, 1357; MS (APCI) Calculated for $\left[\mathrm{C}_{20} \mathrm{H}_{21} \mathrm{O}\right]^{+}: 277.16$; Found: 277.1.<smiles>C=C(C)CC(C#CCO)(CO)c1cc(OC)cc(OC)c1</smiles>

6

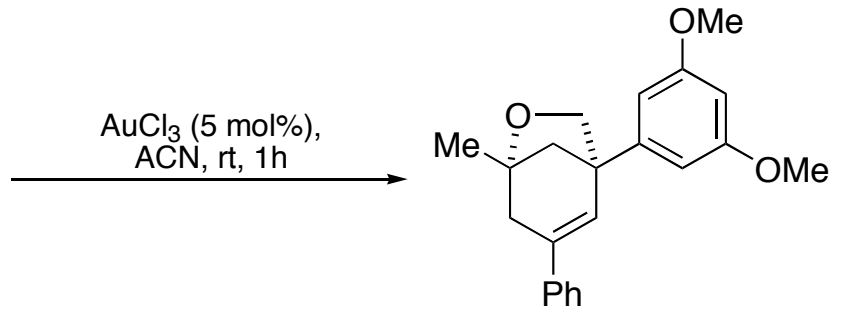

7

Alkene 7 was obtained in $92 \%$ yield according to the general procedure A. ${ }^{1} \mathrm{H}$ NMR $(500 \mathrm{MHz}$, $\left.\mathrm{CDCl}_{3}\right) \delta 7.41(\mathrm{~d}, 2 \mathrm{H}, J=7.8 \mathrm{~Hz}), 7.30(\mathrm{t}, 2 \mathrm{H}, J=7.8 \mathrm{~Hz}), 7.24(\mathrm{t}, 1 \mathrm{H}, J=7.8 \mathrm{~Hz}), 6.453(\mathrm{~s}, 2 \mathrm{H})$, $6.448(\mathrm{~s}, 1 \mathrm{H}), 6.40(\mathrm{~s}, 1 \mathrm{H}), 4.27(\mathrm{~d}, 1 \mathrm{H}, J=6.7 \mathrm{~Hz}), 4.03(\mathrm{~d}, 1 \mathrm{H}, J=6.7 \mathrm{~Hz}), 3.81(\mathrm{~s}, 6 \mathrm{H}), 2.74(\mathrm{~d}$, $1 \mathrm{H}, J=17.3 \mathrm{~Hz}$ ), $2.66(\mathrm{~d}, 1 \mathrm{H}, J=17.3 \mathrm{~Hz}), 2.21(\mathrm{~d}, 1 \mathrm{H}, J=10.4 \mathrm{~Hz}), 2.15(\mathrm{~d}, 1 \mathrm{H}, J=10.4 \mathrm{~Hz})$, $1.56(\mathrm{~s}, 3 \mathrm{H}) ;{ }^{13} \mathrm{C}$ NMR $\left(125 \mathrm{MHz}, \mathrm{CDCl}_{3}\right) \delta 161.0,145.7,139.8,135.1^{\prime}, 133.0,128.3,127.3$, 125.1, 104.7, 98.2, 80.9, 80.8, 55.3, 51.0, 45.1, 43.5, 229.7, 26.1; IR (neat, $\mathrm{cm}^{-1}$ ) 2962, 2928, 2855, 1597, 1457, 1424, 1358; MS (APCl) Calculated for $\left[\mathrm{C}_{22} \mathrm{H}_{25} \mathrm{O}_{3}\right]^{+}: 337.18$; Found: 337.1.

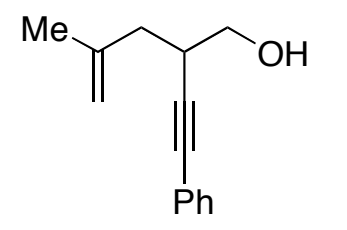

8

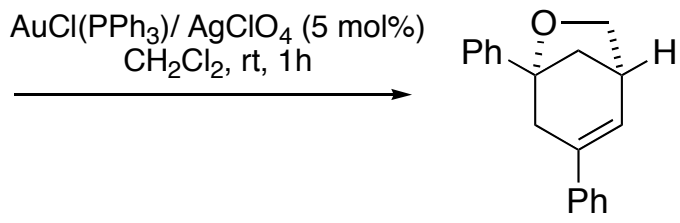

9

Alkene 9 was obtained in $89 \%$ yield according to the general procedure B. ${ }^{1} \mathrm{H}$ NMR $(500 \mathrm{MHz}$, $\left.\mathrm{CDCl}_{3}\right) \delta 7.39(\mathrm{~d}, 2 \mathrm{H}, J=7.8 \mathrm{~Hz}), 7.31$ (dd, $\left.2 \mathrm{H}, J=7.2,7.8 \mathrm{~Hz}\right), 6.50(\mathrm{~d}, 1 \mathrm{H}, J=6.8 \mathrm{~Hz}$ ), 3.96$3.92(\mathrm{~m}, 2 \mathrm{H}), 2.87$ (br s, 1H), 2.64-2.56 (m, 2H), $1.86(\mathrm{~d}, 1 \mathrm{H}, J=5.5 \mathrm{~Hz}), 1.82$ (dd, $1 \mathrm{H}, J=3.4$, $5.5 \mathrm{~Hz}), 1.49(\mathrm{~s}, 3 \mathrm{H}) ;{ }^{13} \mathrm{C}$ NMR $\left(125 \mathrm{MHz}, \mathrm{CDCl}_{3}\right) \delta$ 140.2, 135.5, 128.2, 128.1, 127.0, 124.9, 79.9, 76.8, 44.0, 40.3, 37.6, 25.8; IR (neat, $\mathrm{cm}^{-1}$ ) 3054, 3031, 2965, 2925, 2858, 1598, 1494, 1446, 1376; MS (EI) Calculated for $\left[\mathrm{C}_{14} \mathrm{H}_{16} \mathrm{O}\right]^{+}: 200.12$; Found: 200. 


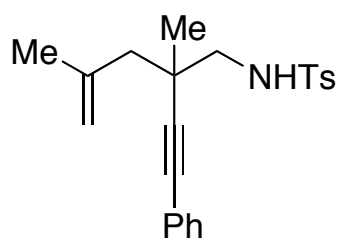

10

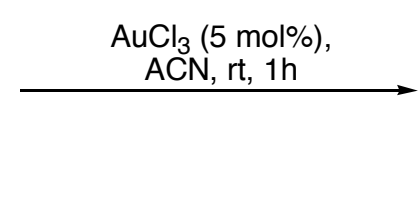

ACN, rt, $1 \mathrm{~h}$

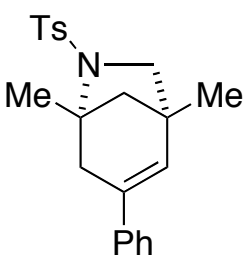

11

Alkene 11 was obtained in $82 \%$ yield according to the general procedure A. ${ }^{1} \mathrm{H}$ NMR $(500 \mathrm{MHz}$, $\left.\mathrm{CDCl}_{3}\right) \delta 7.63(\mathrm{~d}, 2 \mathrm{H}, J=8.3 \mathrm{~Hz}), 7.26-7.20(\mathrm{~m}, 3 \mathrm{H}), 7.14-7.10(\mathrm{~m}, 2 \mathrm{H}), 7.03(\mathrm{~d}, 2 \mathrm{H}, J=8.3 \mathrm{~Hz})$, $5.96(\mathrm{~s}, 1 \mathrm{H}), 3.56(\mathrm{~d}, 1 \mathrm{H}, J=8.7 \mathrm{~Hz}), 3.25(\mathrm{~d}, 1 \mathrm{H}, J=8.7 \mathrm{~Hz}), 2.98(\mathrm{~d}, 1 \mathrm{H}, J=17.5 \mathrm{~Hz}), 2.46$ (d, $1 \mathrm{H}, J=17.5 \mathrm{~Hz}), 2.25(\mathrm{~s}, 3 \mathrm{H}), 1.84(\mathrm{~d}, 1 \mathrm{H}, J=10.7 \mathrm{~Hz}), 1.73(\mathrm{~d}, 1 \mathrm{H}, J=10.7 \mathrm{~Hz}), 1.71(\mathrm{~s}, 3 \mathrm{H})$, 1.20 (s, 3H); ${ }^{13} \mathrm{C} \mathrm{NMR}\left(125 \mathrm{MHz}, \mathrm{CDCl}_{3}\right) \delta 142.4 .139 .4,138.6,135.3,131.2,129.2,128.0,127.2$, 126.9, 124.9, 66.3, 63.7, 50.8, 41.3, 38.6, 29.7, 26.2, 21.3; IR (neat, $\mathrm{cm}^{-1}$ ) 3026, 2958, 2926, 2871, 1598, 1446, 1337; MS (APCl) Calculated for $\left[\mathrm{C}_{22} \mathrm{H}_{25} \mathrm{NO}_{2} \mathrm{~S}\right]^{+}:$368.17; Found: 368.1.

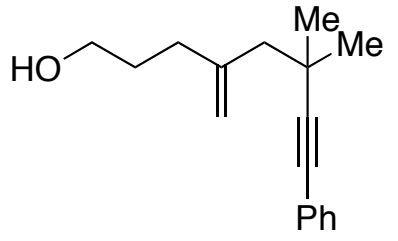

12

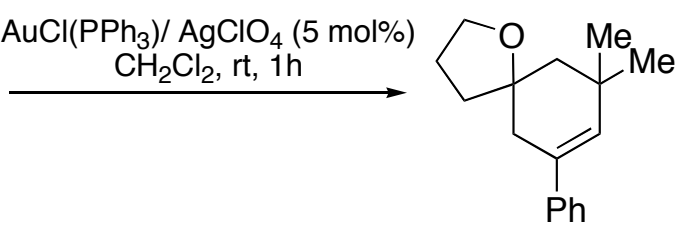

13

Alkene 13 was obtained in $86 \%$ yield according to the general procedure B. ${ }^{1} \mathrm{H}$ NMR $(500 \mathrm{MHz}$, $\mathrm{CDCl}_{3}$ ) $\delta 7.39$ (d, $2 \mathrm{H}, J=8.0 \mathrm{~Hz}$ ), 7.31 (dd, $\left.2 \mathrm{H}, J=7.4,8.0 \mathrm{~Hz}\right), 7.22(\mathrm{t}, 1 \mathrm{H}, J=7.4 \mathrm{~Hz}), 5.86(\mathrm{~s}$, $1 \mathrm{H})$, 3.92-3.82 (m, 2H), $2.48(\mathrm{~s}, 2 \mathrm{H}), 1.98(\mathrm{p}, 2 \mathrm{H}, J=7.3 \mathrm{~Hz}), 1.88-1.83(\mathrm{~m}, 1 \mathrm{H}), 1.82(\mathrm{~d}, 1 \mathrm{H}, J=$ $13.5 \mathrm{~Hz}), 1.78-1.73(\mathrm{~m}, 1 \mathrm{H}), 1.56(\mathrm{~d}, 1 \mathrm{H}, J=13.5 \mathrm{~Hz}), 1.19(\mathrm{~s}, 3 \mathrm{H}), 1.11(\mathrm{~s}, 3 \mathrm{H}) ;{ }^{13} \mathrm{C}$ NMR $(125$ $\left.\mathrm{MHz}, \mathrm{CDCl}_{3}\right) \delta 142.1,134.4,131.8,128.1,126.6,125.3,81.8,66.4,46.0,38.7,37.6,34.1,31.2$, 30.6, 25.5; IR $\left(\right.$ neat, $\left.\mathrm{cm}^{-1}\right)$ 2951. 1598. 1493. 1358; MS (EI) Calculated for $\left[\mathrm{C}_{17} \mathrm{H}_{22} \mathrm{O}\right]^{+}: 242.17$; Found: 242.

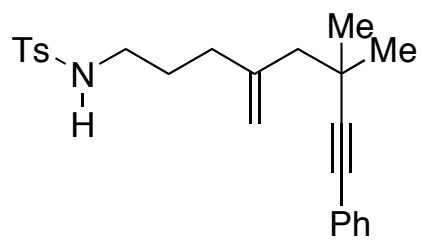

14

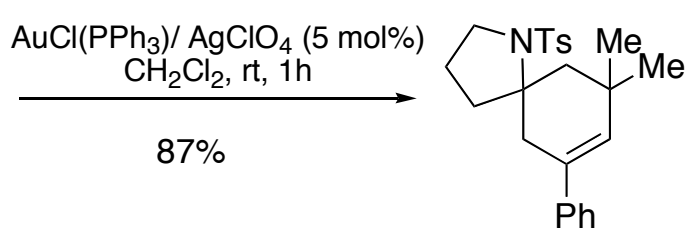

15

Alkene 15 was obtained in $87 \%$ yield according to the general procedure B. ${ }^{1} \mathrm{H}$ NMR $(500 \mathrm{MHz}$, $\left.\mathrm{CDCl}_{3}\right) \delta 7.77(\mathrm{~d}, 2 \mathrm{H}, J=8.1 \mathrm{~Hz}), 7.36-7.22(\mathrm{~m}, 7 \mathrm{H}), 5.75(\mathrm{~s}, 1 \mathrm{H}), 3.67-3.63(\mathrm{~m}, 1 \mathrm{H}), 2.77(\mathrm{~d}, 1 \mathrm{H}$, $J=12.9 \mathrm{~Hz}), 2.44(\mathrm{~s}, 3 \mathrm{H}), 2.36(\mathrm{~d}, 1 \mathrm{H}, J=16.2 \mathrm{~Hz}), 2.02-1.98(\mathrm{~m}, 1 \mathrm{H}), 1.86-1.74(\mathrm{~m}, 3 \mathrm{H}), 1.46$ 
(d, $1 \mathrm{H}, J=12.9 \mathrm{~Hz}), 1.14$ (s, 3H), 1.40 (s, 3H); ${ }^{13} \mathrm{C}$ NMR (125 MHz, $\left.\mathrm{CDCl}_{3}\right) \delta$ 142.6, 142.0, 139.2, 133.6, 132.8, 129.4, 128.2, 127.0, 126.8, 125.3, 69.0, 48.9, 45.5, 39.3, 38.4, 34.8, 33.3, 29.4, 23.1, 21.5; IR (neat, $\mathrm{cm}^{-1}$ ) 2956, 2925, 2867, 1598, 1494, 1446; MS (APCI) Calculated for $\left[\mathrm{C}_{24} \mathrm{H}_{30} \mathrm{NO}_{2} \mathrm{~S}\right]^{+}:$396.57; Found: 396.1 .

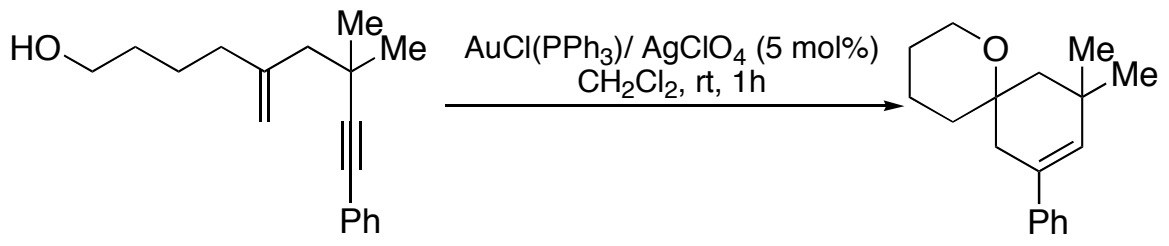

16

17

Alkene 17 was obtained in $96 \%$ yield according to the general procedure B. ${ }^{1} \mathrm{H}$ NMR $(500 \mathrm{MHz}$, $\left.\mathrm{CDCl}_{3}\right) \delta 7.41$ (d, 2H, J=7.6 Hz), 7.31 (t, 2H, J=7.6 Hz), 7.22 (t, 2H, J=7.6 Hz), $5.83(\mathrm{~s}, 1 \mathrm{H})$, 3.72-3.64 (m, 2H), 2.59 (d, 1H, J = 16.9 Hz), $2.42(\mathrm{~d}, 1 \mathrm{H}, J=16.9 \mathrm{~Hz}), 2.07(\mathrm{~d}, 1 \mathrm{H}, J=14.1 \mathrm{~Hz})$, 1.71-1.52 (m, 6H), $1.38(\mathrm{~d}, 1 \mathrm{H}, J=14.1 \mathrm{~Hz}), 1.19(\mathrm{~s}, 3 \mathrm{H}), 1.09(\mathrm{~s}, 3 \mathrm{H}) ;{ }^{13} \mathrm{C}$ NMR $(125 \mathrm{MHz}$, $\left.\mathrm{CDCl}_{3}\right) \delta 142.3,134.0,130.8,128.1,126.6,125.4,72.5,61.4,42.5,38.4,36.5,33.2,31.9,30.0$, 26.0, 19.4; IR (neat, $\mathrm{cm}^{-1}$ ) 2928, 2860, 1598, 1493, 1443; MS (EI) Calculated for $\left[\mathrm{C}_{18} \mathrm{H}_{24} \mathrm{O}\right]^{+}$: 256.18; Found: 256.

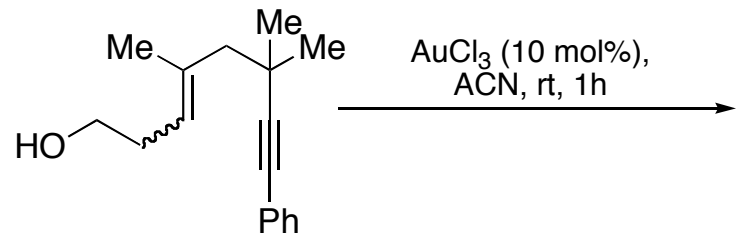

$E / Z: 9 / 1$

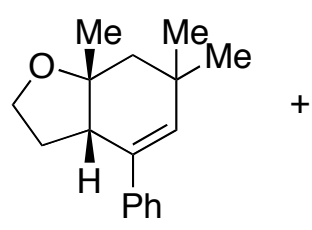

1

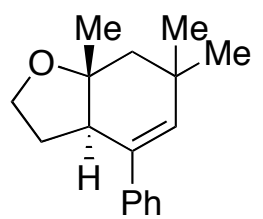

9

18

19

Starting from enyne $18(E / Z=9 / 1), A$ 9/1 mixture of 19 and its cis-fused isomer was formed in a combined yield of $86 \%$ according to the general procedure $E\left(10 \mathrm{~mol} \% \mathrm{AuCl}_{3}\right)$. The diastereomers were separated using silica gel flash column chromatography. 


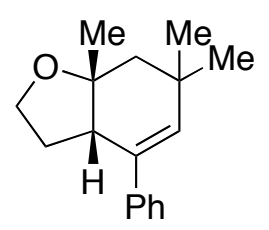

5,6-cis diastereomer: ${ }^{1} \mathrm{H}$ NMR $\left(500 \mathrm{MHz}, \mathrm{CDCl}_{3}\right) \delta 7.37(\mathrm{~d}, 2 \mathrm{H}, J=7.5 \mathrm{~Hz}), 7.32(\mathrm{dd}, 2 \mathrm{H}, J=7.1$, $7.5 \mathrm{~Hz}$ ), 7.24 (t, $1 \mathrm{H}, J=7.1 \mathrm{~Hz}$ ), $5.82(\mathrm{~s}, 1 \mathrm{H}), 3.88-3.75(\mathrm{~m}, 2 \mathrm{H}), 2.94(\mathrm{dd}, 1 \mathrm{H}, J=4.9,7.6 \mathrm{~Hz})$, 2.33-2.26 (m, $1 \mathrm{H}), 1.87(\mathrm{~d}, 1 \mathrm{H}, J=14.0 \mathrm{~Hz}), 1.66-1.60(\mathrm{~m}, 1 \mathrm{H}), 1.55(\mathrm{~d}, 1 \mathrm{H}, J=14.0 \mathrm{~Hz}), 1.35$ (s, $3 \mathrm{H}), 1.17(\mathrm{~s}, 3 \mathrm{H}), 1.07(\mathrm{~s}, 3 \mathrm{H}) ;{ }^{13} \mathrm{C}$ NMR $\left(125 \mathrm{MHz}, \mathrm{CDCl}_{3}\right) \delta 141.8,135.9,135.2,128.3,126.8$, 126.1, 81.6, 65.4, 45.34, 45.32, 32.7, 32.4, 31.6, 29.5, 28.9; IR (neat, $\mathrm{cm}^{-1}$ ) 3055, 3021, 2953, 2923, 2861, 1598, 1444, 1358; MS (EI) Calculated for $\left[\mathrm{C}_{17} \mathrm{H}_{22} \mathrm{O}\right]^{+}: 242.17$; Found: 242.

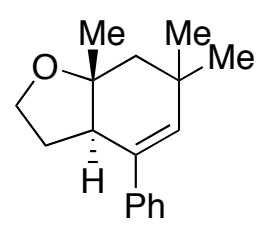

19

5,6-trans diastereomer 19: ${ }^{1} \mathrm{H}$ NMR $\left(500 \mathrm{MHz}, \mathrm{CDCl}_{3}\right) \delta$ 7.33-7.22 $(\mathrm{m}, 5 \mathrm{H}), 5.49(\mathrm{~d}, 1 \mathrm{H}, J=2.8$ $\mathrm{Hz}), 4.03-3.97(\mathrm{~m}, 2 \mathrm{H}), 2.80(\mathrm{ddd}, 1 \mathrm{H}, J=2.8,7.3,12.6 \mathrm{~Hz}), 2.23-2.17(\mathrm{~m}, 1 \mathrm{H}), 1.91(\mathrm{~d}, 1 \mathrm{H}, J=$ $12.4 \mathrm{~Hz}), 1.77(\mathrm{~d}, 1 \mathrm{H}, J=12.4 \mathrm{~Hz}), 1.73-1.64(\mathrm{~m}, 1 \mathrm{H}), 1.19(\mathrm{~s}, 3 \mathrm{H}), 1.16(\mathrm{~s}, 3 \mathrm{H}), 1.05(\mathrm{~s}, 3 \mathrm{H}) ;{ }^{13} \mathrm{C}$ NMR $\left(125 \mathrm{MHz}, \mathrm{CDCl}_{3}\right) \delta 141.3,136.8,128.1,126.7,126.6,82.8,66.0,49.5,46.9,35.7,33.1$, 31.1, 27.0, 18.6; IR (neat, $\mathrm{cm}^{-1}$ ) 3080, 3055, 2957, 2930, 2870, 1599, 1490, 1459, 1371; MS (EI) Calculated for $\left[\mathrm{C}_{17} \mathrm{H}_{22} \mathrm{O}\right]^{+}:$242.17; Found: 242.

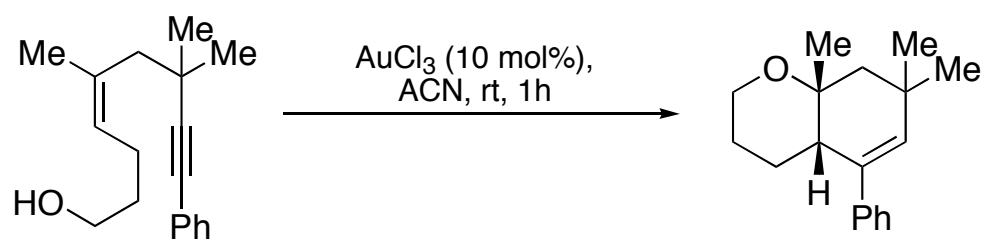

20

21

Alkene 21 was formed in $90 \%$ yield according to the general procedure $\mathrm{A}\left(10 \mathrm{~mol} \% \mathrm{AuCl}_{3}\right) .{ }^{1} \mathrm{H}$ NMR $\left(500 \mathrm{MHz}, \mathrm{CDCl}_{3}\right) \delta$ 7.36-7.24 (m, 5H), $5.54(\mathrm{~s}, 1 \mathrm{H}), 3.76-3.70(\mathrm{~m}, 2 \mathrm{H}), 2.43(\mathrm{dd}, 1 \mathrm{H}, J=$ 4.3, 10.6 Hz), $2.26(\mathrm{~d}, 1 \mathrm{H}, J=13.5 \mathrm{~Hz}), 1.77-1.73(\mathrm{~m}, 1 \mathrm{H}), 1.53-1.47(\mathrm{~m}, 2 \mathrm{H}), 1.38(\mathrm{~s}, 3 \mathrm{H}), 1.37-$ $1.32(\mathrm{~m}, 1 \mathrm{H}), 1.31(\mathrm{~d}, 1 \mathrm{H}, J=13.5 \mathrm{~Hz}), 1.19(\mathrm{~s}, 3 \mathrm{H}), 1.12(\mathrm{~s}, 3 \mathrm{H}) ;{ }^{13} \mathrm{C} \mathrm{NMR}\left(125 \mathrm{MHz}, \mathrm{CDCl}_{3}\right) \delta$ 141.7, 138.2, 128.2, 126.7, 126.3, 73.7, 60.9, 43.0, 40.3, 33.6, 32.9, 30.3, 28.5, 26.7; IR (neat, 
$\left.\mathrm{cm}^{-1}\right) 3055,2935,2860,1599,1493,1468,1444,1375$; MS (APCl) Calculated for $\left[\mathrm{C}_{18} \mathrm{H}_{24} \mathrm{O}\right]^{+}$: 256.18; Found: 256.

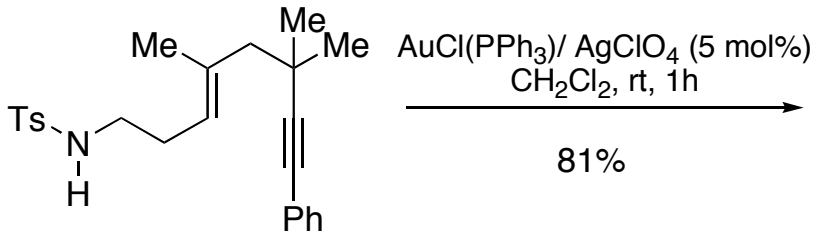

22

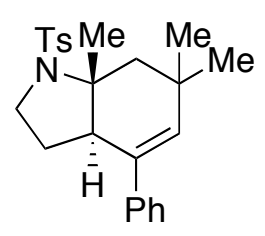

23

Alkene 23 was obtained in $81 \%$ yield according to the general procedure B. ${ }^{1} \mathrm{H}$ NMR $(500 \mathrm{MHz}$, $\left.\mathrm{CDCl}_{3}\right) \delta 7.76(\mathrm{~d}, 2 \mathrm{H}, J=8.1 \mathrm{~Hz}), 7.30-7.26(\mathrm{~m}, 3 \mathrm{H}), 7.22(\mathrm{t}, 1 \mathrm{H}, J=7.2 \mathrm{~Hz}), 7.15(\mathrm{~d}, 2 \mathrm{H}, J=7.2$ $\mathrm{Hz}), 5.46(\mathrm{~d}, 1 \mathrm{H}, J=2.6 \mathrm{~Hz}), 3.48(\mathrm{t}, 1 \mathrm{H}, J=9.5 \mathrm{~Hz}), 3.39(\mathrm{q}, 1 \mathrm{H}, J=8.8 \mathrm{~Hz}), 2.92(1 \mathrm{H}, \mathrm{ddd}, J=$ 2.6, 6.4, $13.5 \mathrm{~Hz}$ ), $2.58(\mathrm{~d}, 1 \mathrm{H}, J=13.2 \mathrm{~Hz}), 2.42(\mathrm{~s}, 3 \mathrm{H}), 2.10-2.04(\mathrm{~m}, 1 \mathrm{H}), 1.91(\mathrm{~d}, 1 \mathrm{H}, J=13.5$ $\mathrm{Hz}), 1.53-1.43(\mathrm{~m}, 1 \mathrm{H}), 1.17(\mathrm{~s}, 3 \mathrm{H}), 1.12(\mathrm{~s}, 6 \mathrm{H}) ;{ }^{13} \mathrm{C} \mathrm{NMR}\left(125 \mathrm{MHz}, \mathrm{CDCl}_{3}\right) \delta$ 142.89, 140.9, 138.2, 137.3, 135.3, 129.4, 128.1, 127.4,m 127.0, 126.8, 68.0, 49.4, 48.8, 47.2, 35.0, 33.1, 31.0, 24.5, 21.5, 18.7; IR (neat, $\mathrm{cm}^{-1}$ ) 2924, 1599, 1457, 1334; MS (APCI) Calculated for $\left[\mathrm{C}_{24} \mathrm{H}_{30} \mathrm{NO}_{2} \mathrm{~S}\right]^{+}$: 396.57; Found: 396.1.

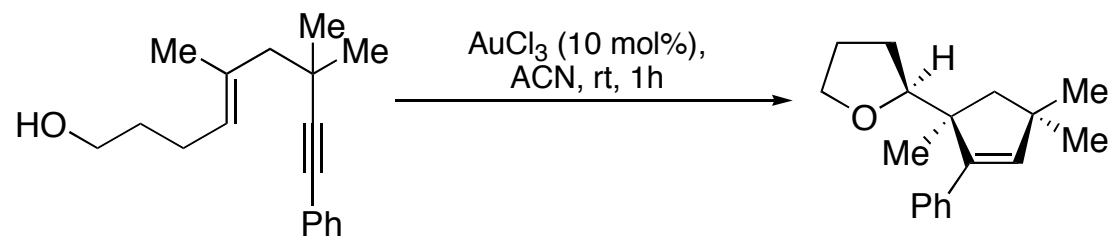

24

25

Alkene 25 was formed in $98 \%$ yield according to the general procedure $\mathrm{A}\left(10 \mathrm{~mol} \% \mathrm{AuCl}_{3}\right.$ ). ${ }^{1} \mathrm{H}$ NMR (500 MHz, toluene-d8) $8.40(\mathrm{~d}, 2 \mathrm{H}, J=8.3 \mathrm{~Hz}), 7.18(\mathrm{~d}, 2 \mathrm{H}, J=8.3 \mathrm{~Hz}), 7.11(\mathrm{t}, 1 \mathrm{H}, J=$ $8.3 \mathrm{~Hz}$ ), $5.36(\mathrm{~s}, 1 \mathrm{H}), 3.68-3.63(\mathrm{~m}, 1 \mathrm{H}), 3.59(\mathrm{dd}, 1 \mathrm{H}, J=5.9,9.4 \mathrm{~Hz}), 3.54-3.51(\mathrm{~m}, 1 \mathrm{H}), 2.06(\mathrm{~d}$, $1 \mathrm{H}, J=12.7 \mathrm{~Hz}), 1.54-1.40(\mathrm{~m}, 4 \mathrm{H}), 12.7-1.22(\mathrm{~m}, 1 \mathrm{H}), 1.217(\mathrm{~s}, 3 \mathrm{H}), 1.207(\mathrm{~s}, 3 \mathrm{H}), 1.09(\mathrm{~s}, 3 \mathrm{H})$; ${ }^{13} \mathrm{C}$ NMR (125 MHz, toluene-d8) $\delta 147.9,139.0,138.8,129.4,127.9,126.9,82.9,68.1,55.2$, 47.4, 42.5, 31.1, 30.2, 27.7, 26.3; IR (neat, $\mathrm{cm}^{-1}$ ) 3020, 2956, 2925, 2860, 1598, 1462, 1441, 1371; MS (APCI) Calculated for $\left[\mathrm{C}_{18} \mathrm{H}_{24} \mathrm{O}\right]^{+}:$256.18; Found: 256. 
<smiles>C=C(C)CC(C)(C#Cc1ccccc1)CO</smiles>

1

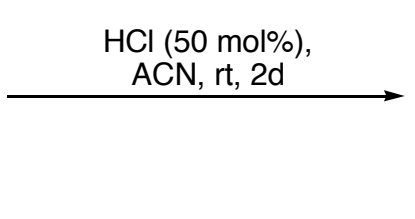

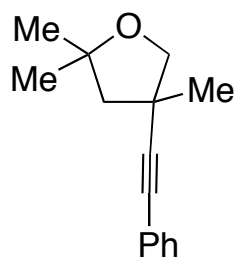

3

Tetrahydrofuran 3. To a NMR tube were added alcohol 1 (7.3 $\mathrm{mg}, 0.034 \mathrm{mmol}), \mathrm{CD}_{3} \mathrm{CN}(0.5$ $\mathrm{mL})$ and $\mathrm{HCl}$ in $\mathrm{Et}_{2} \mathrm{O}(1 \mathrm{M}, 17 \mu \mathrm{L}, 0.017 \mathrm{mmol})$. The resulting mixture was allow to stand for 2 days until all the starting material was consumed as indicated by ${ }^{1} \mathrm{H}$ NMR. The reaction mixture was neutralized with $\mathrm{Et}_{3} \mathrm{~N}(50 \mu \mathrm{L})$ and concentrated under vacuum. Silica gel flash column chromatography gave compound 3 in $80 \%$ yield. ${ }^{1} \mathrm{H}$ NMR $\left(500 \mathrm{MHz}, \mathrm{CDCl}_{3}\right) \delta 7.40-7.38(\mathrm{~m}, 2 \mathrm{H})$, 7.29-7.27 (m, 3H), $4.03(\mathrm{~d}, 1 \mathrm{H}, J=8.5 \mathrm{~Hz}), 3.70(\mathrm{~d}, 1 \mathrm{H}, J=8.5 \mathrm{~Hz}), 2.24(\mathrm{~d}, 1 \mathrm{H}, J=12.6 \mathrm{~Hz})$, $1.81(\mathrm{~d}, 1 \mathrm{H}, J=12.6 \mathrm{~Hz}), 1.45(\mathrm{~s}, 3 \mathrm{H}), 1.39(\mathrm{~s}, 3 \mathrm{H}), 1.33(\mathrm{~s}, 3 \mathrm{H}) ;{ }^{13} \mathrm{C}$ NMR $\left(125 \mathrm{MHz}, \mathrm{CDCl}_{3}\right) \delta$ 131.5, 128.2, 127.7, 123.6, 95.4, 81.6, 80.7, 78.2, 53.5, 39.4, 29.4, 28.4, 26.5; IR (neat, $\mathrm{cm}^{-1}$ ) 2970, 2930, 2869, 1599, 1492, 1445, 1366; MS (EI) Calculated for $\left[\mathrm{C}_{15} \mathrm{H}_{18} \mathrm{O}\right]^{+}: 214.14$; Found: 214.

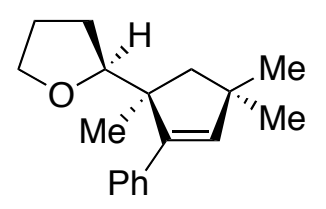

25
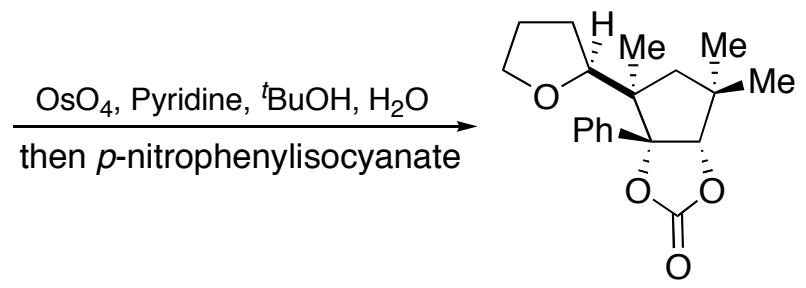

X-ray analysis

26

Carbonate 26. Its structure was verified by X-Ray analysis, see page S-72. Alkene 25 (15.6 mg, $0.061 \mathrm{mmol})$ was mixed with pyridine $(0.2 \mathrm{~mL}), \mathrm{H}_{2} \mathrm{O}(1.5 \mathrm{~mL}),{ }^{\mathrm{t}} \mathrm{BuOH}(4 \mathrm{~mL})$ and $\mathrm{OsO}_{4}(2.5 \mathrm{w} \%$ in ${ }^{t} \mathrm{BuOH}, 2 \mathrm{~mL}$ ). The resulting solution was heated to reflux for $24 \mathrm{~h}$ before being cooled to room temperature and treated with saturated $\mathrm{Na}_{2} \mathrm{SO}_{3}$ aqueous solution $(5 \mathrm{~mL})$. ${ }^{t} \mathrm{BuOH}$ was removed under vacuum and the residue was saturated with $\mathrm{NaCl}$ and extracted with diethyl ether $(3 \times 10$ $\mathrm{mL})$. The combined organic extracts were dried $\left(\mathrm{MgSO}_{4}\right)$ and concentrated. Flash column chromatography yielded the diol as a clear liquid (13 $\mathrm{mg})$.

The diol was then reacted with $p$-nitrophenyl isocyanate $(7.5 \mathrm{mg})$ in refluxing $\mathrm{CH}_{2} \mathrm{Cl}_{2}(2 \mathrm{~mL})$ for 4 $\mathrm{h}$. The solvent was removed after the reaction and the residue was purified through flash column chromatography to yield carbonate 26 as a solid (5.2 mg, 27\%). $\left.{ }^{1} \mathrm{H} \mathrm{NMR} \mathrm{(500} \mathrm{MHz}, \mathrm{CDCl}_{3}\right) \delta$ 
7.61-7.58 (m, 2H), 7.38-7.32 (m, 3H), $5.00(\mathrm{~s}, 1 \mathrm{H}), 3.89(\mathrm{dd}, 1 \mathrm{H}, J=7.0,15.0 \mathrm{~Hz}), 3.51(\mathrm{dd}, 1 \mathrm{H}, J$ $=7.3,15.0 \mathrm{~Hz}), 2.88(\mathrm{dd}, 1 \mathrm{H}, J=6.4,9.3 \mathrm{~Hz}), 2.05(\mathrm{~d}, 1 \mathrm{H}, J=14.0 \mathrm{~Hz}), 1.79(\mathrm{~d}, 1 \mathrm{H}, J=14.0$ $\mathrm{Hz}), 1.73-1.69(\mathrm{~m}, 1 \mathrm{H}), 1.57-1.53(\mathrm{~m}, 1 \mathrm{H}), 1.45-1.34(\mathrm{~m}, 2 \mathrm{H}), 1.34(\mathrm{~s}, 3 \mathrm{H}), 1.20(\mathrm{~s}, 3 \mathrm{H}), 1.06(\mathrm{~s}$, $3 \mathrm{H}) ;{ }^{13} \mathrm{C}$ NMR $\left(125 \mathrm{MHz}, \mathrm{CDCl}_{3}\right) \delta 154.5,136.0,128.5,128.0,126.6,102.1,93.6,81.8,67.6$, 50.8, 48.9, 40.2, 29.6, 28.2, 27.0, 24.9, 18.6; IR (neat, $\mathrm{cm}^{-1}$ ) 2962, 2873, 1795, 1446, 1340. 


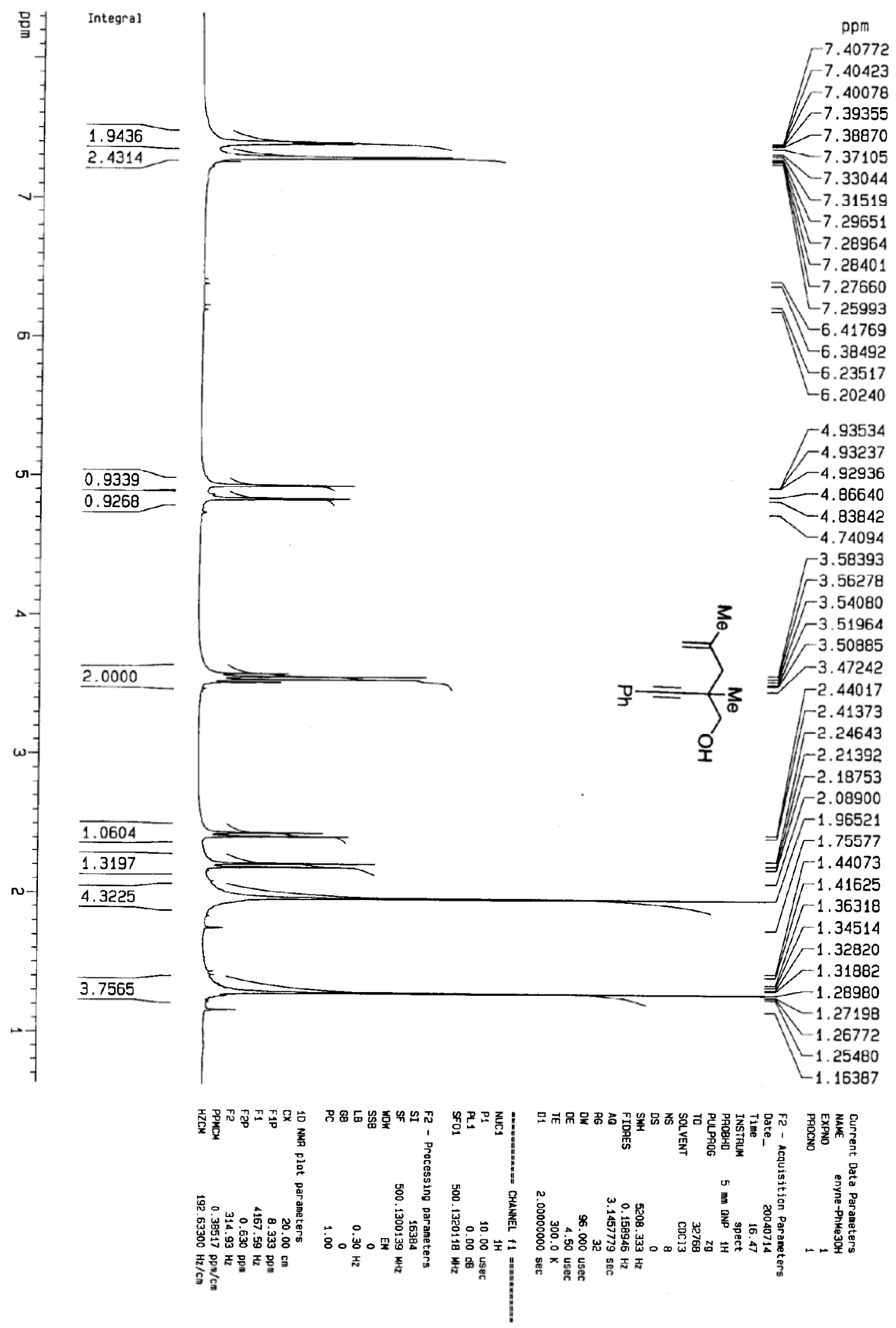




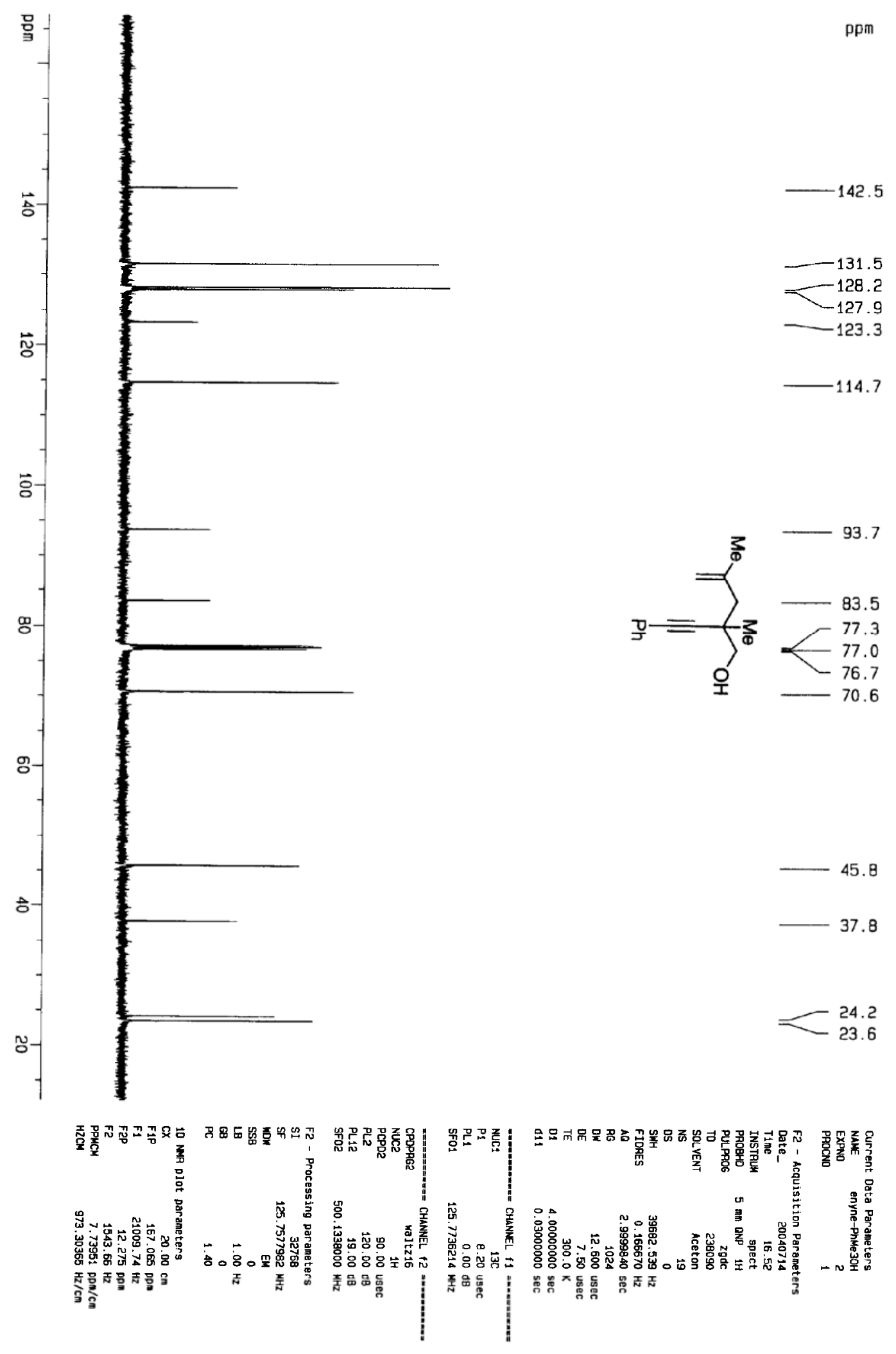




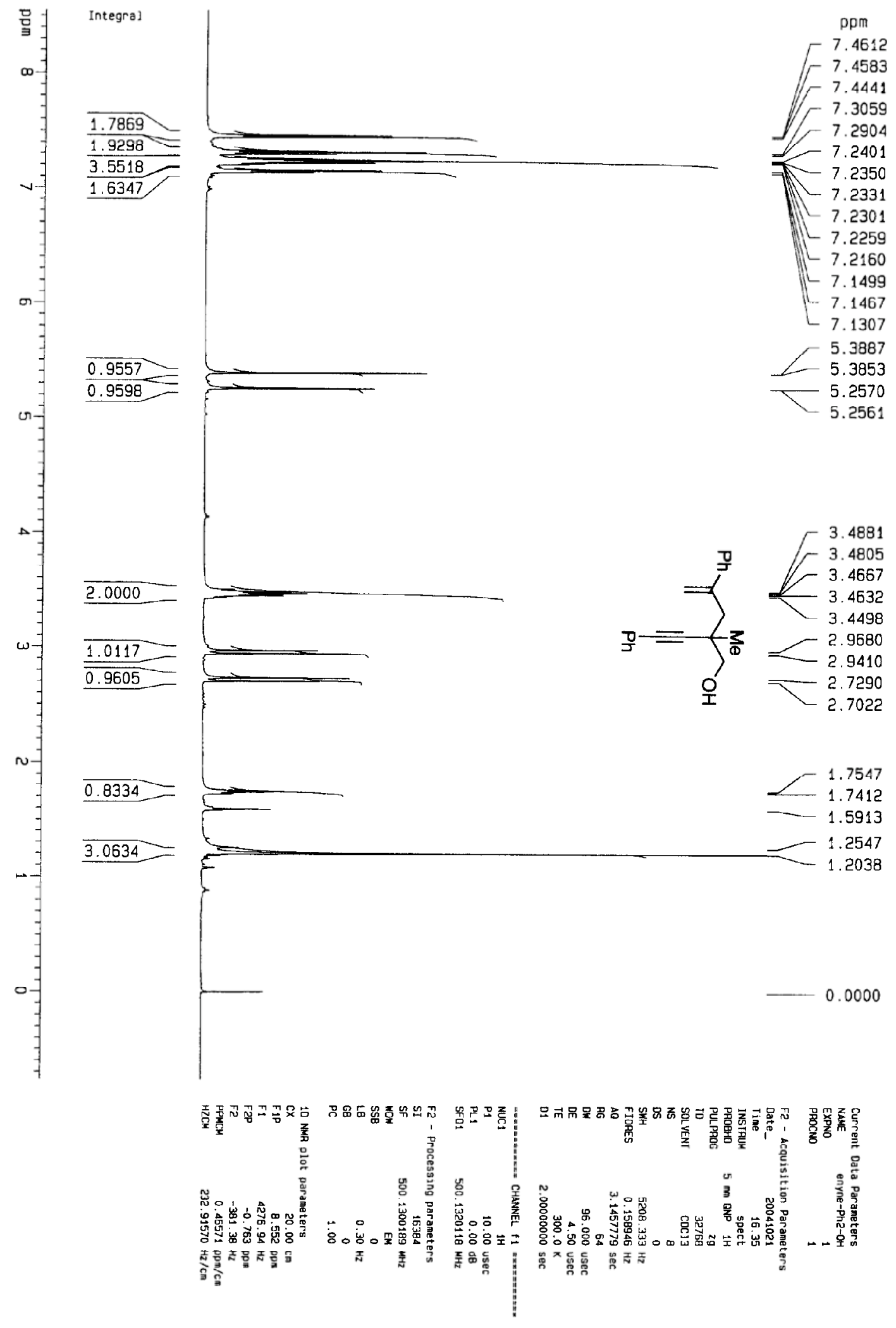




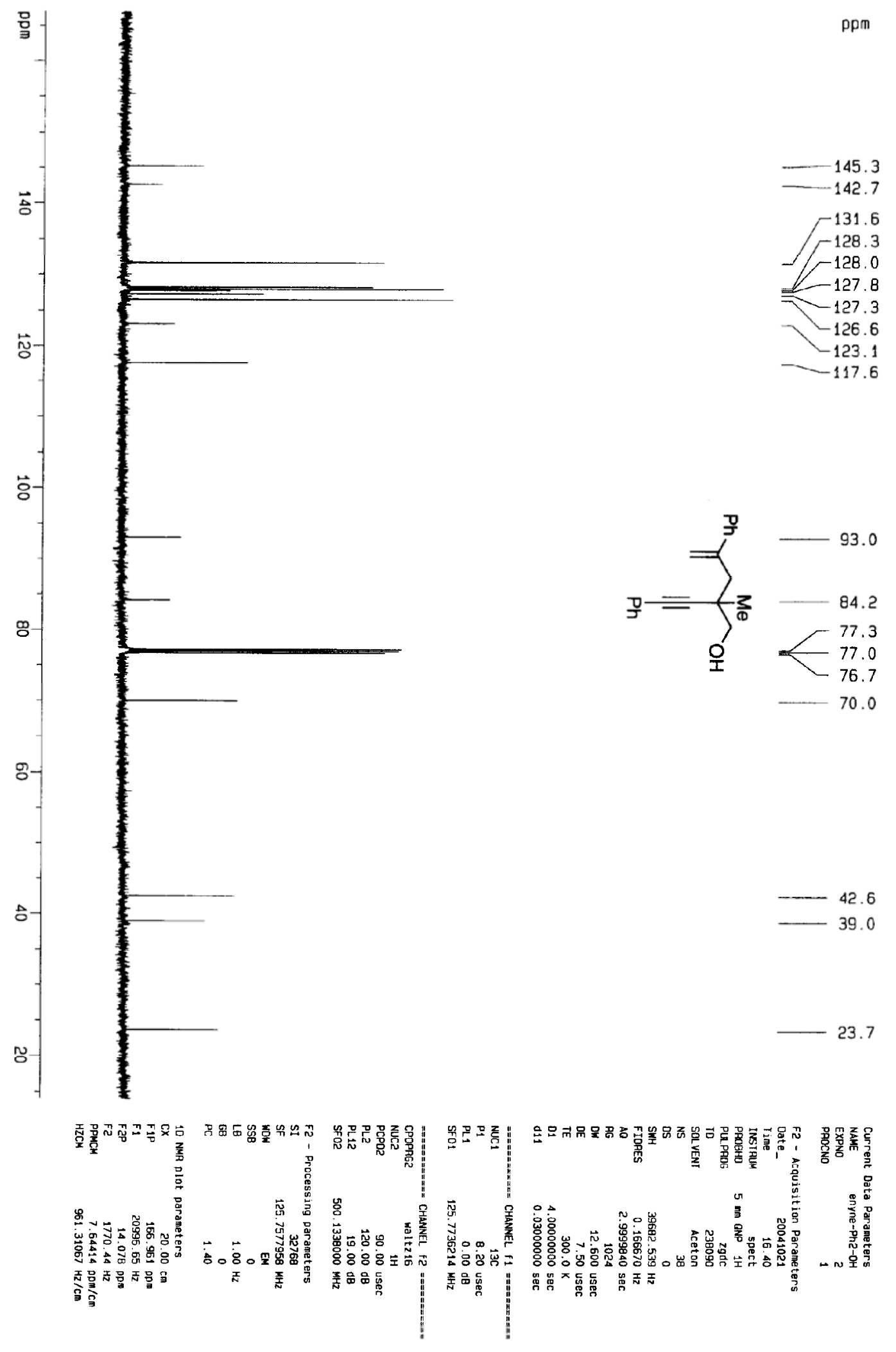




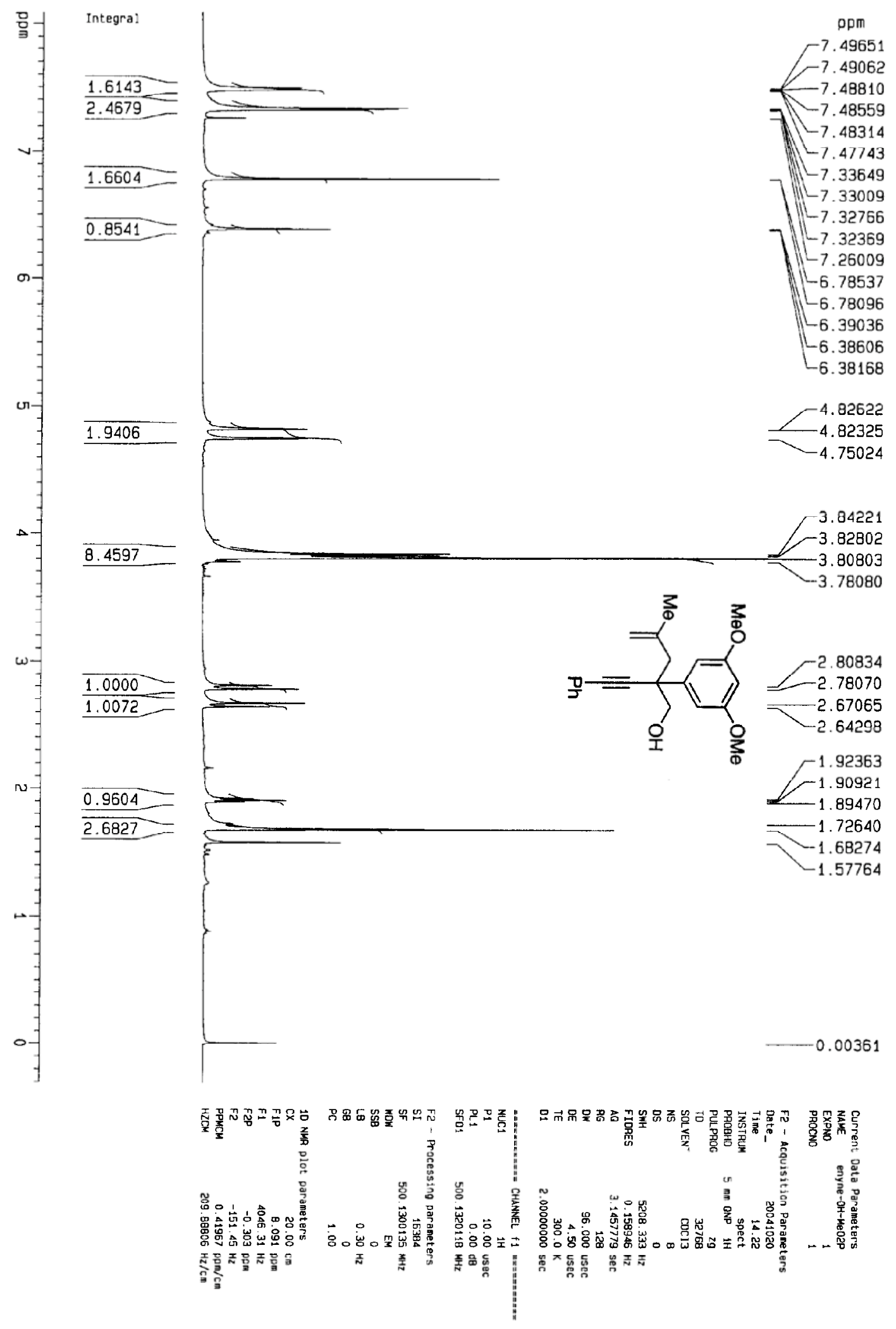




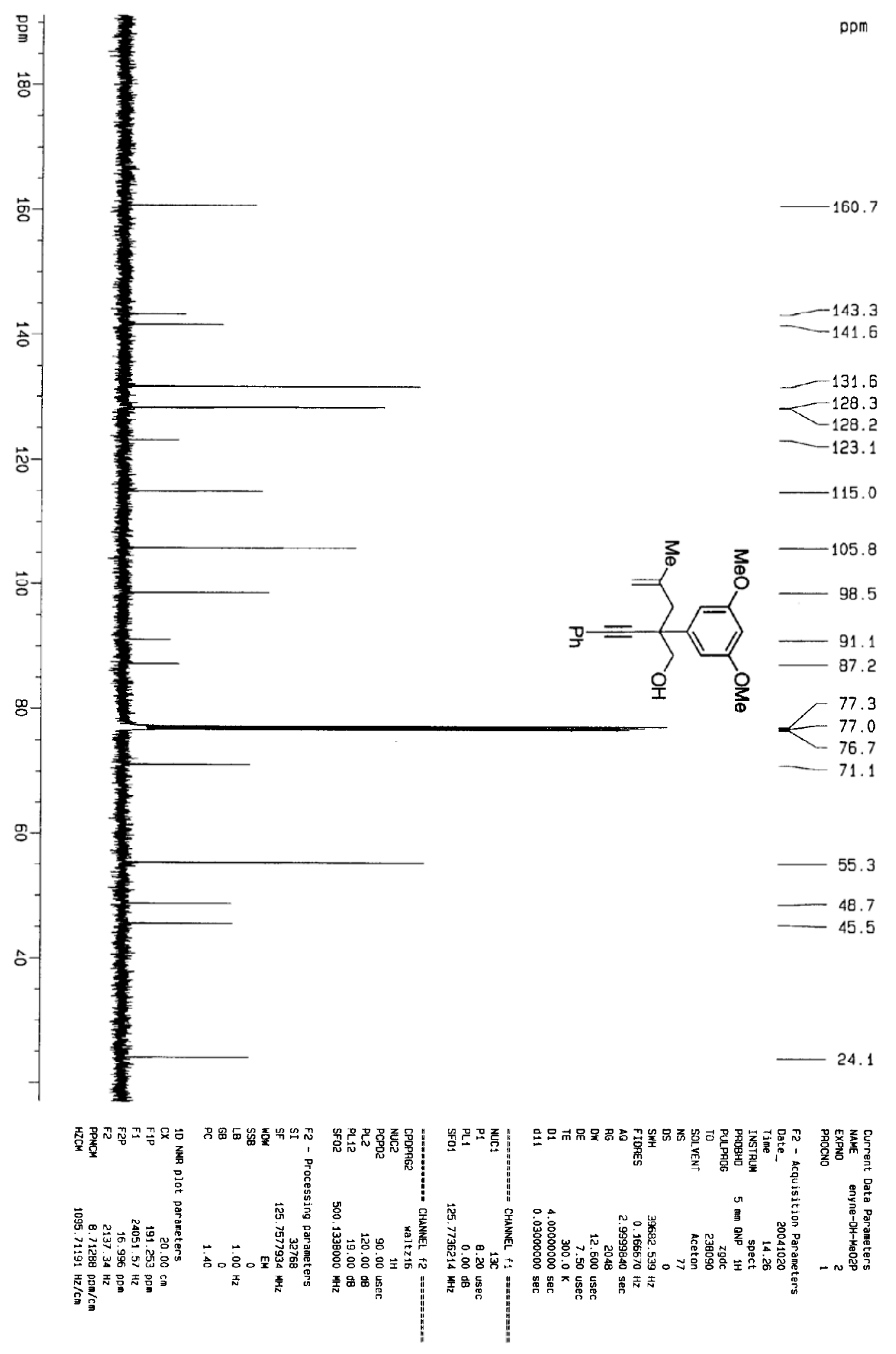




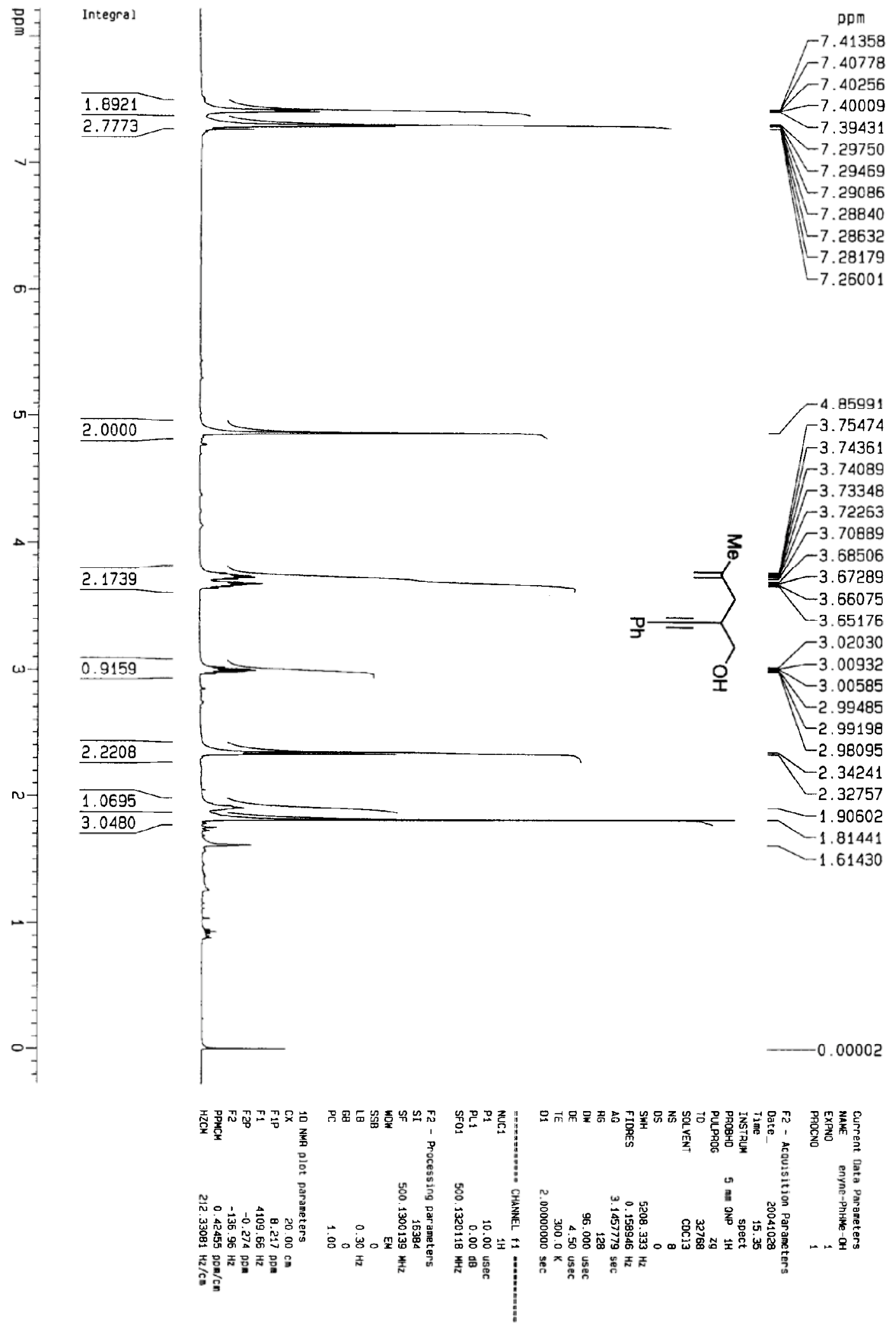




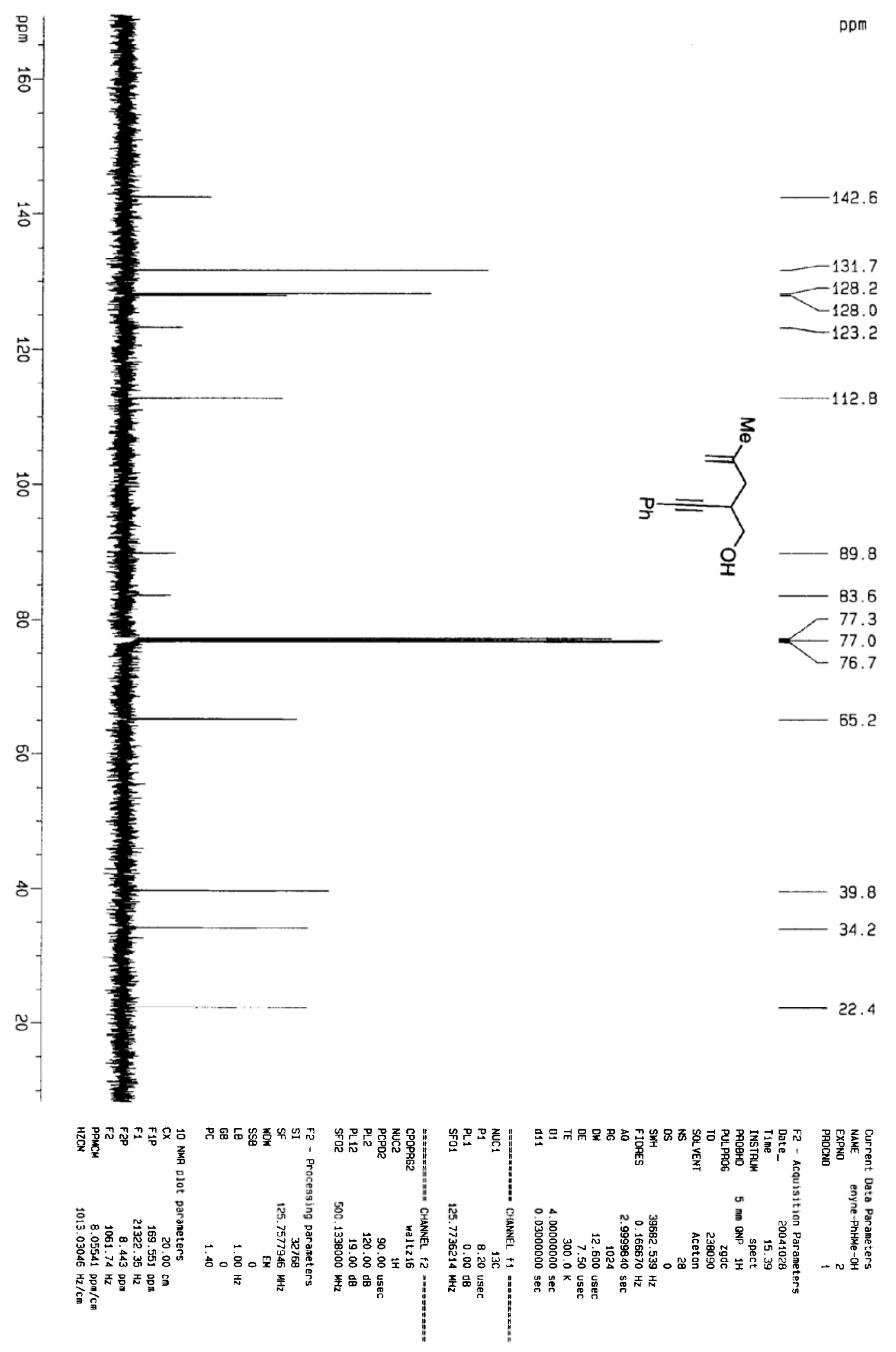




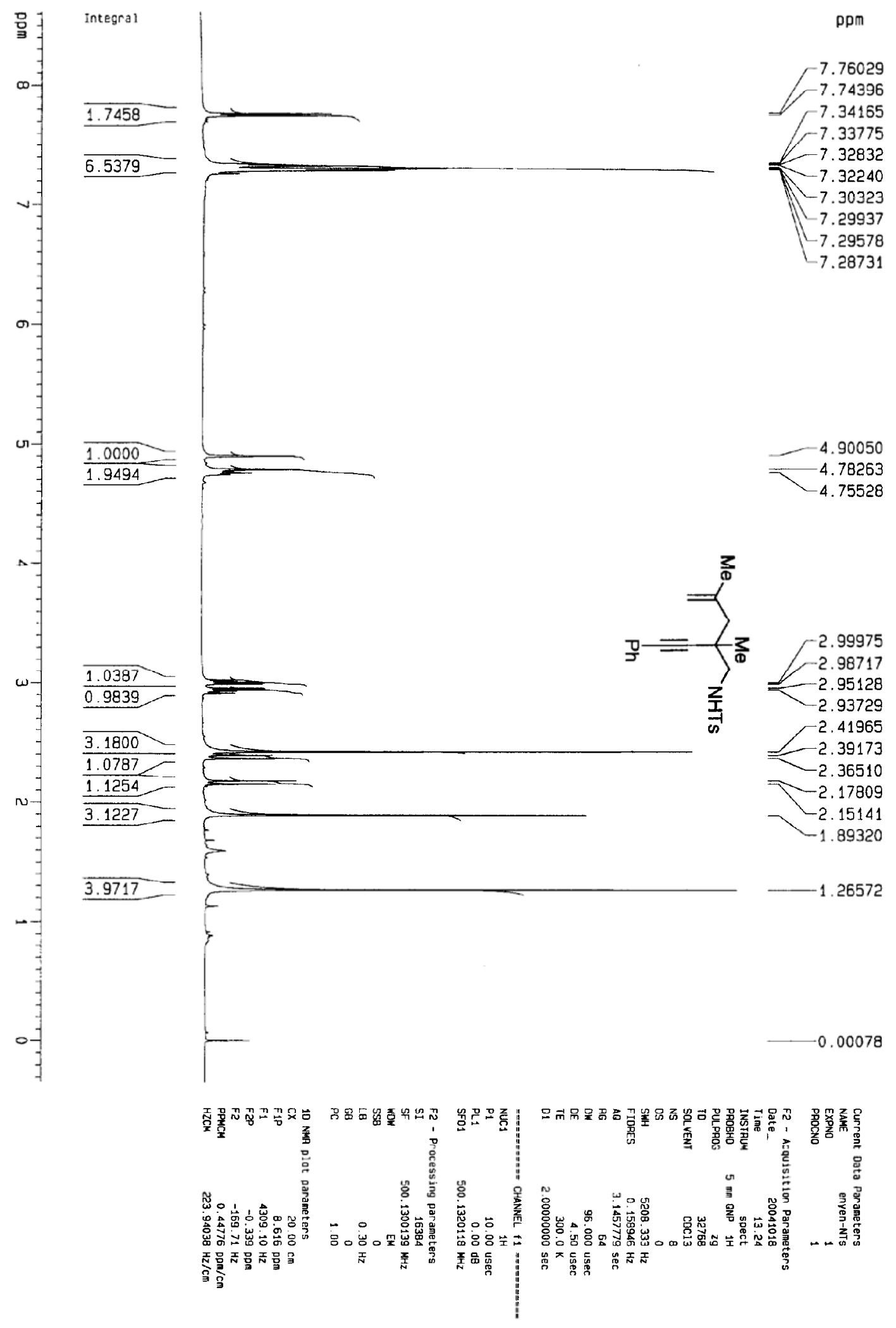




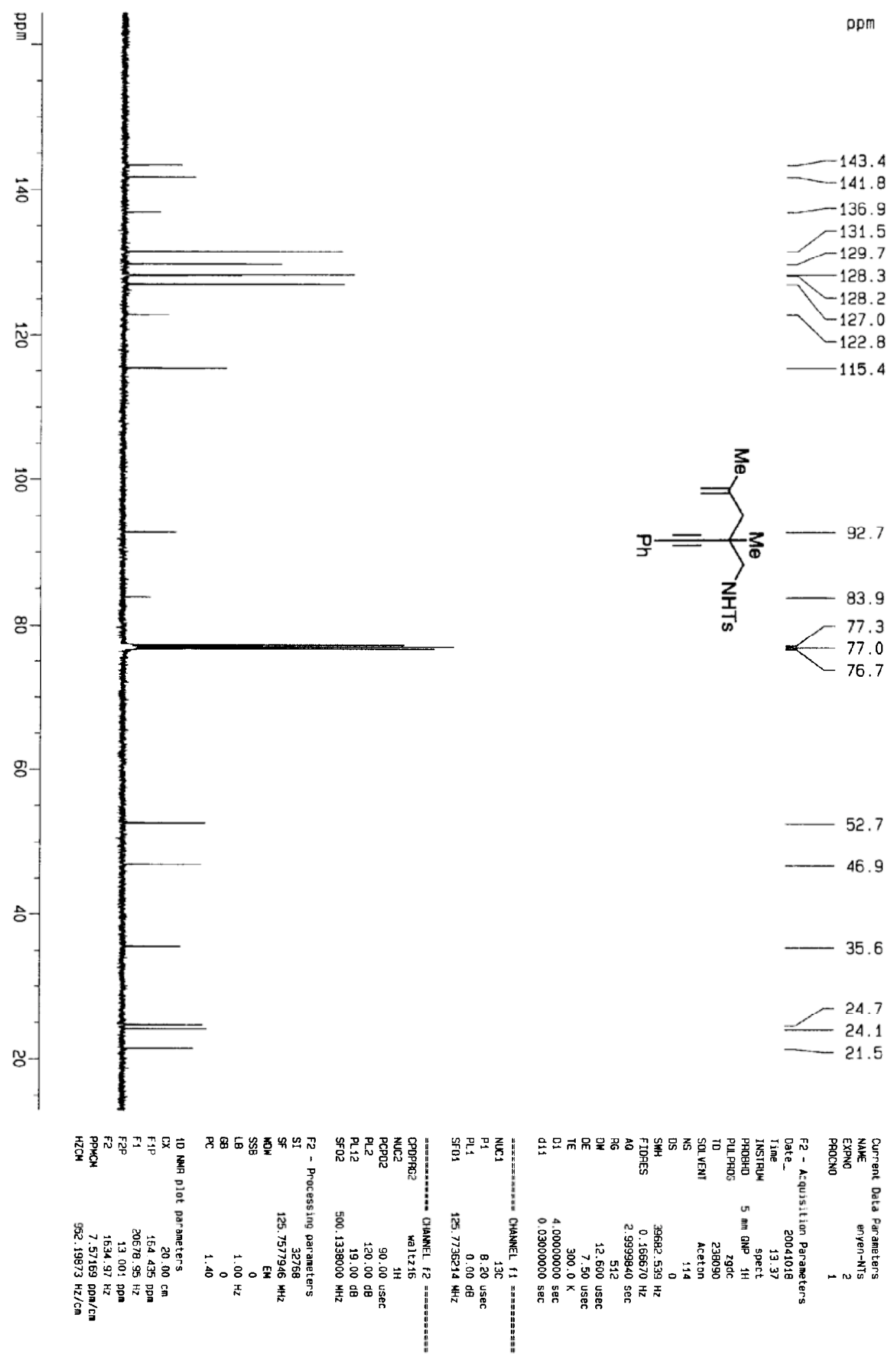




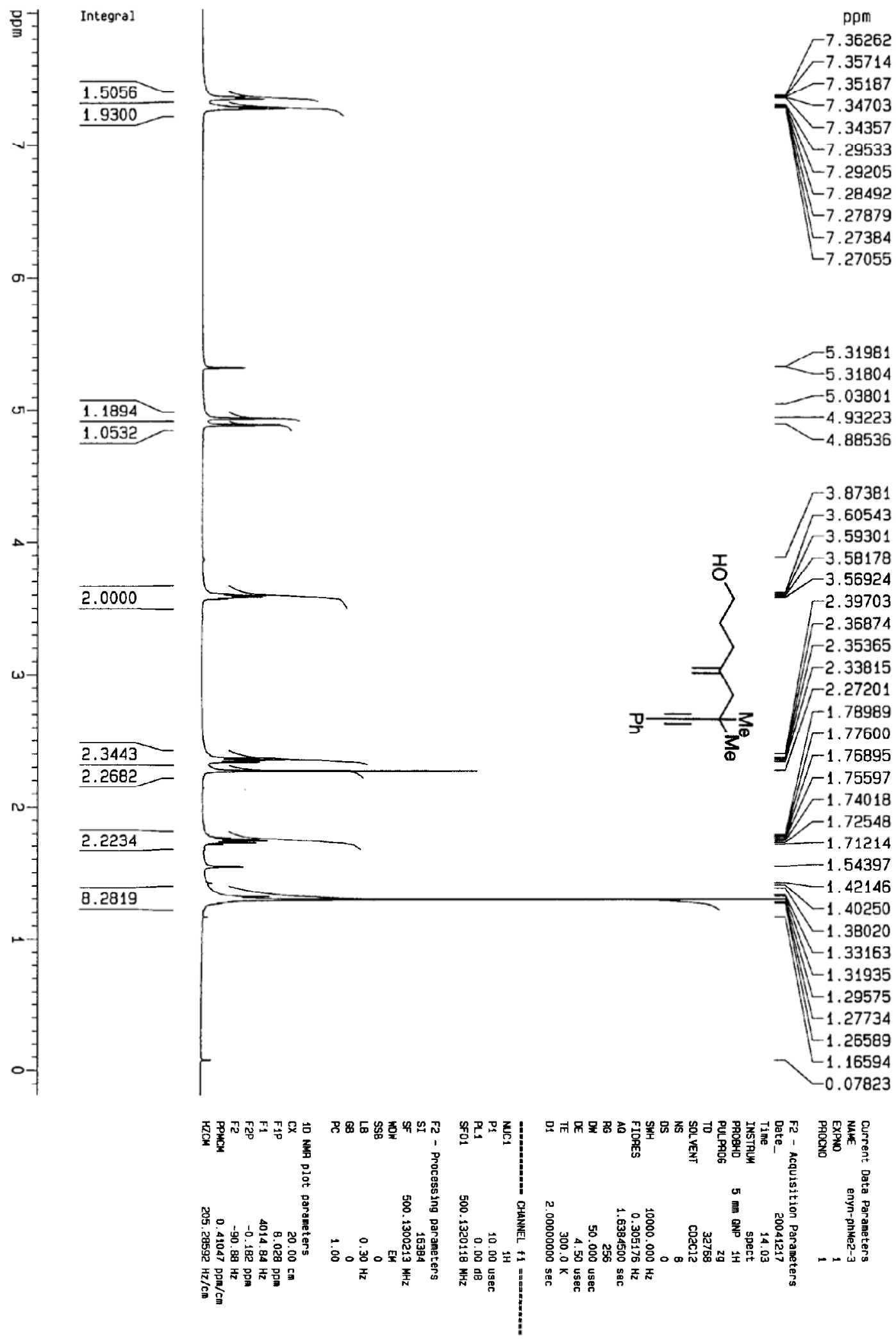




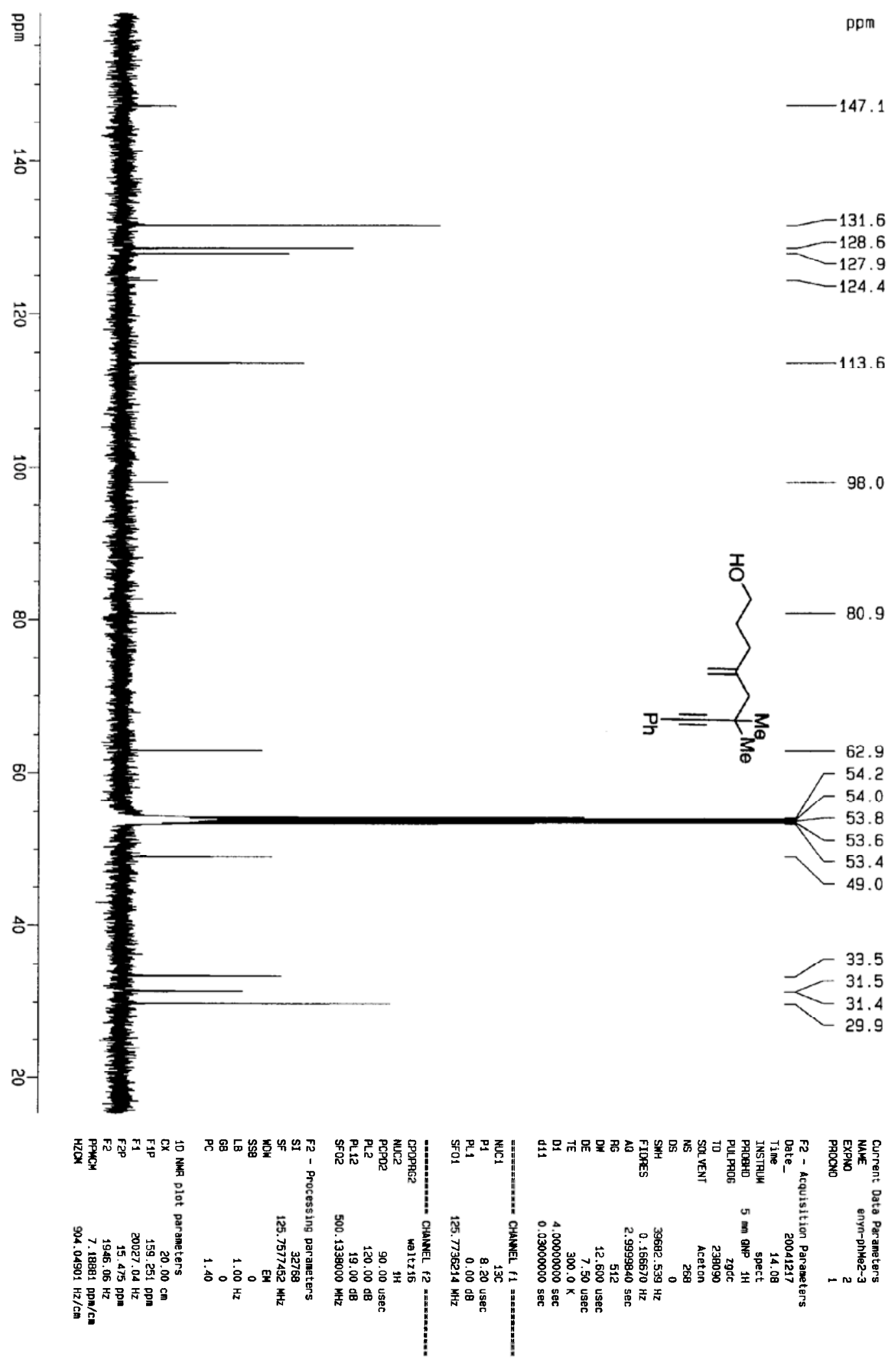




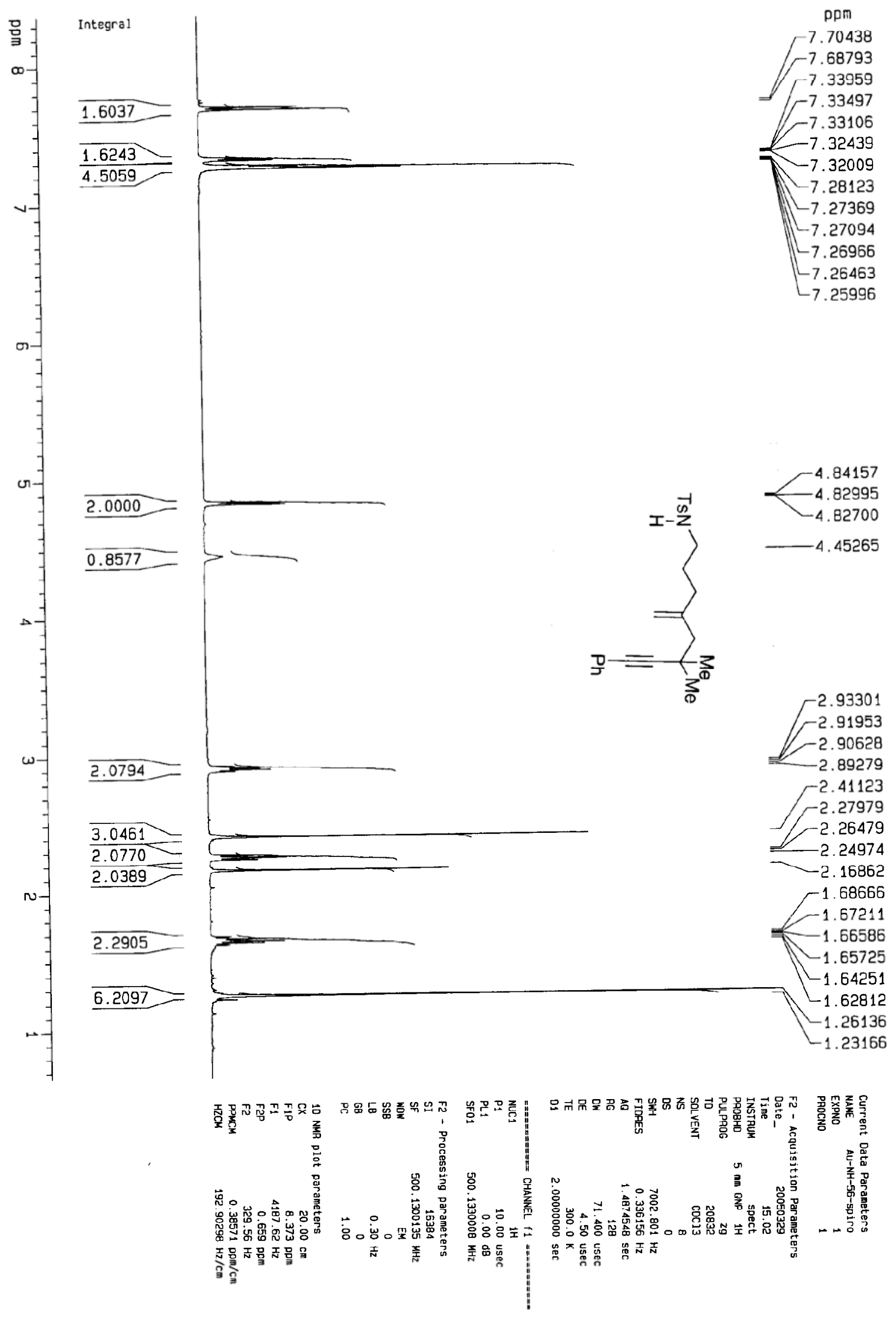




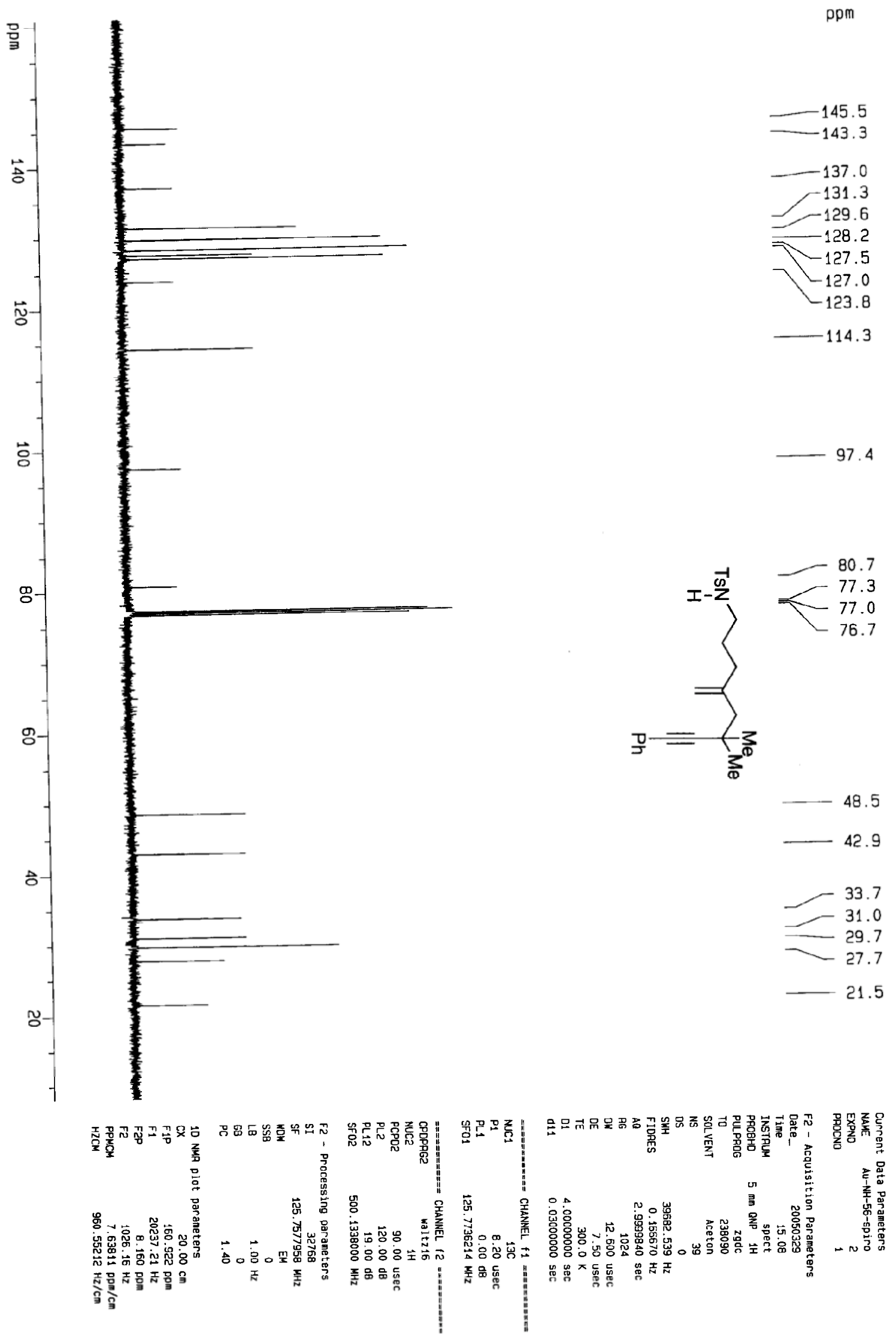




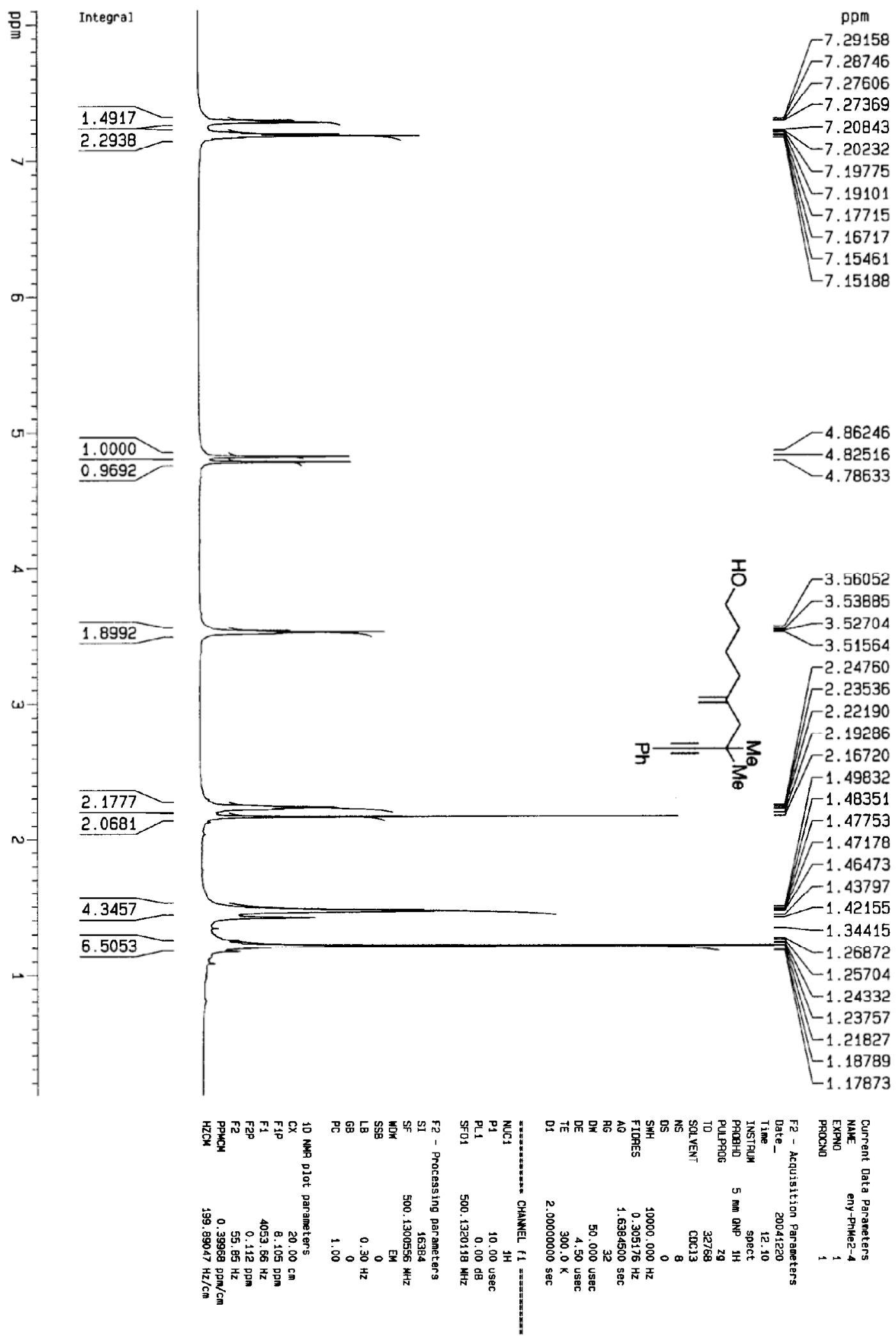




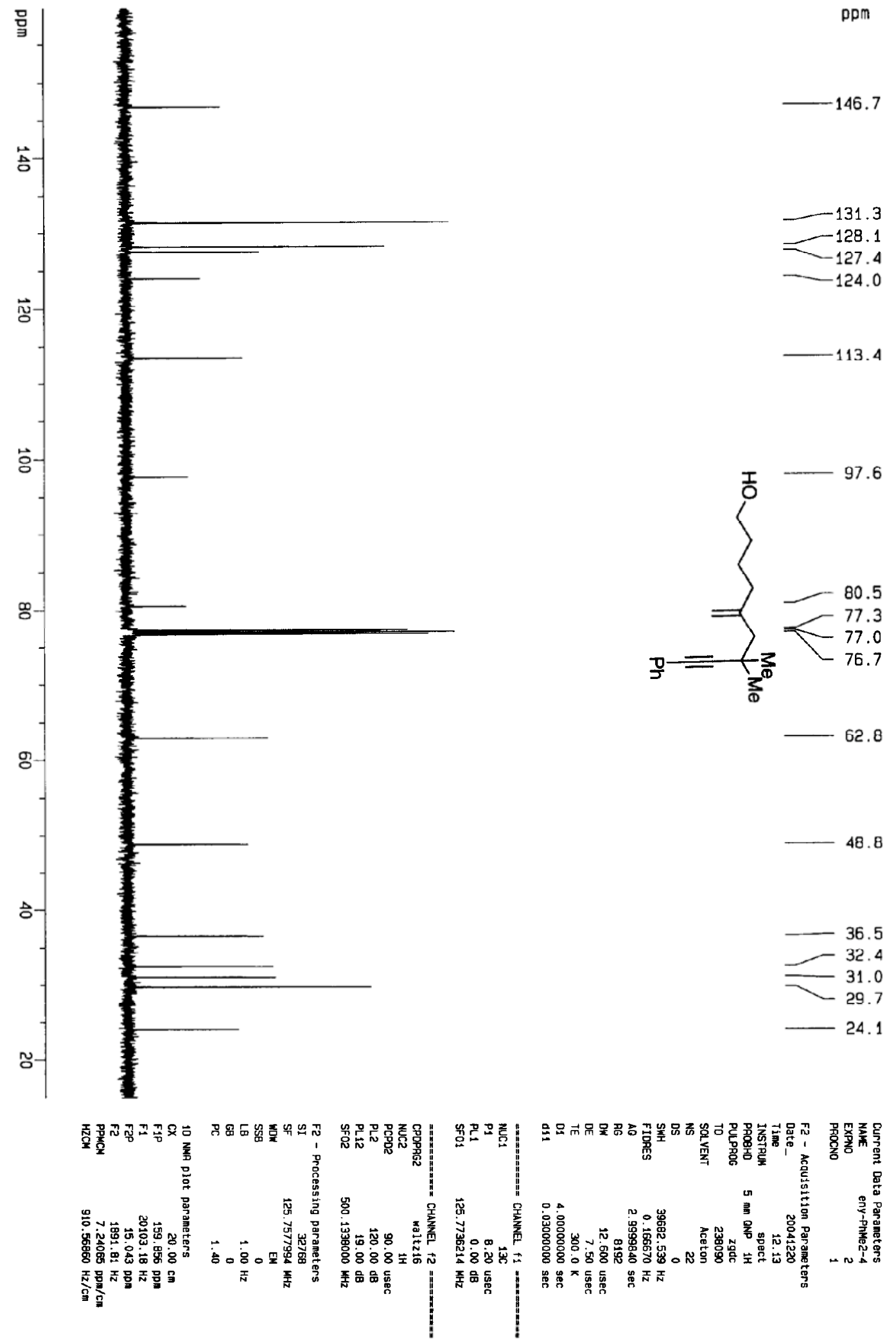




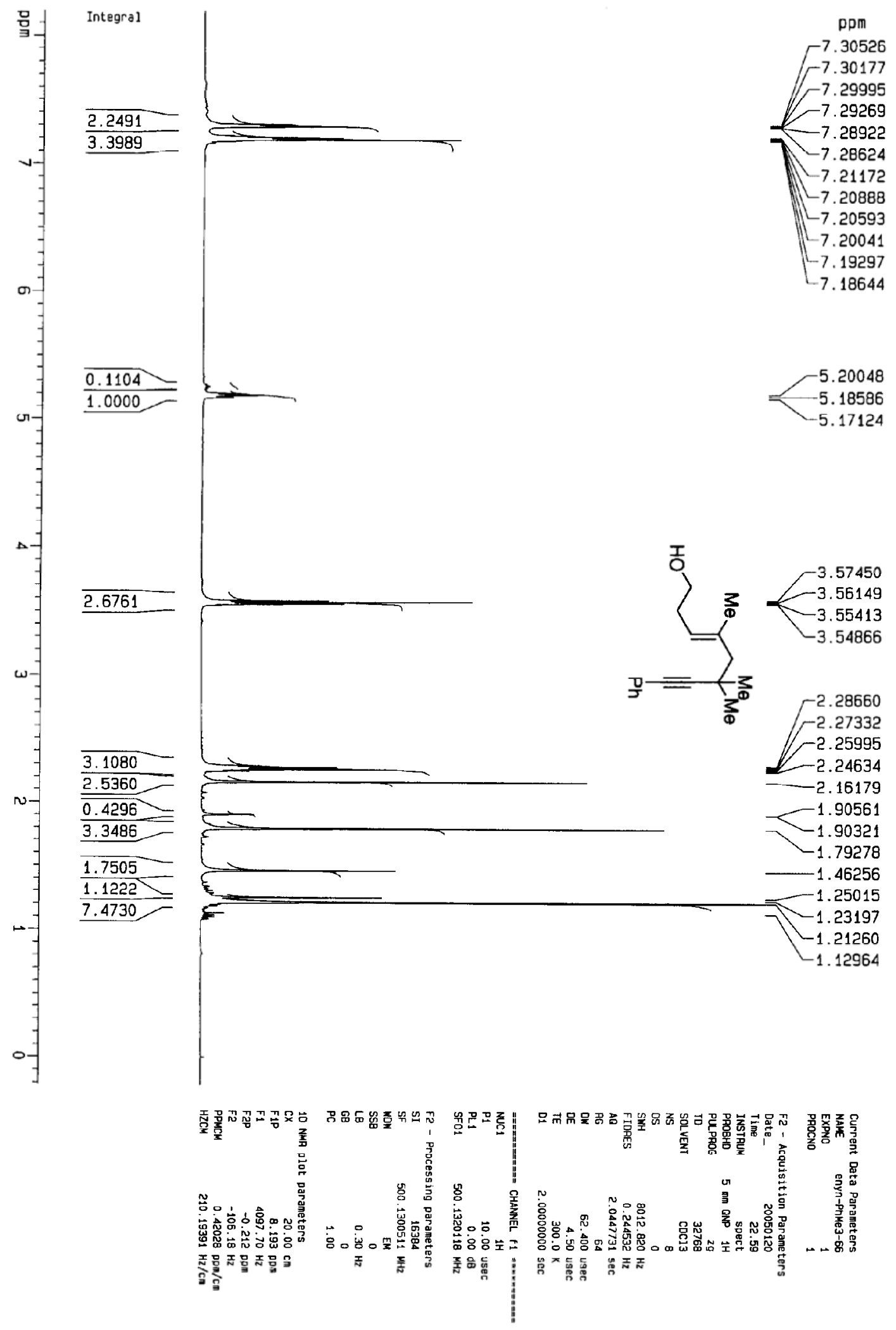




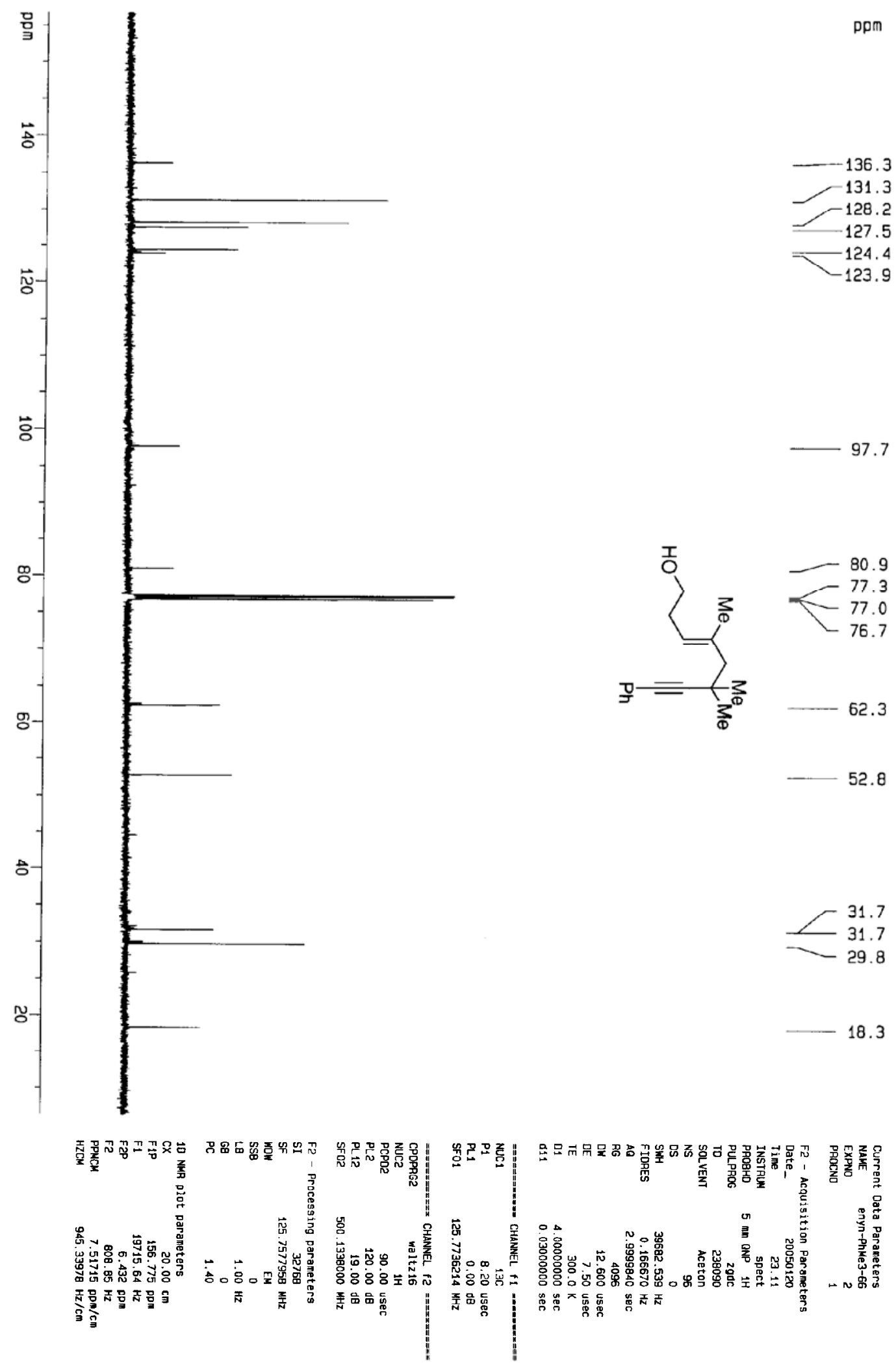




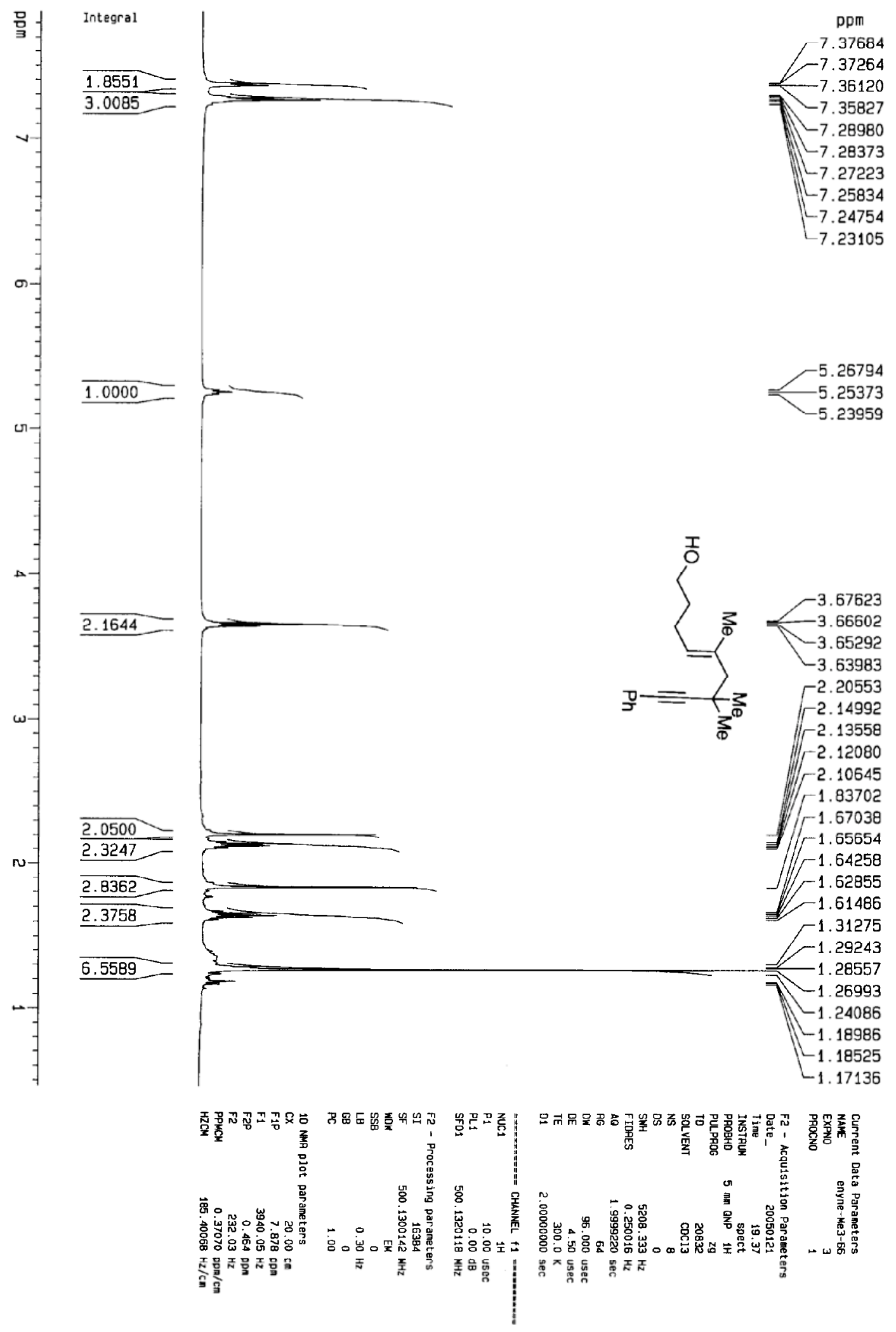




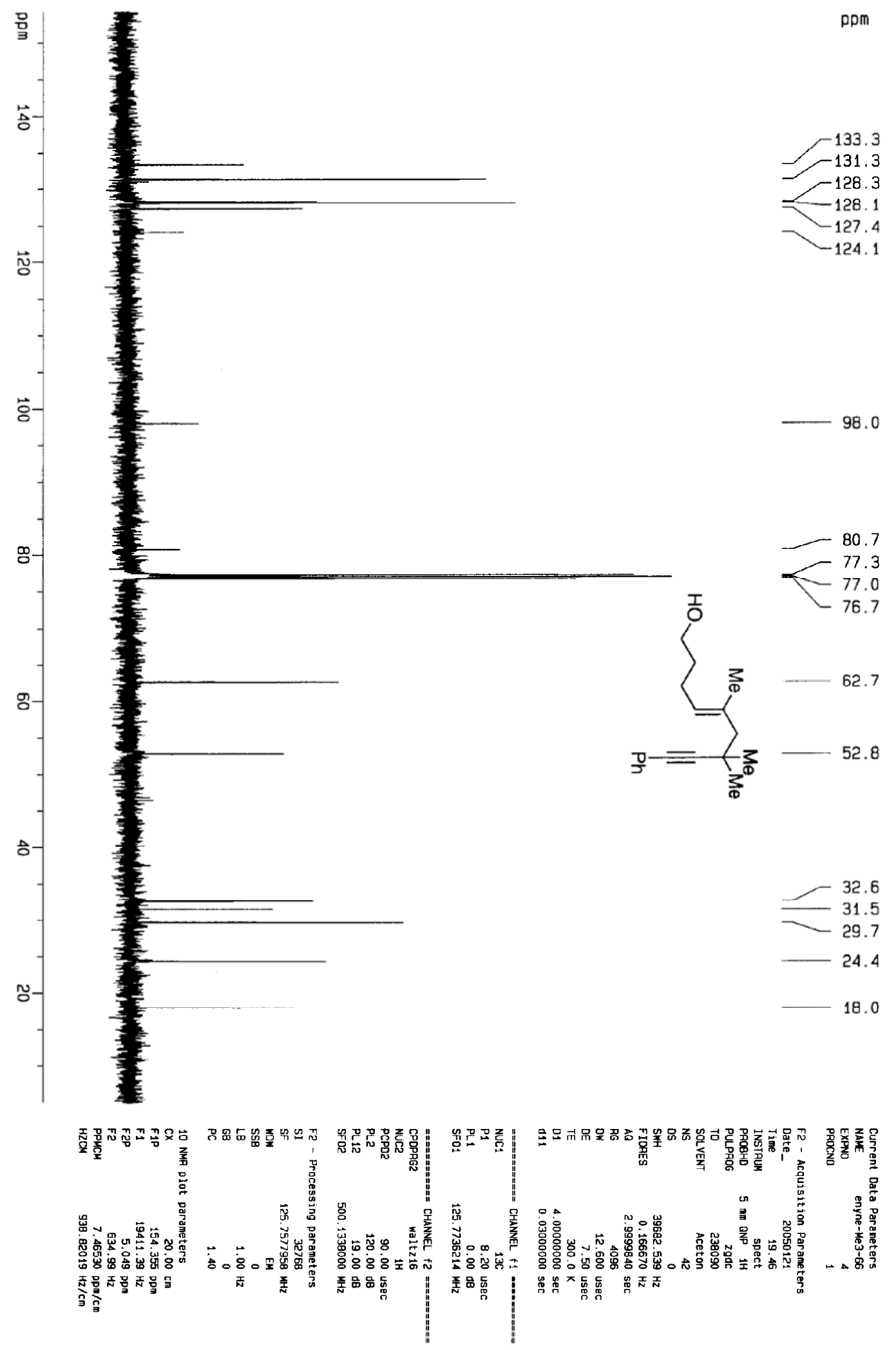




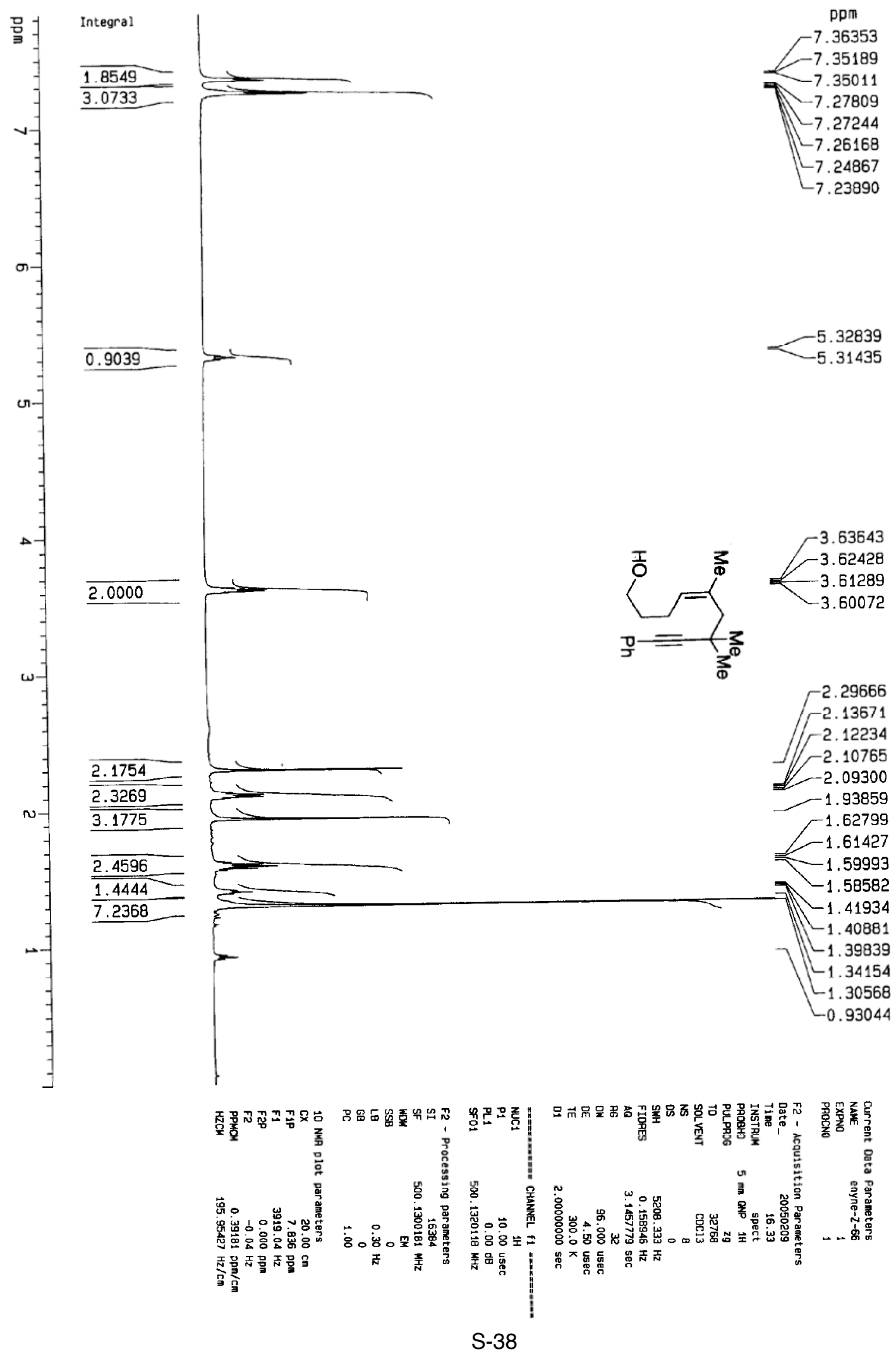



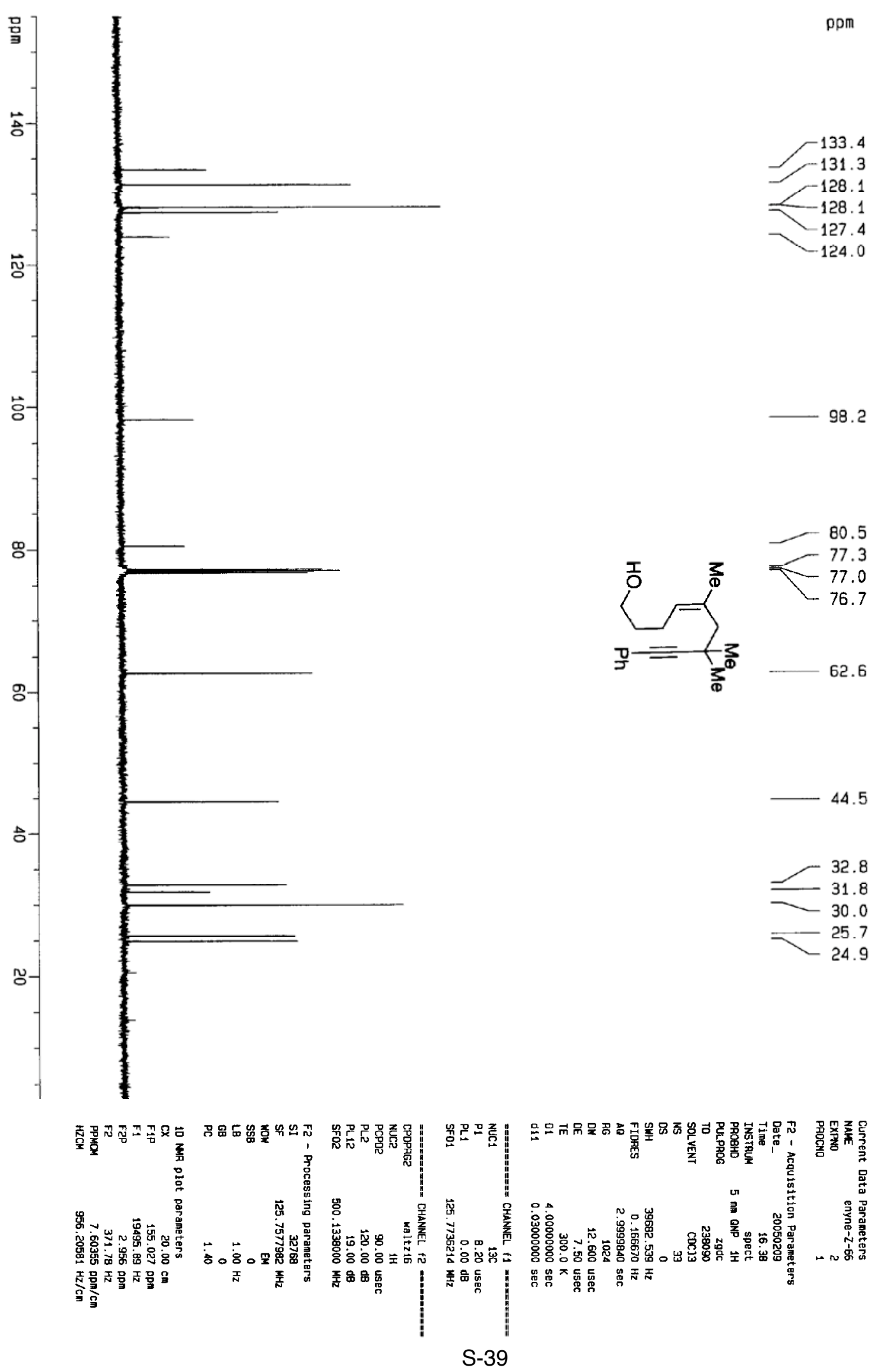







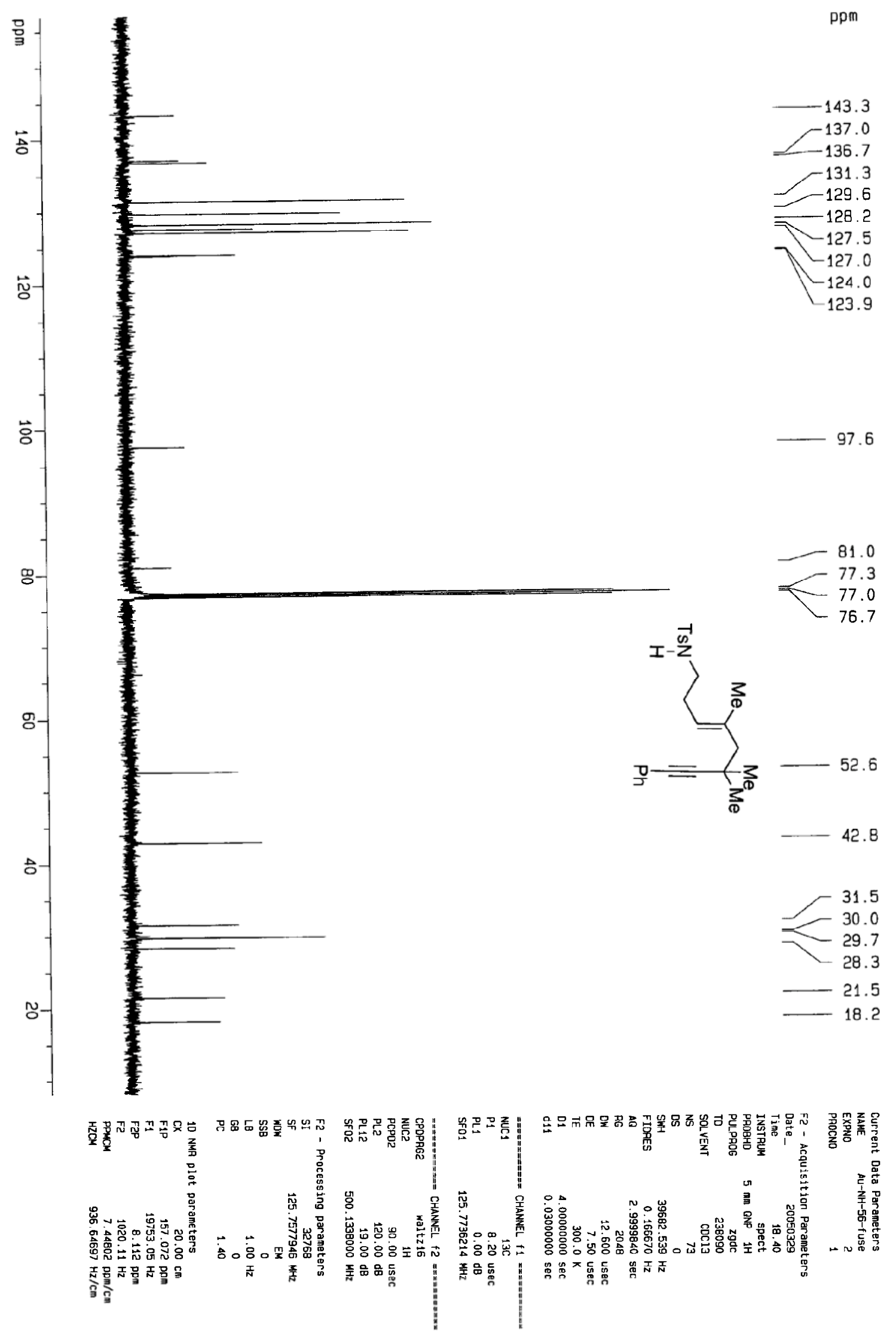




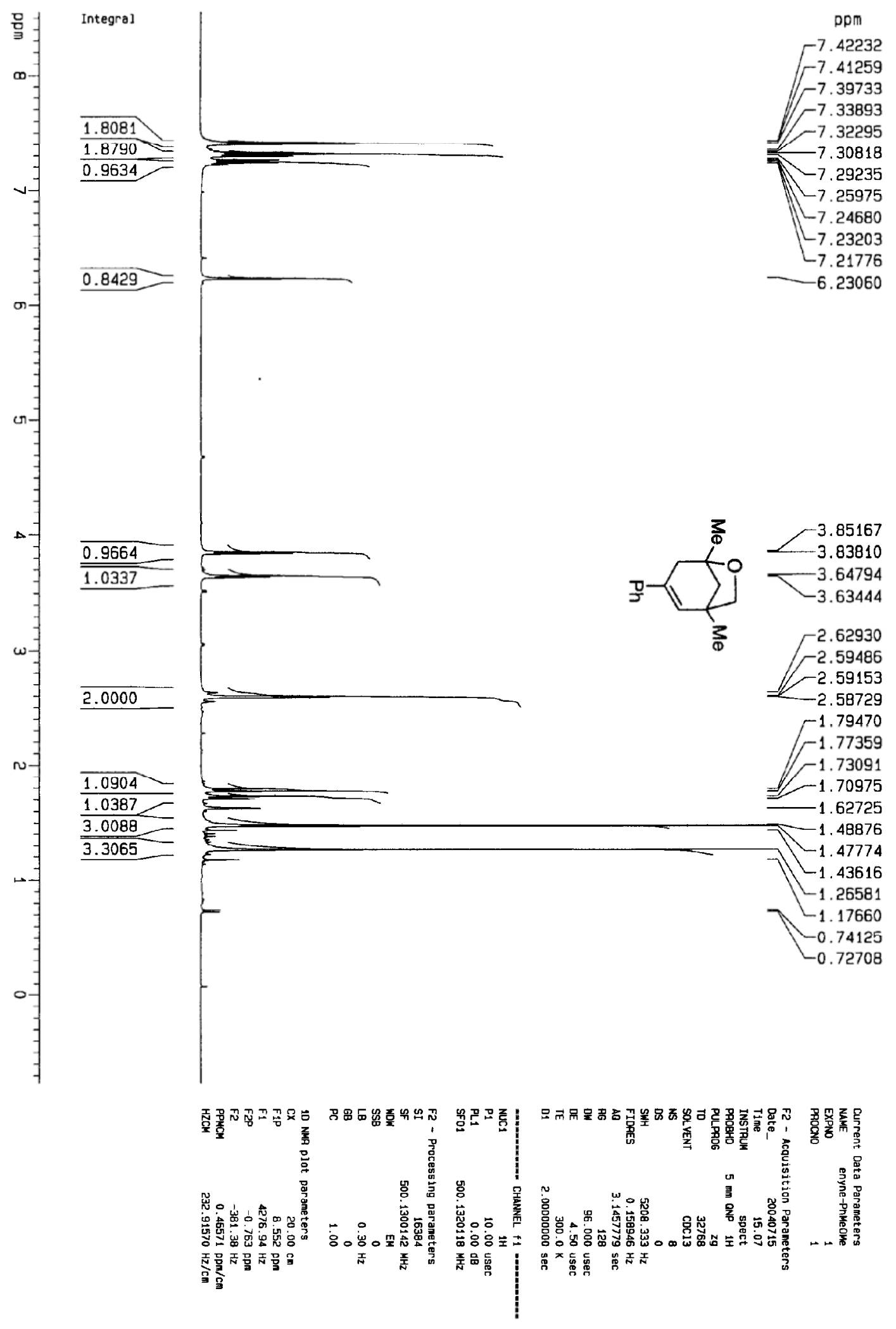




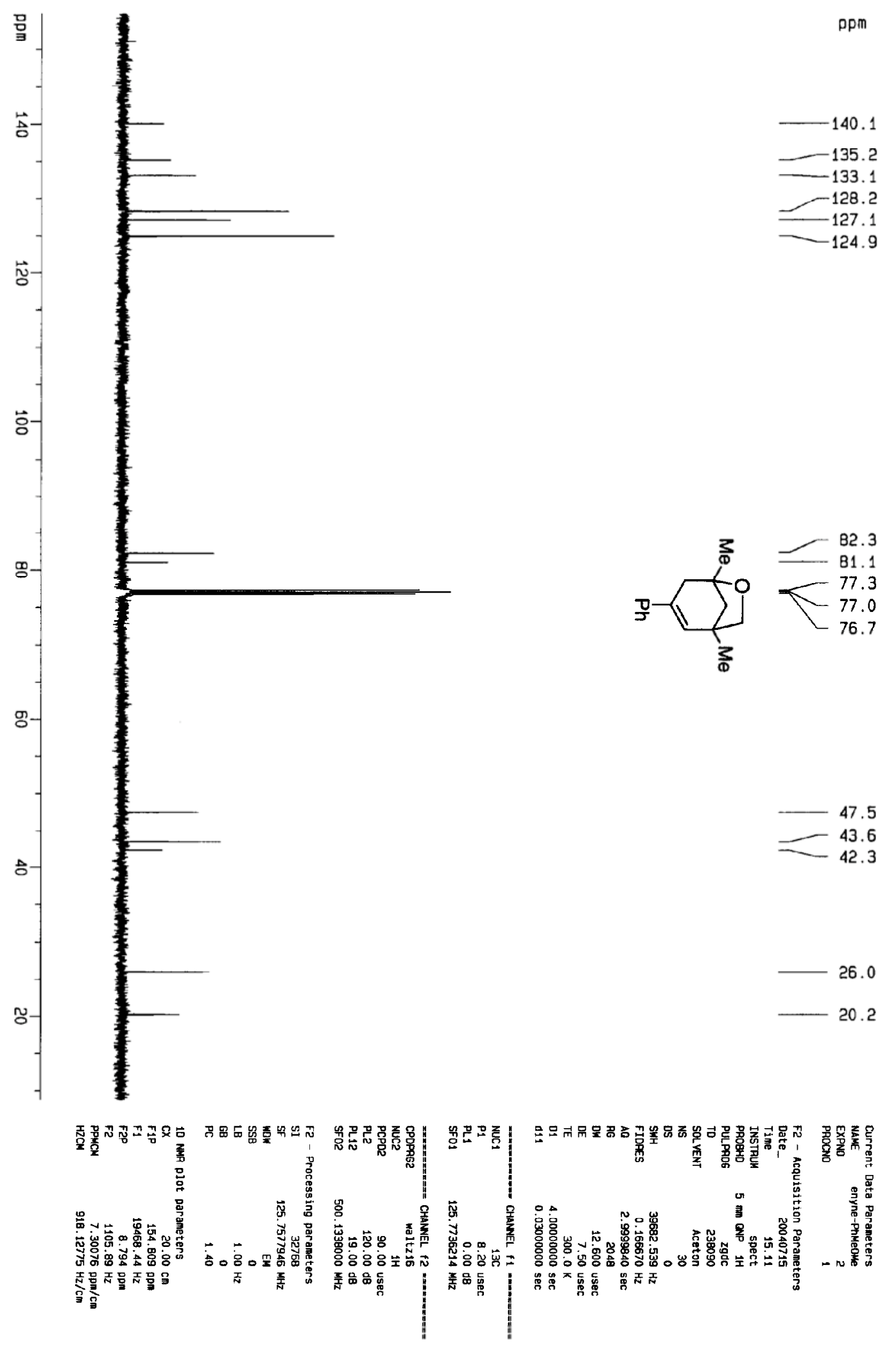




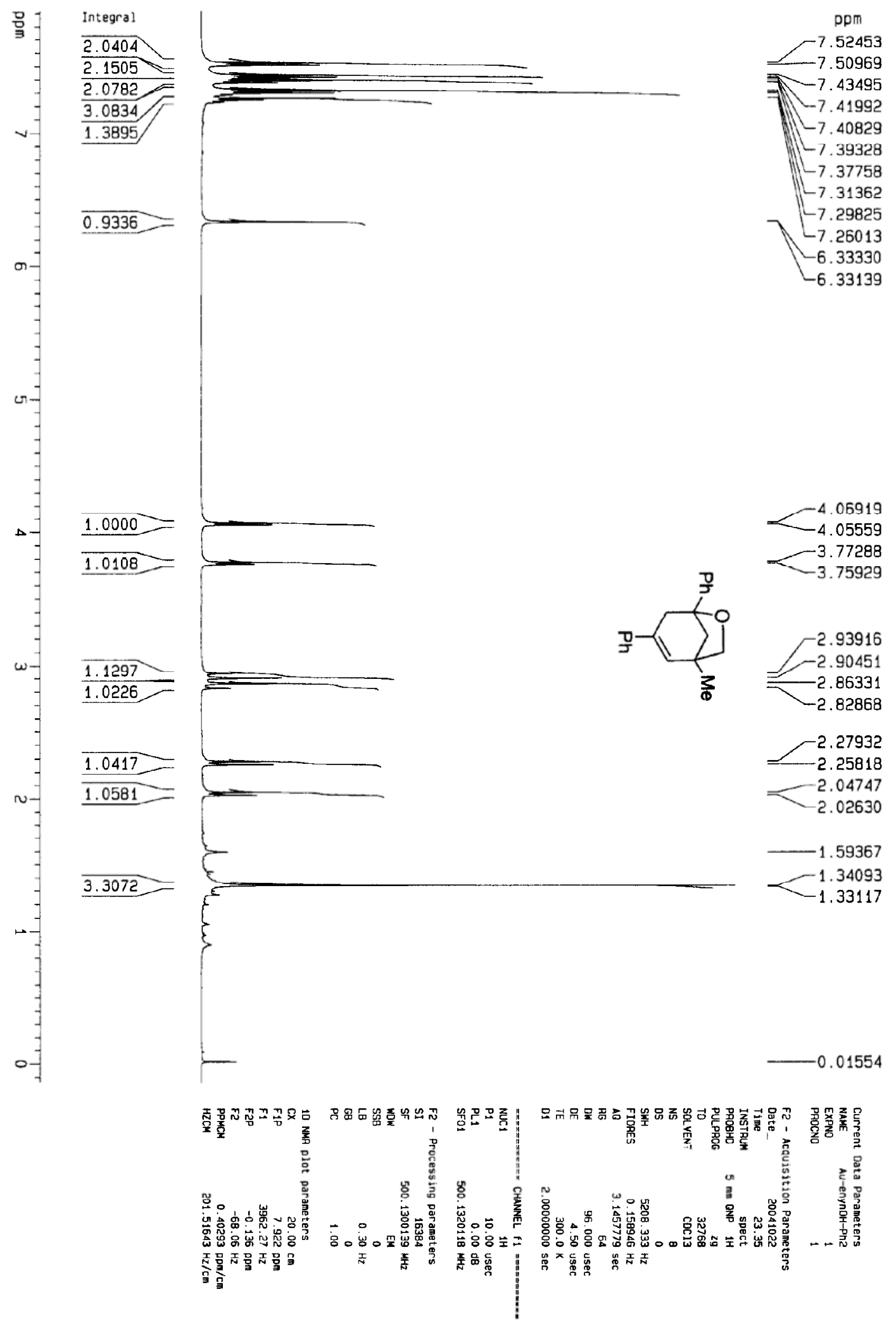




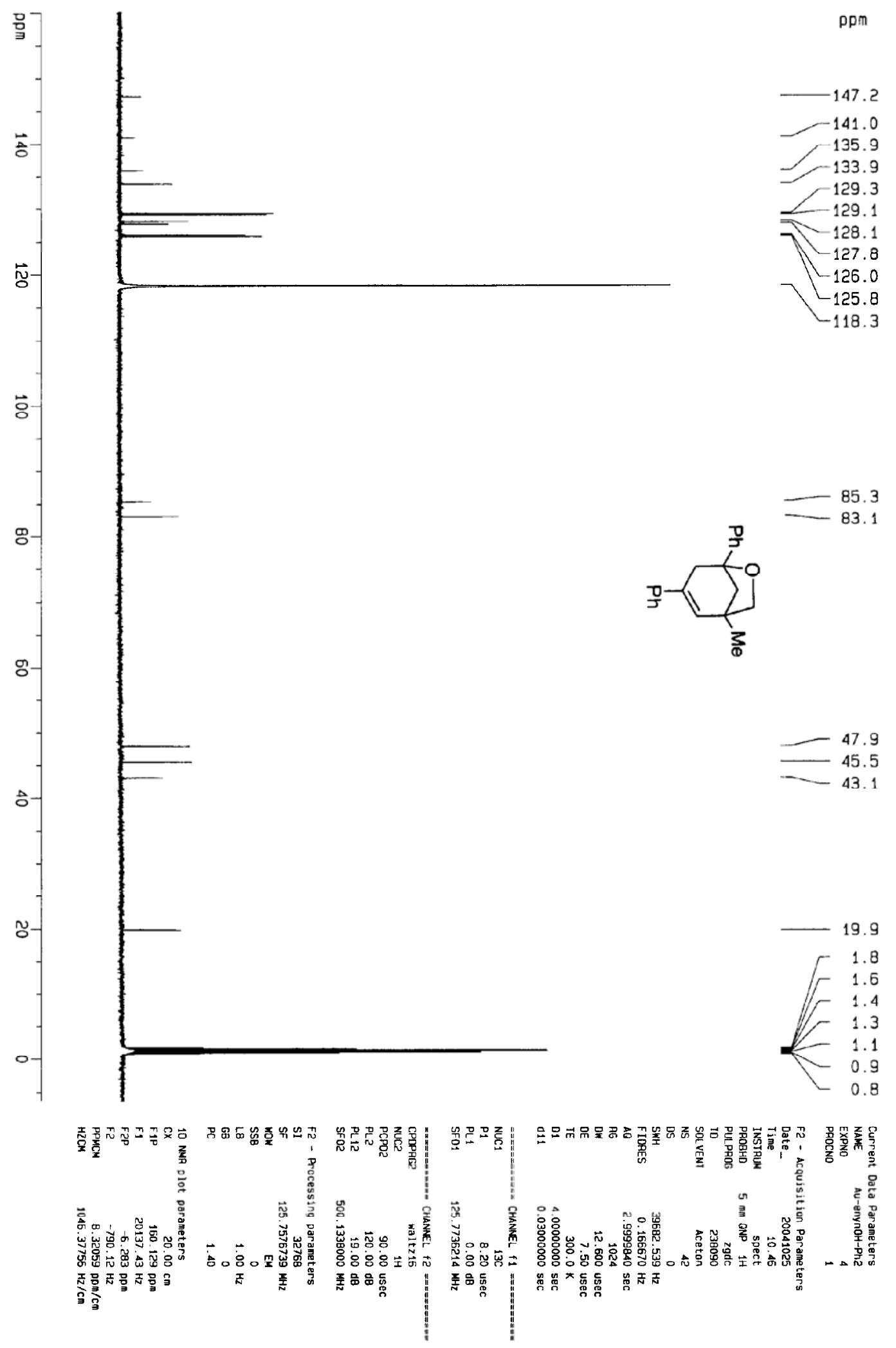




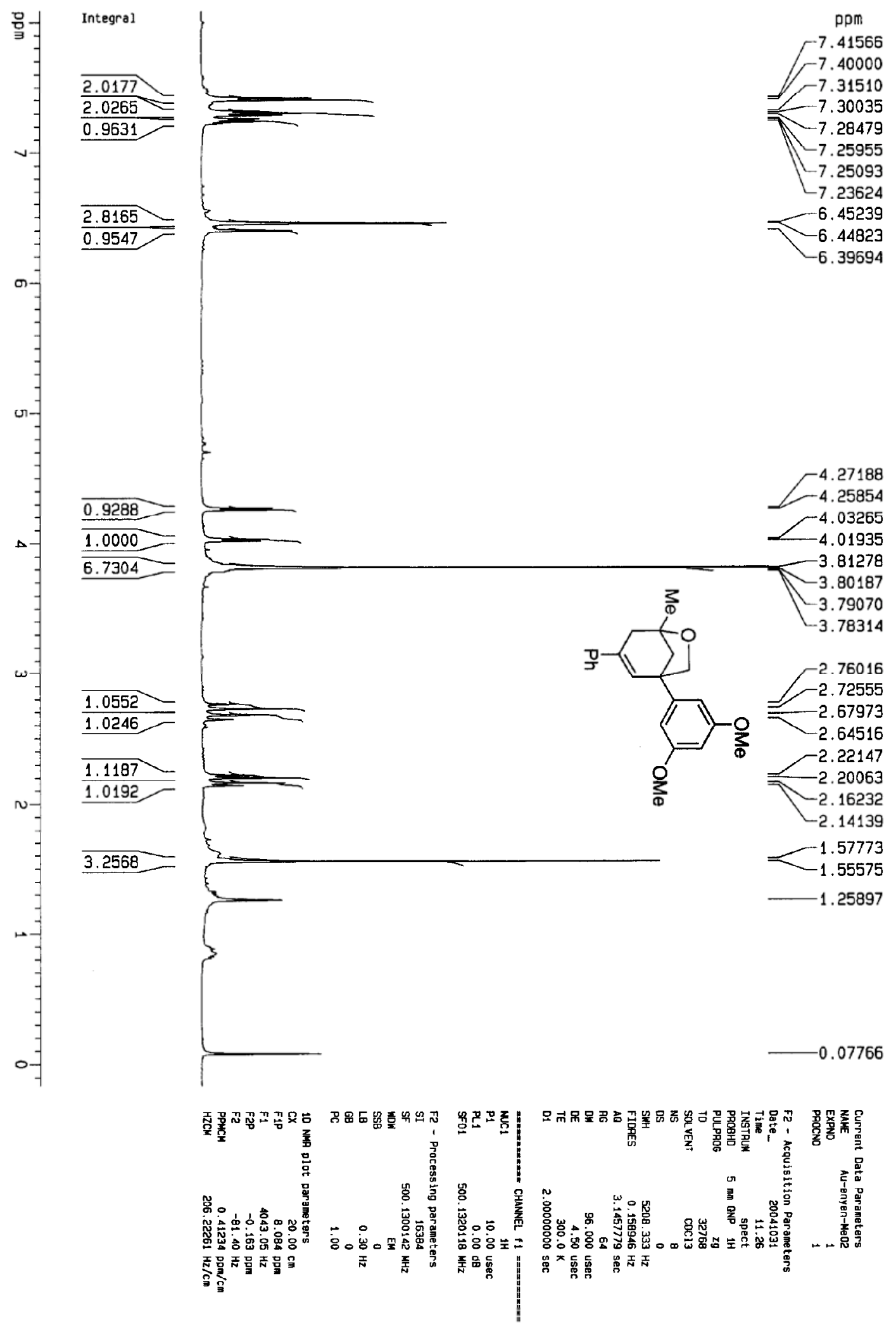




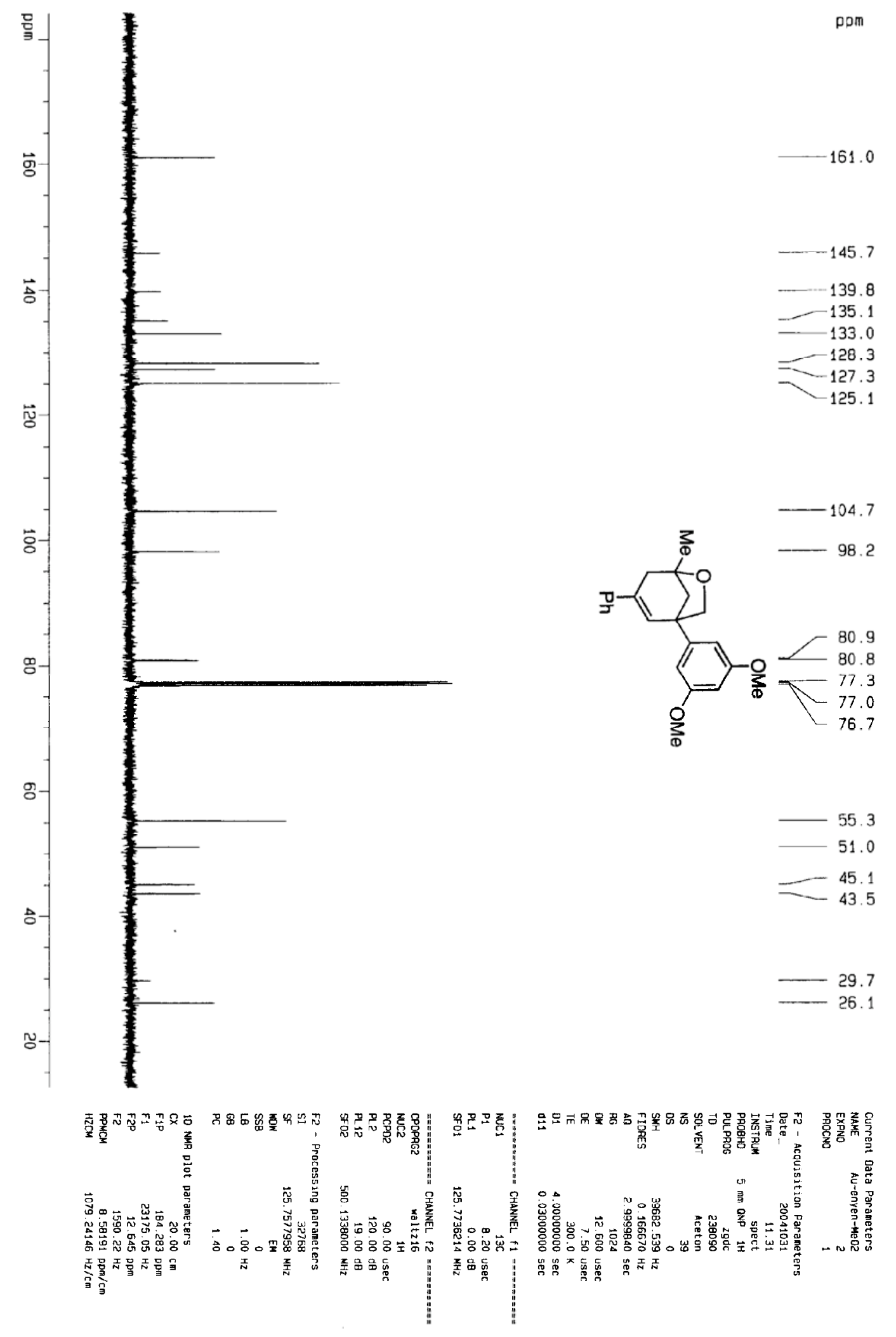




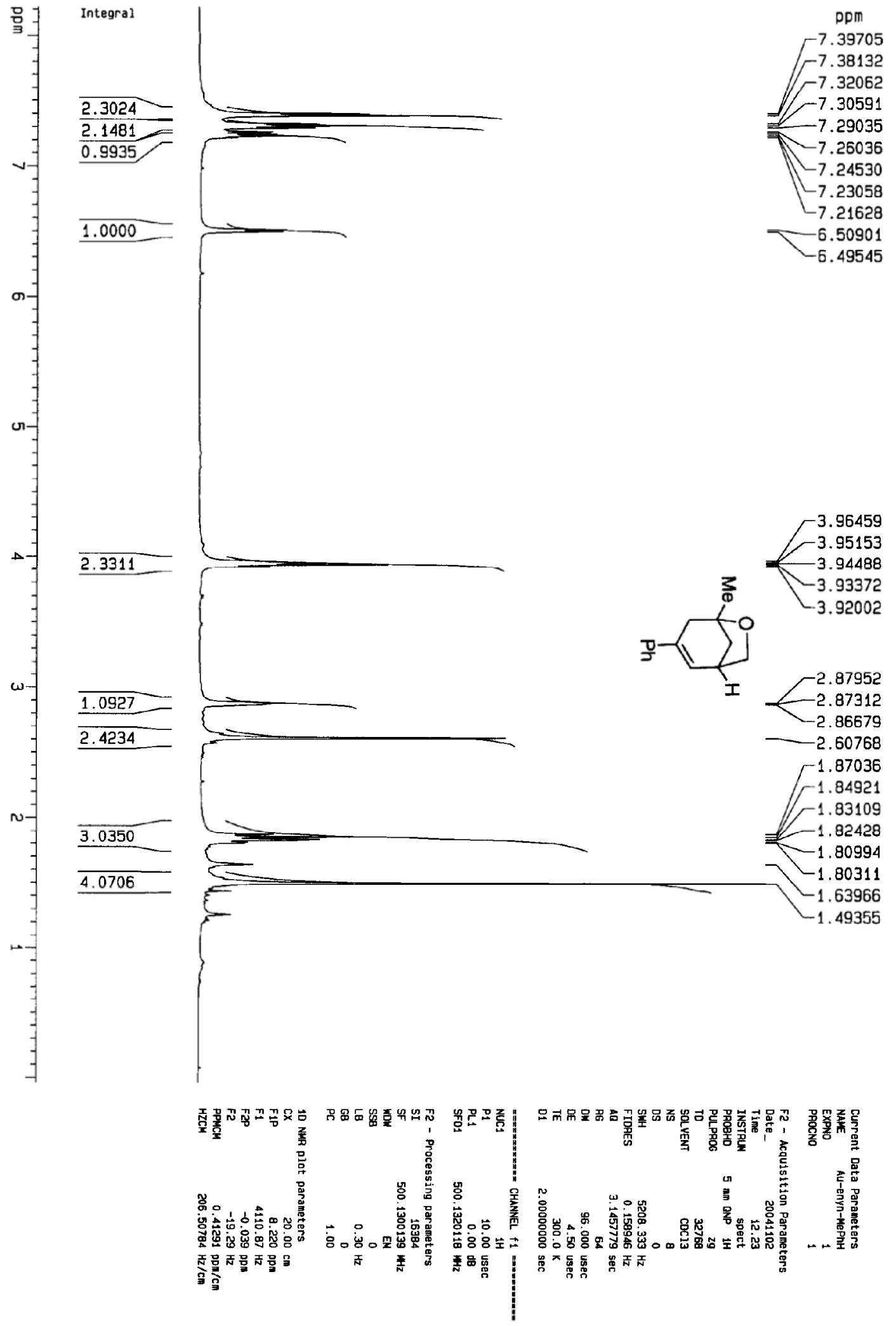




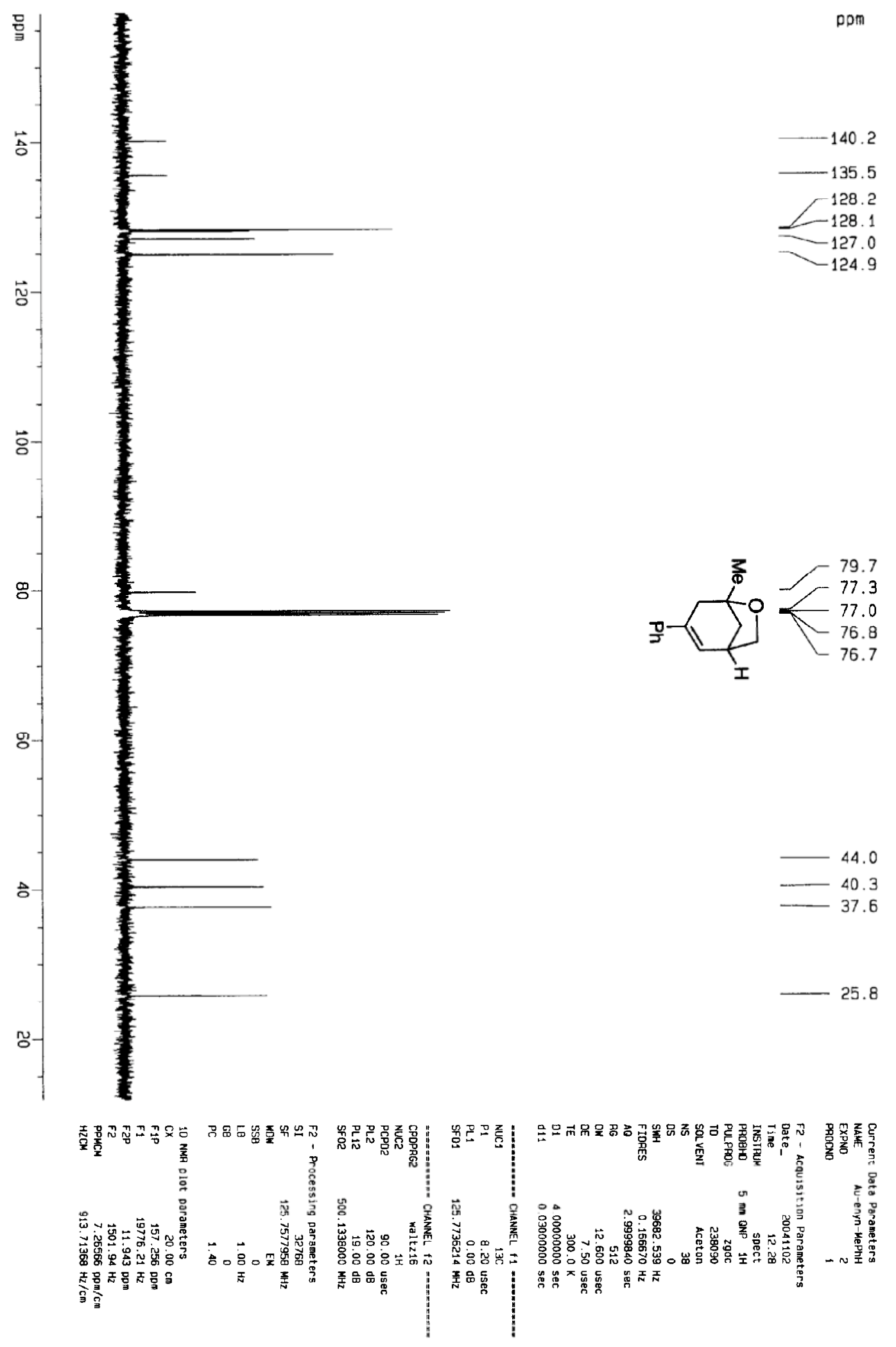




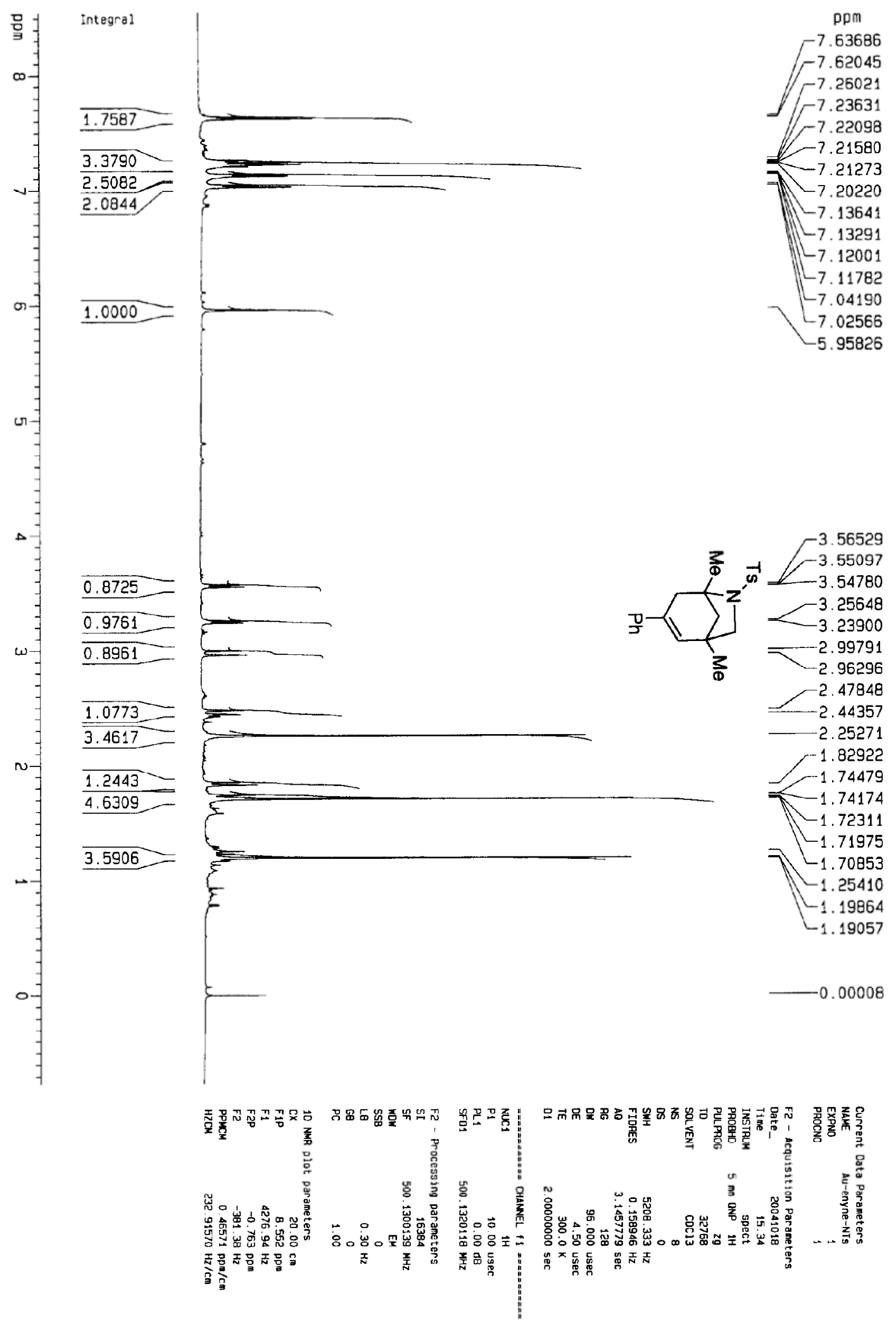




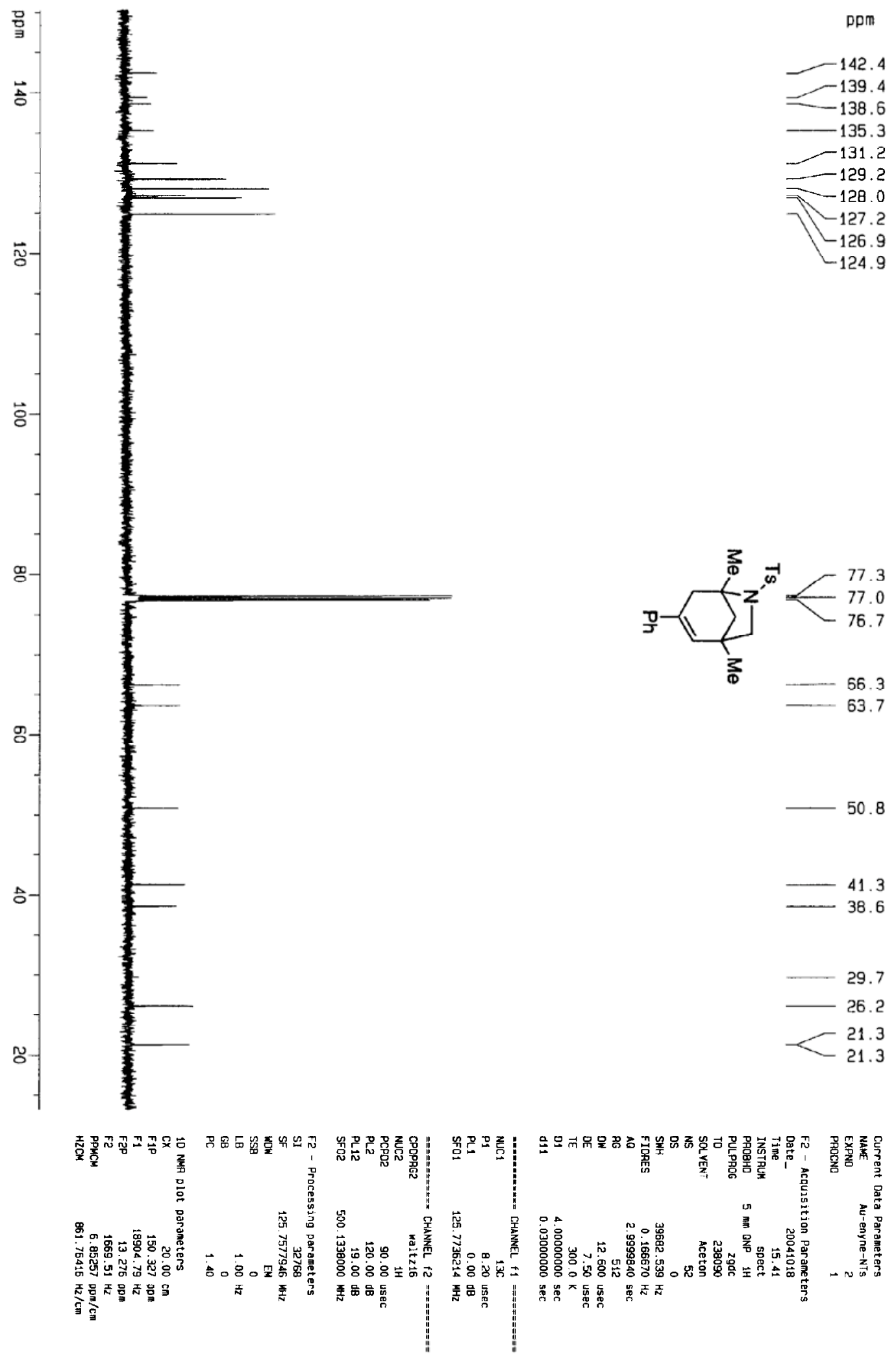









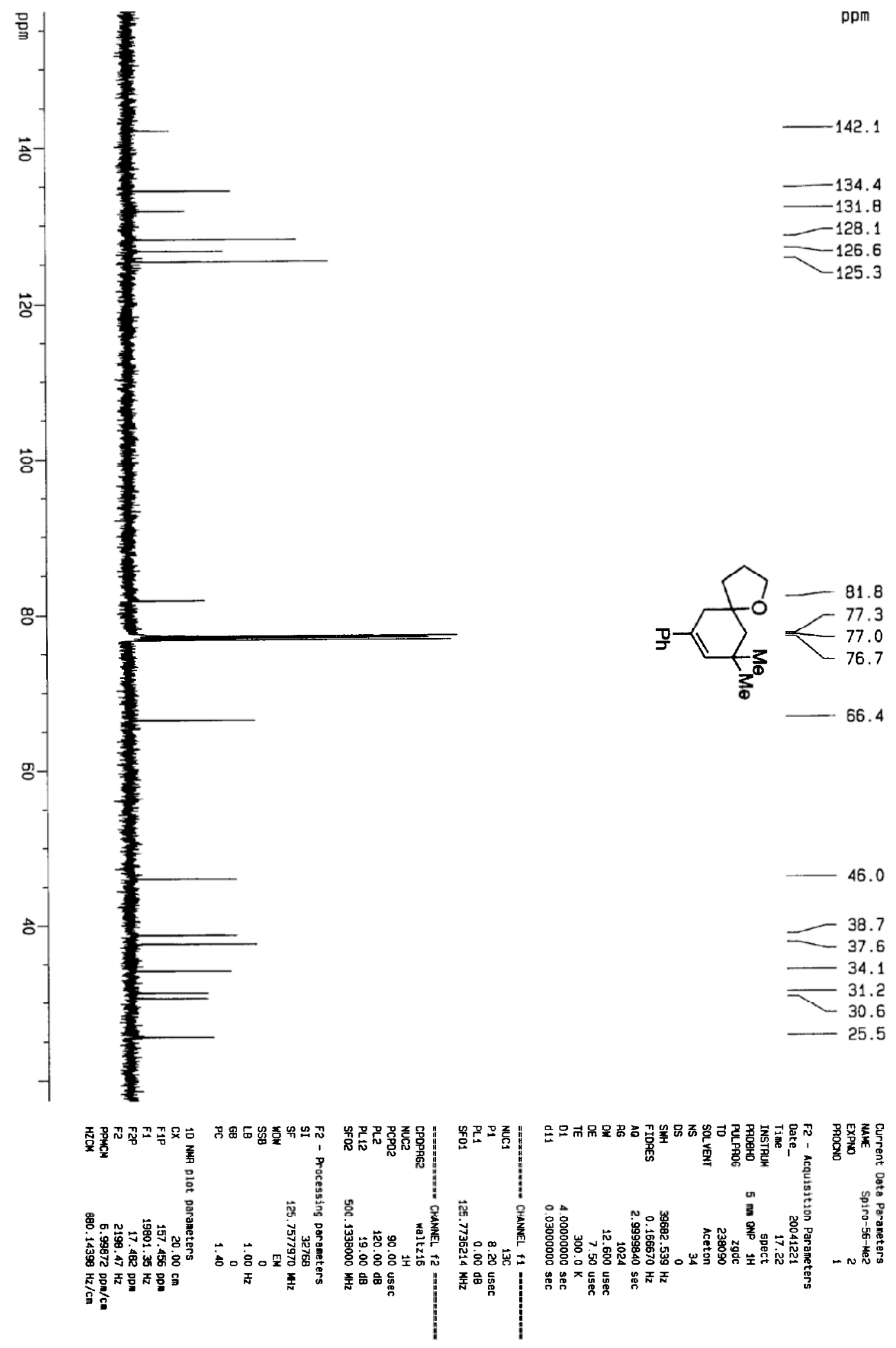




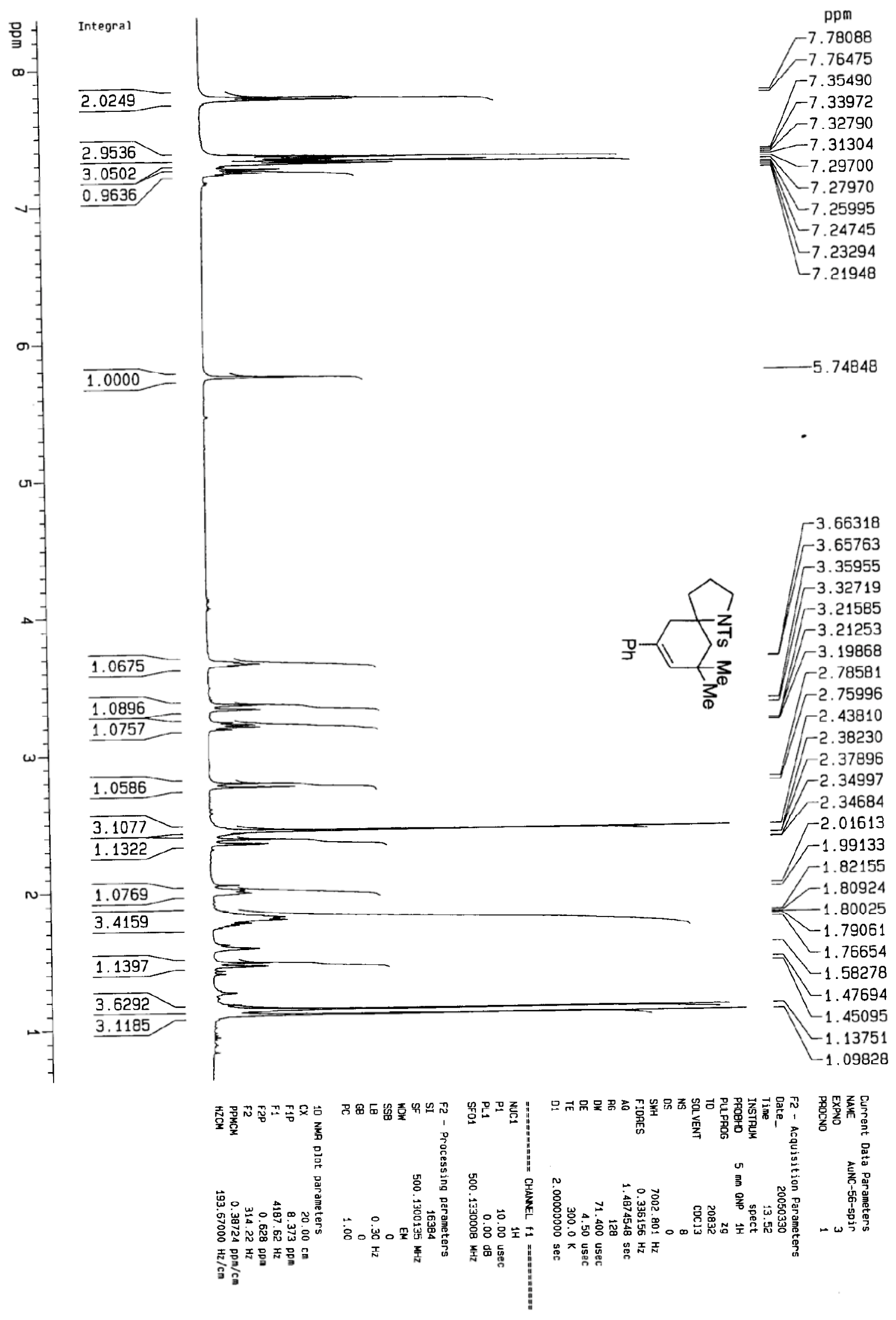




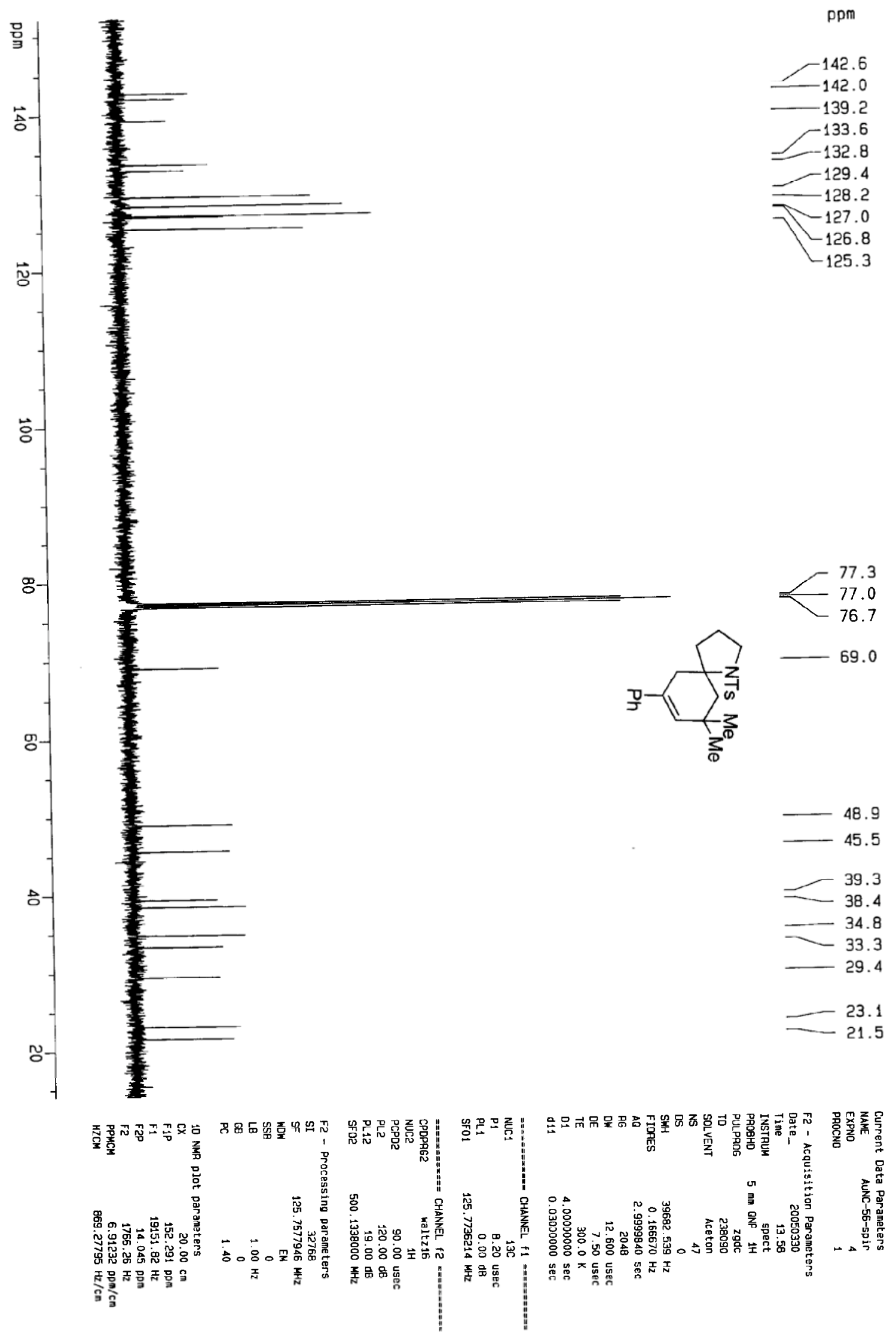




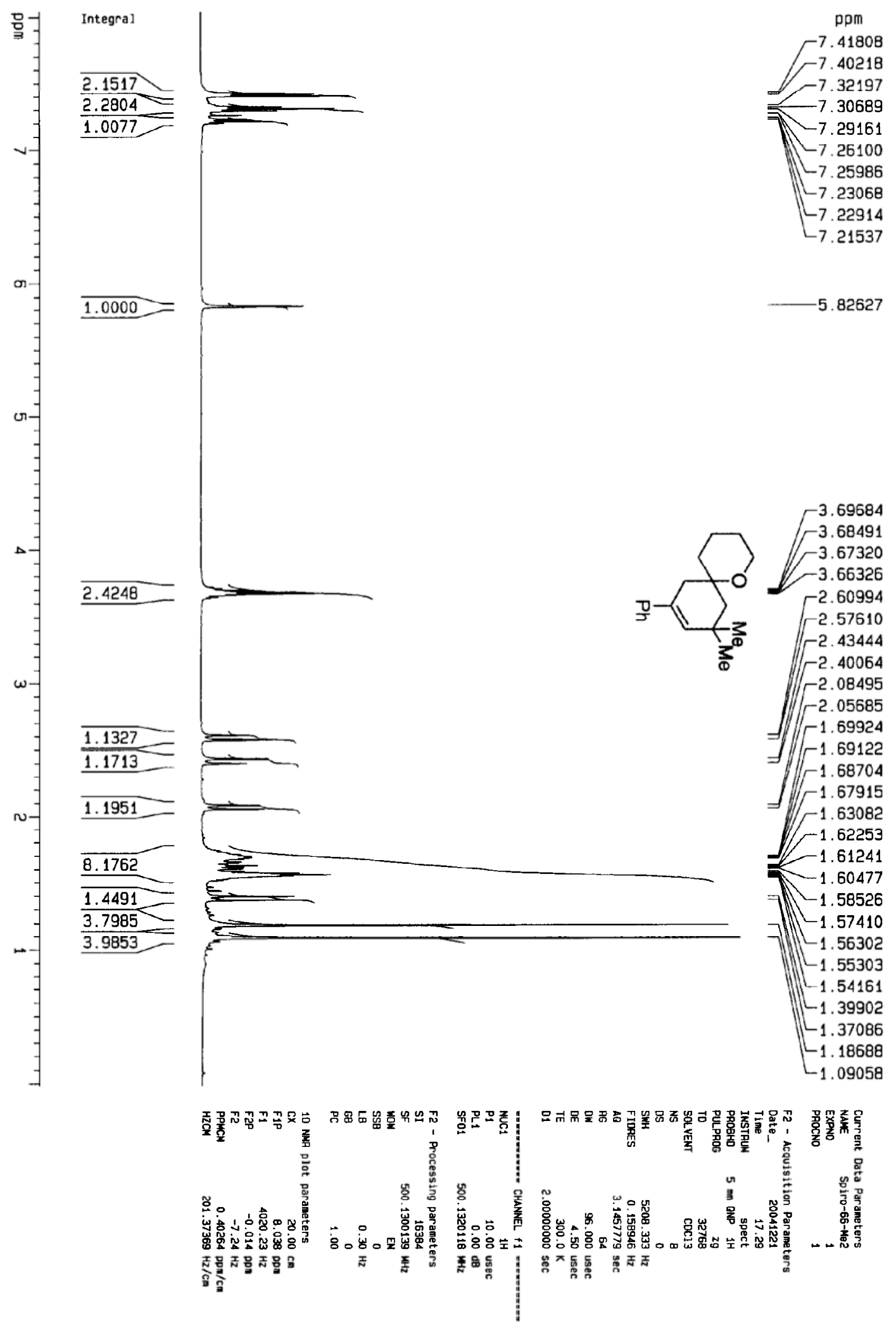




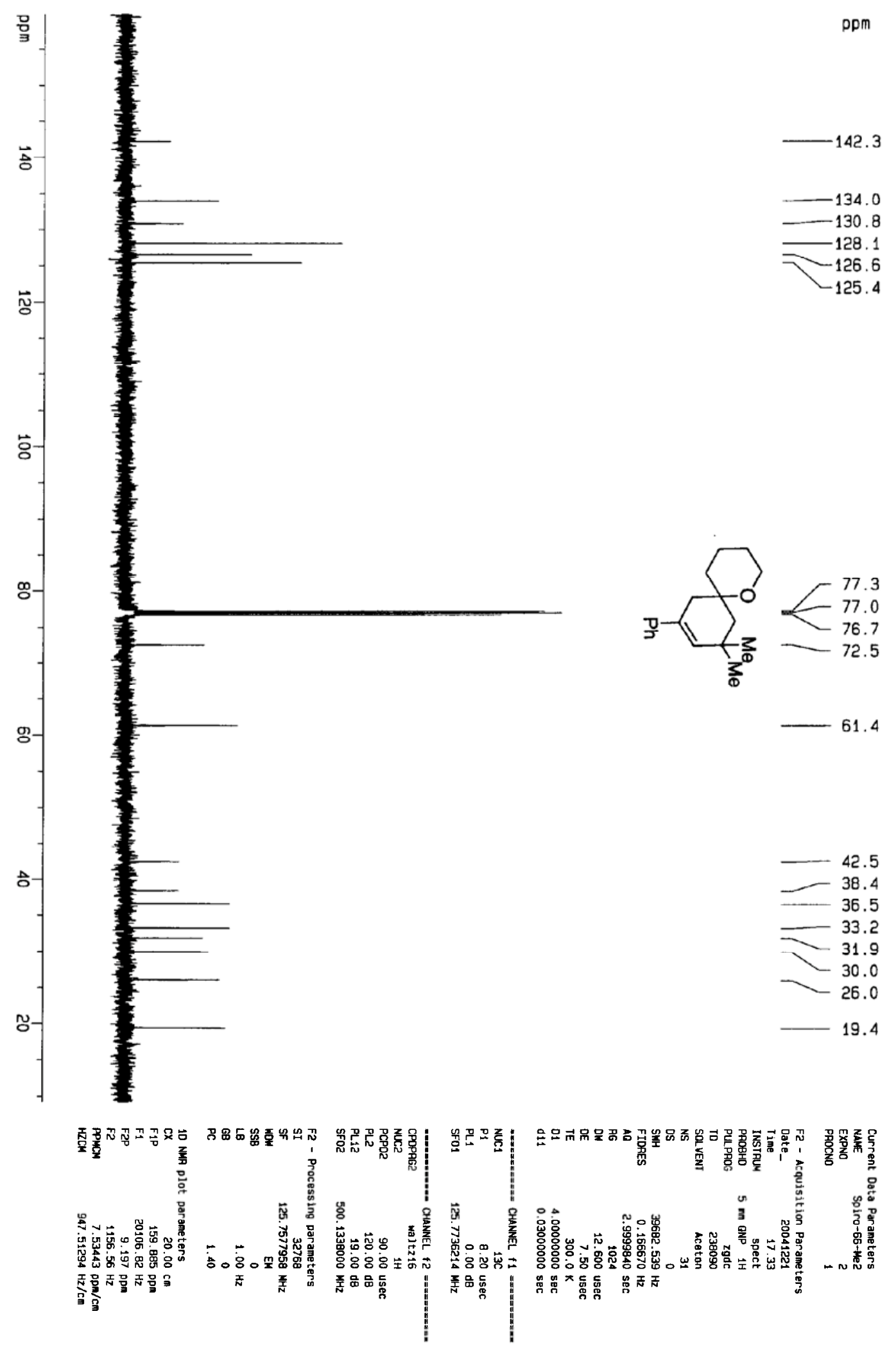




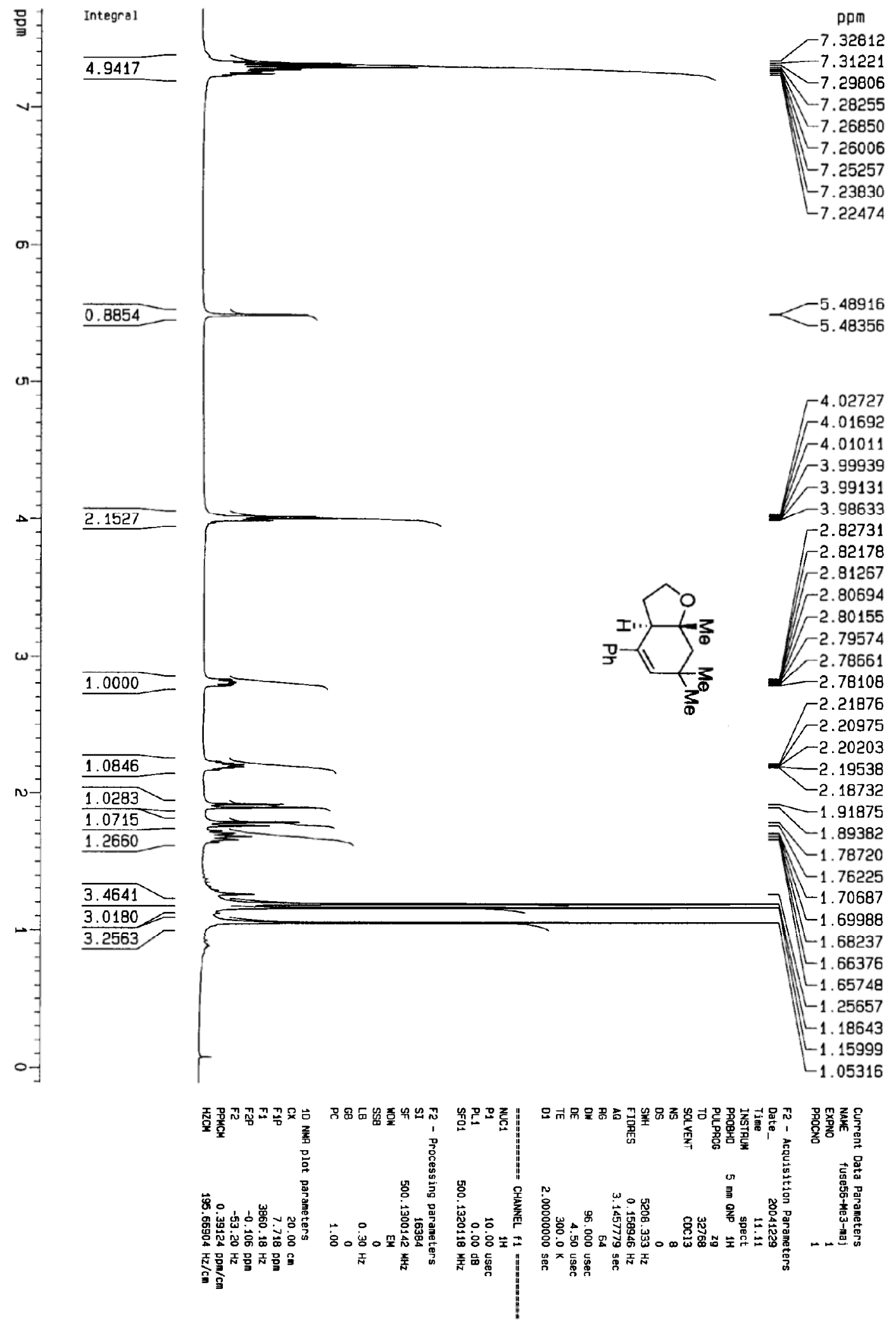




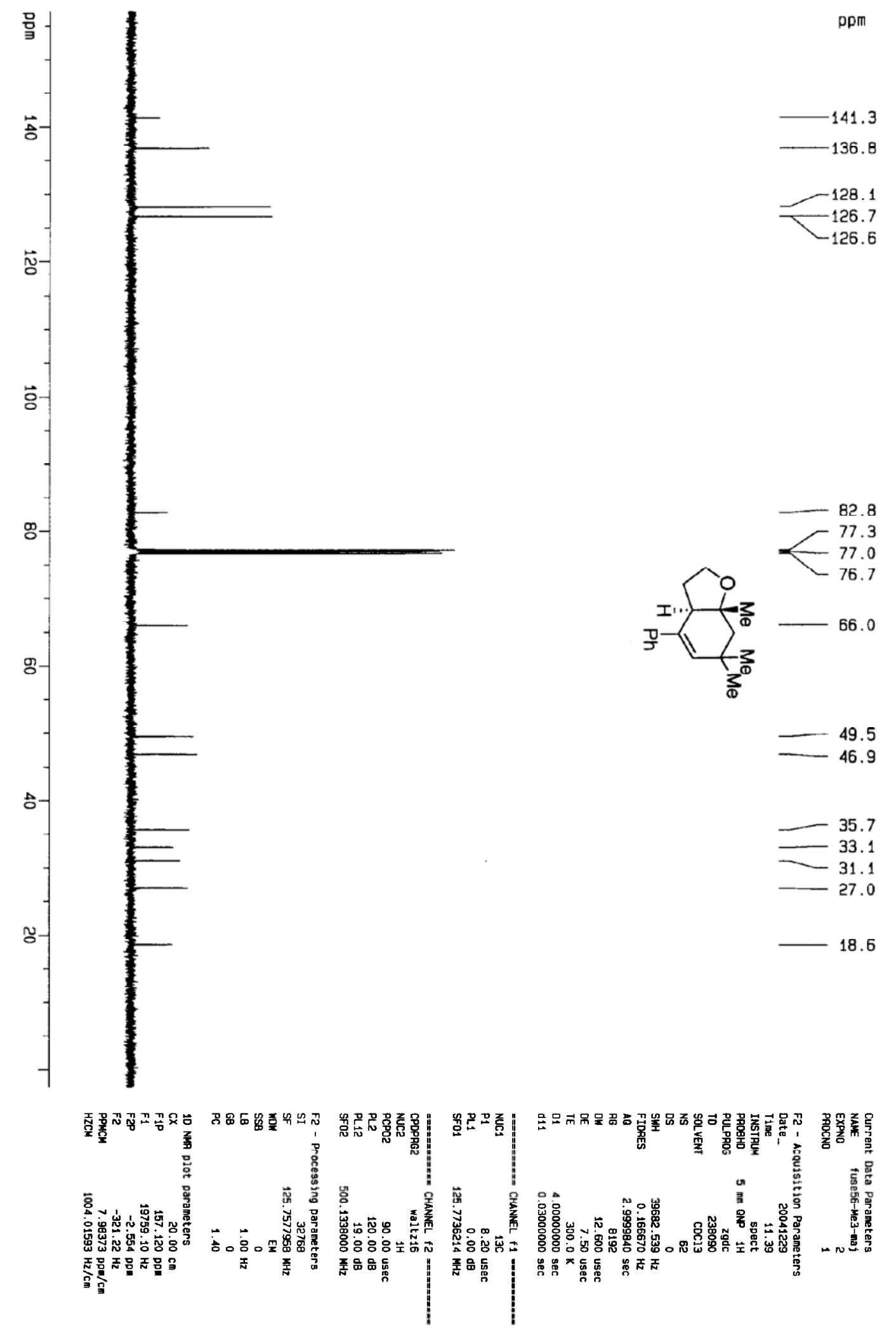




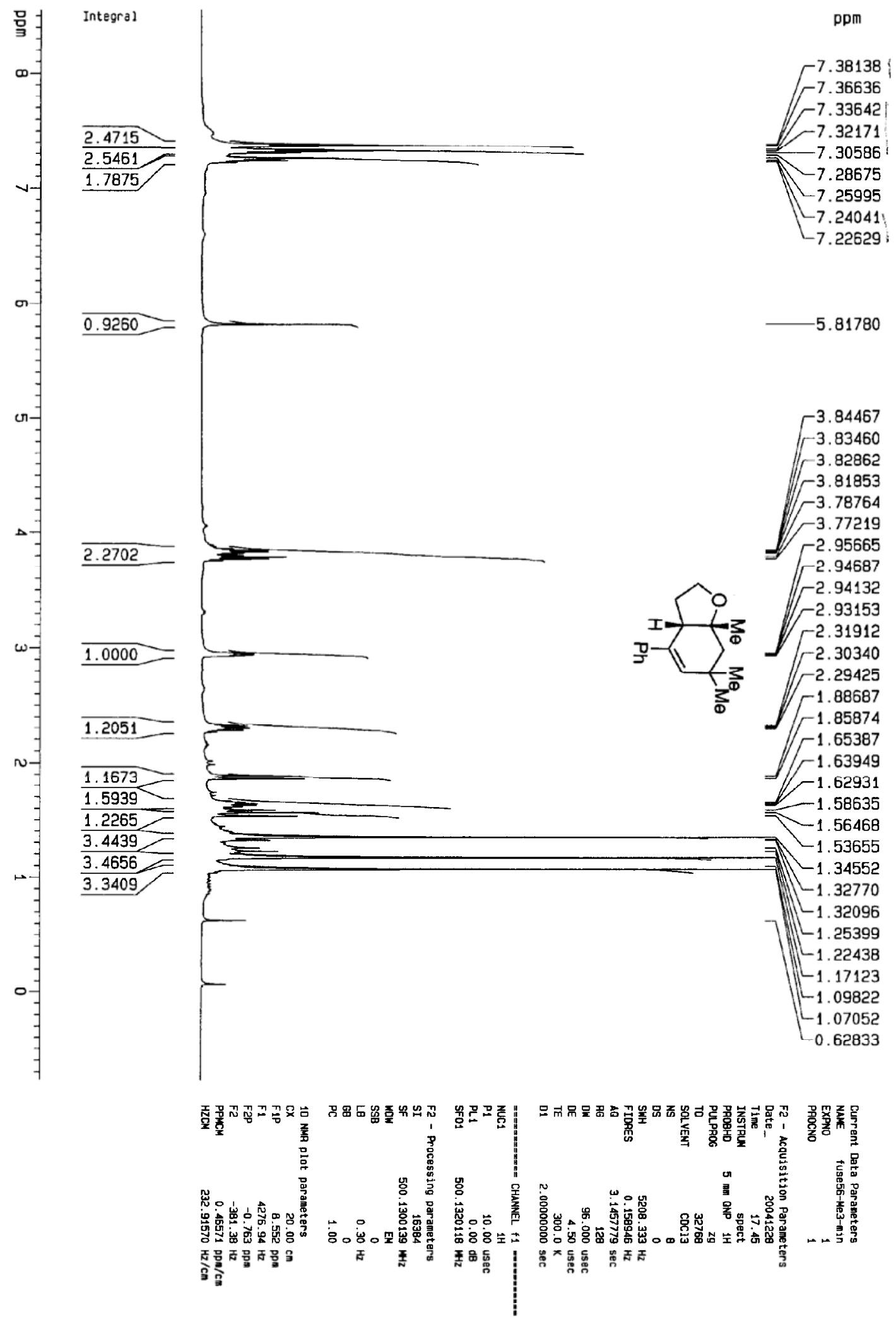




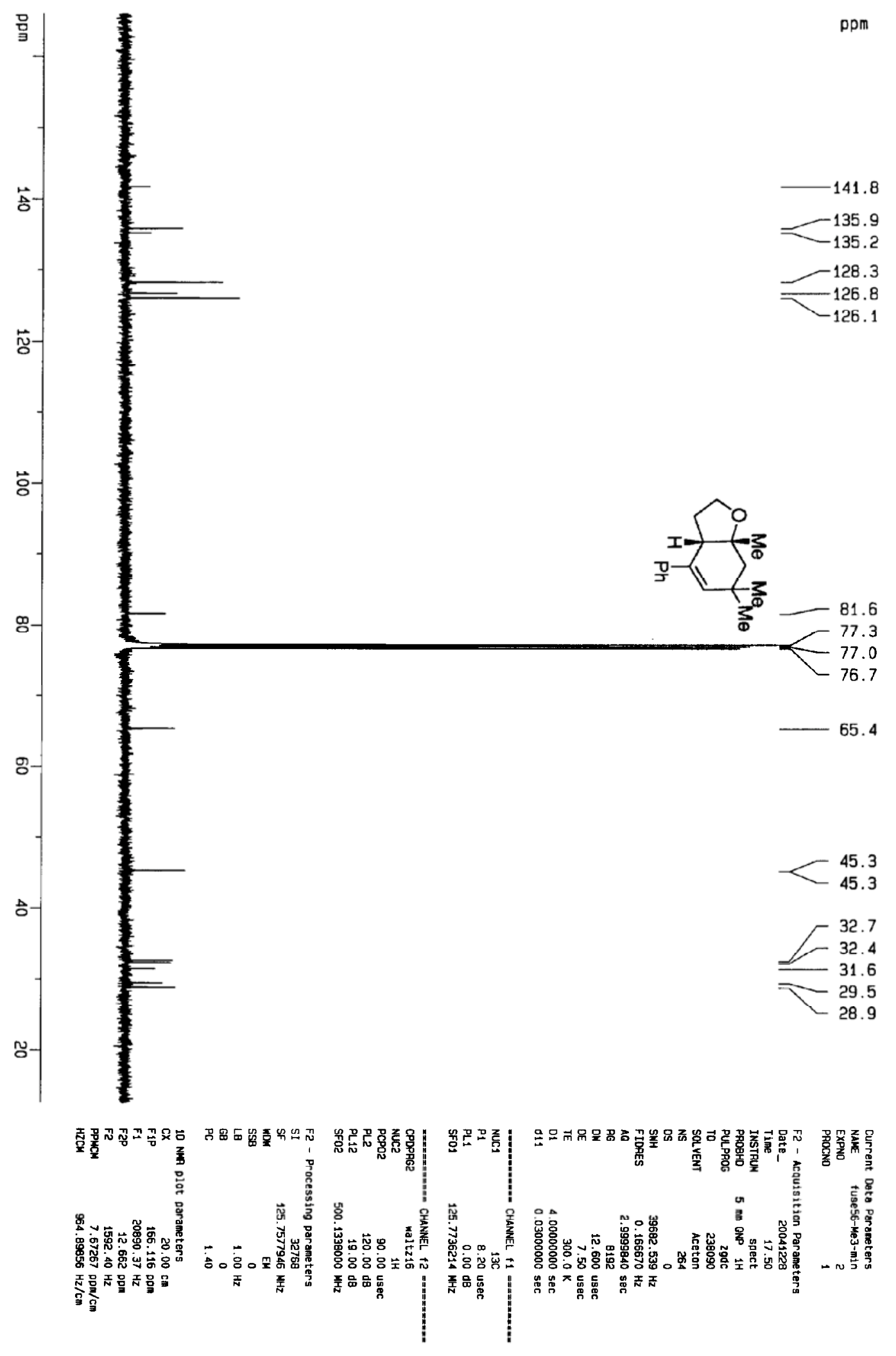




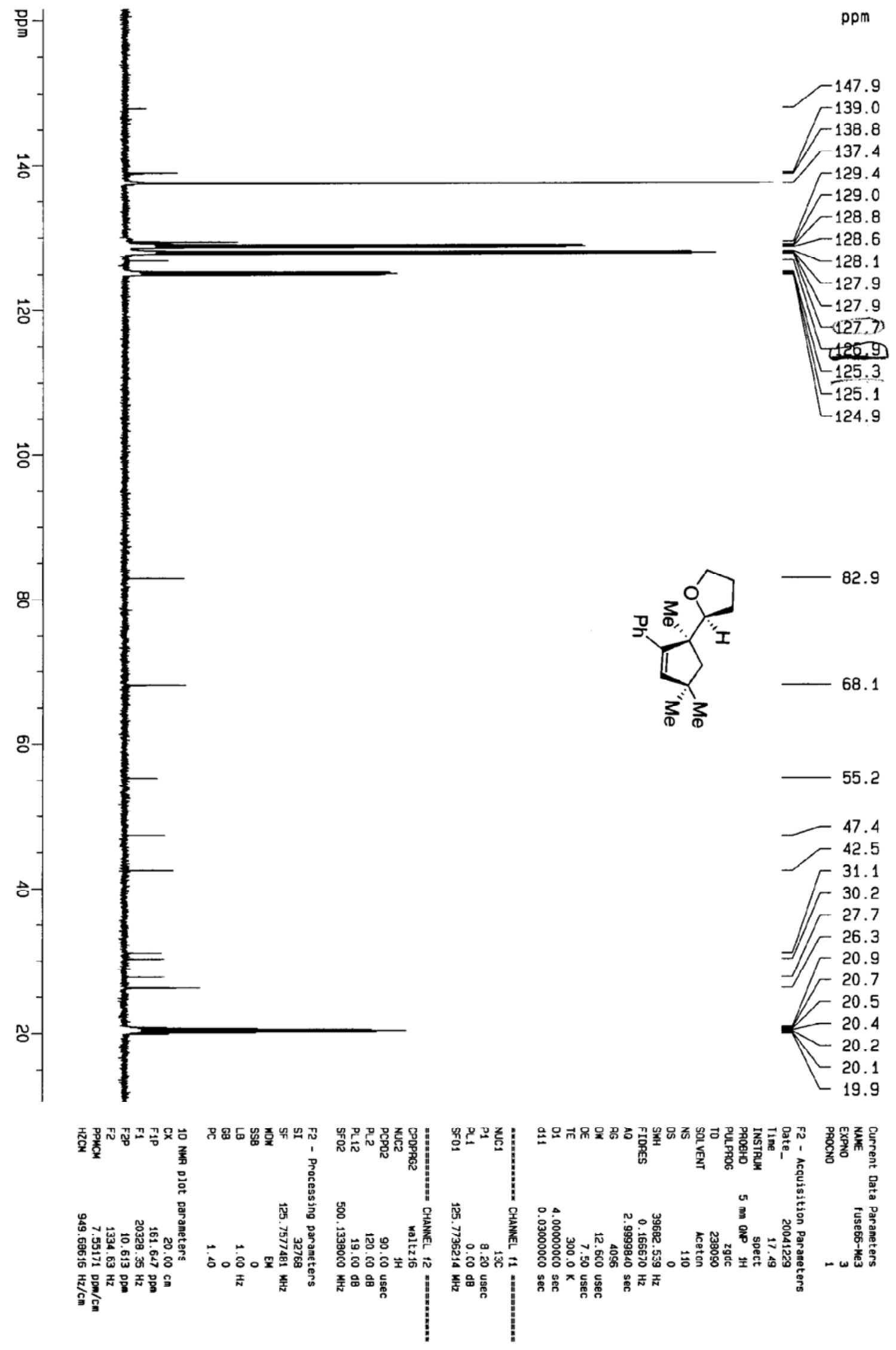



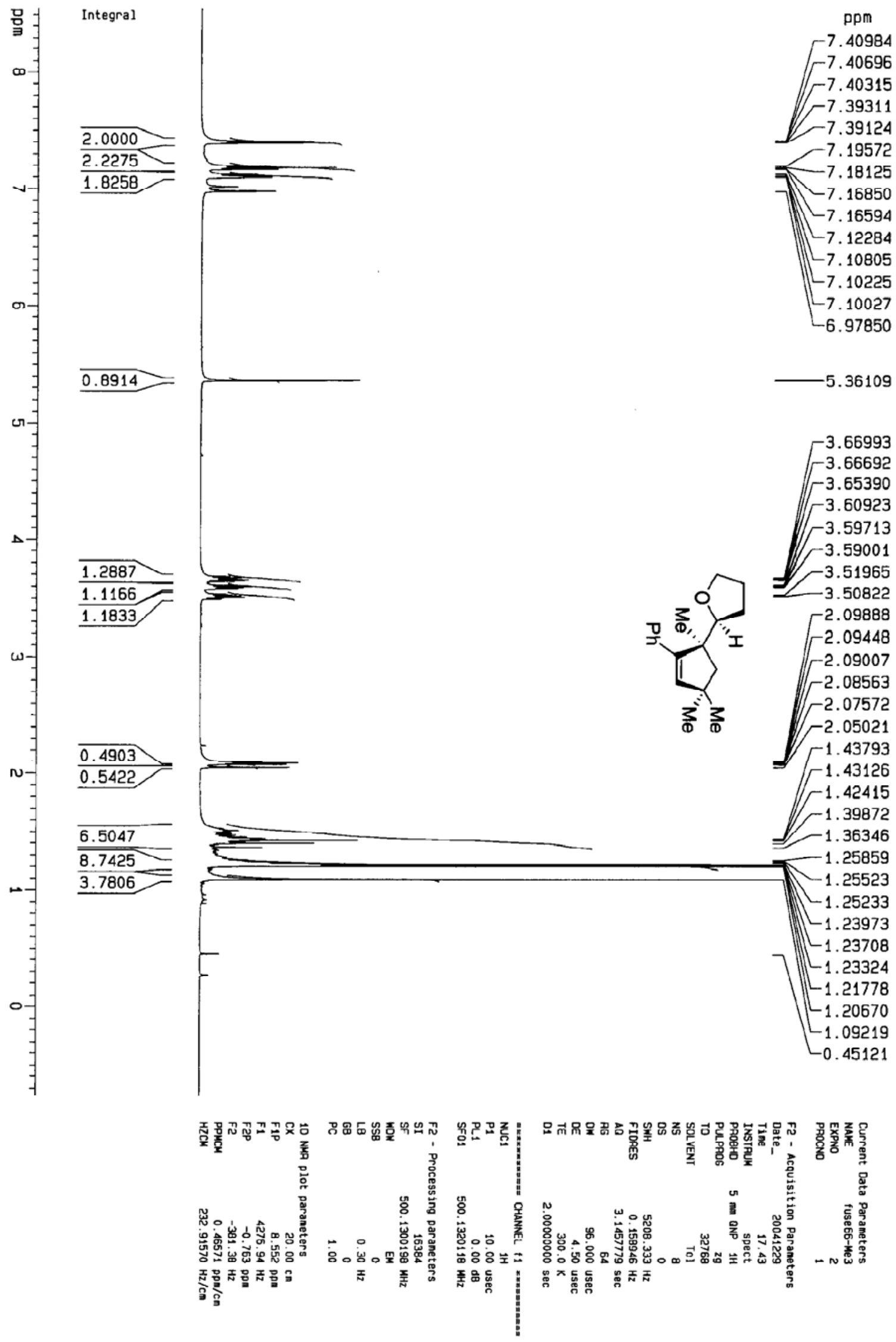


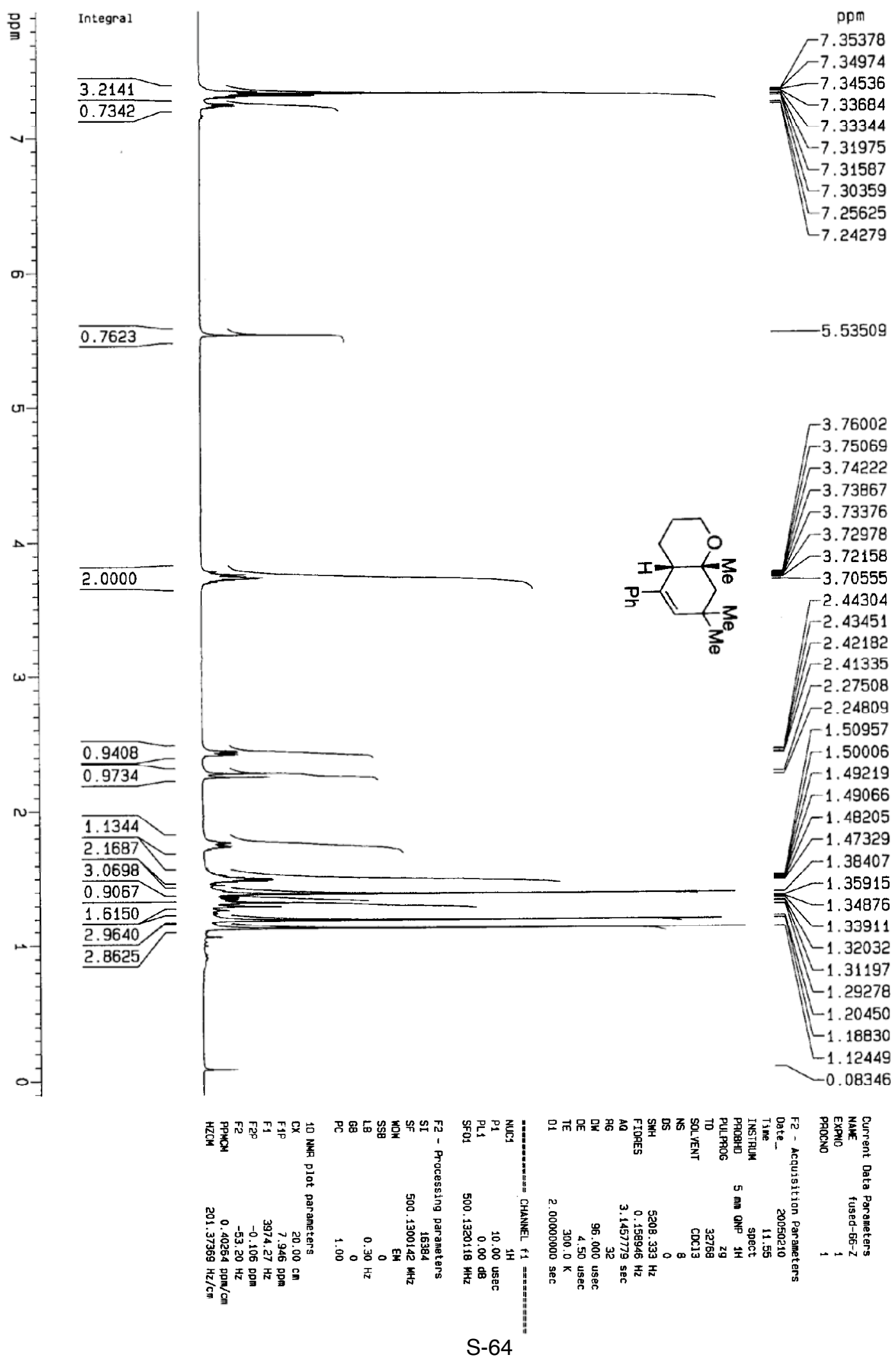




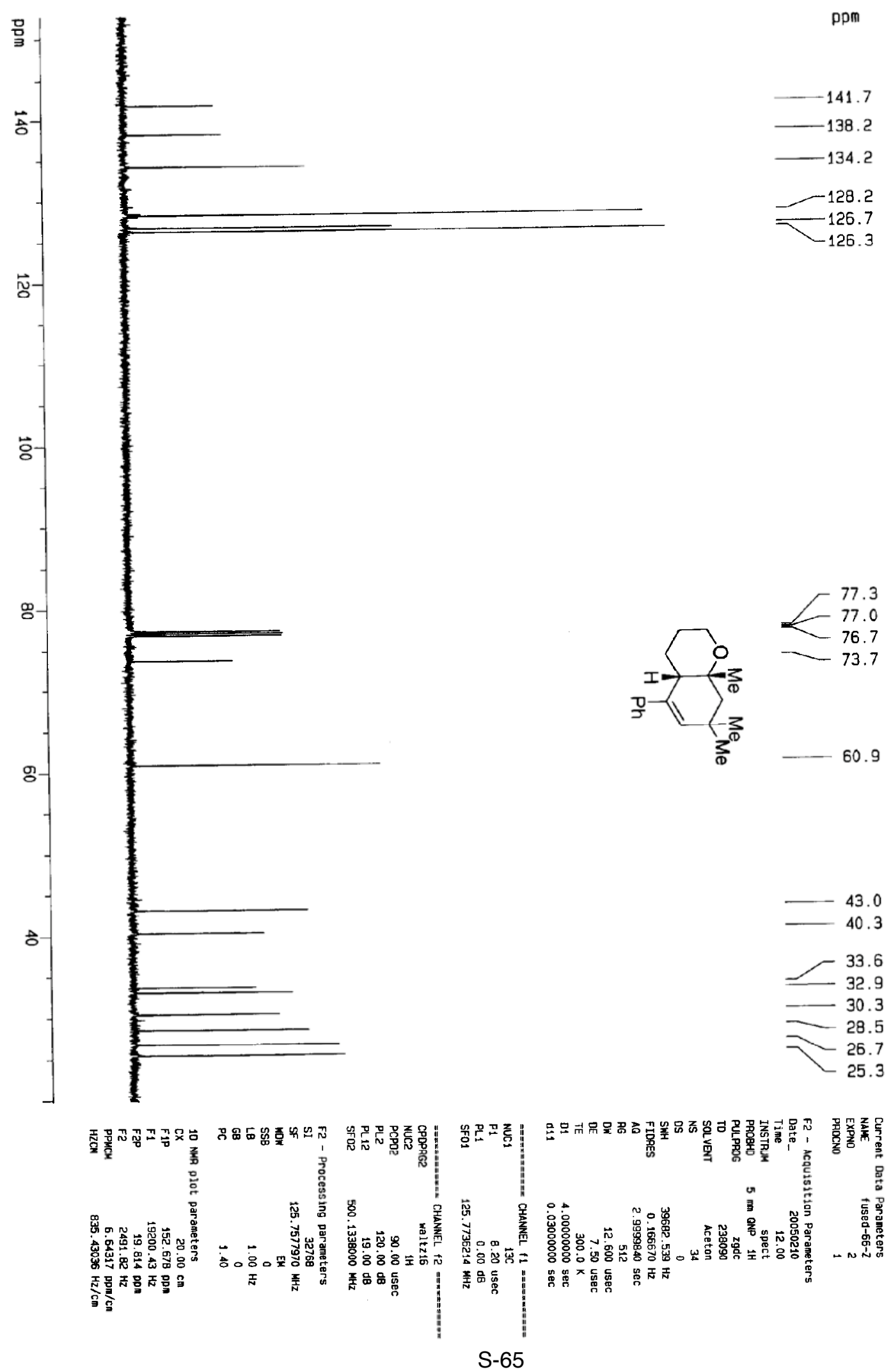




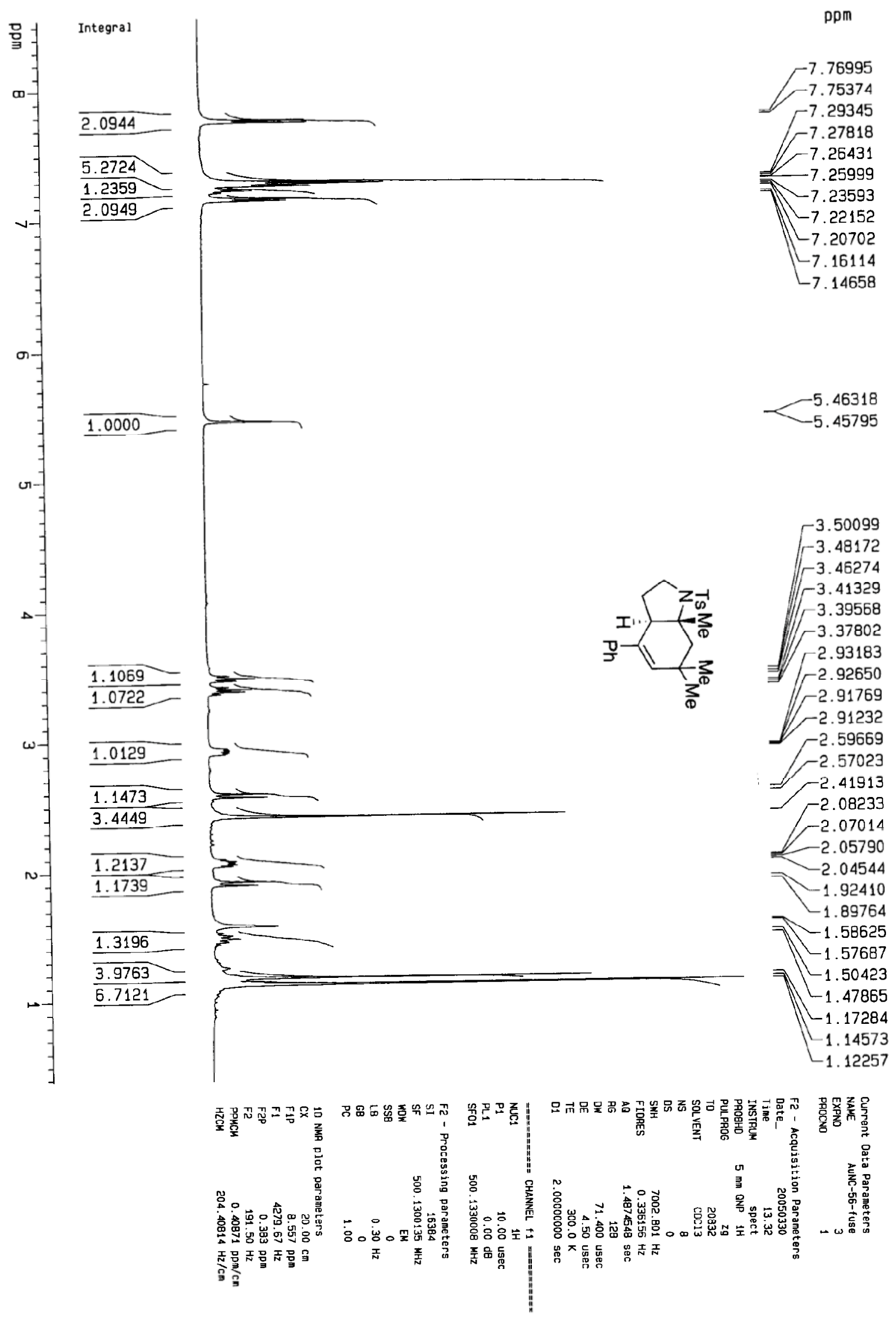




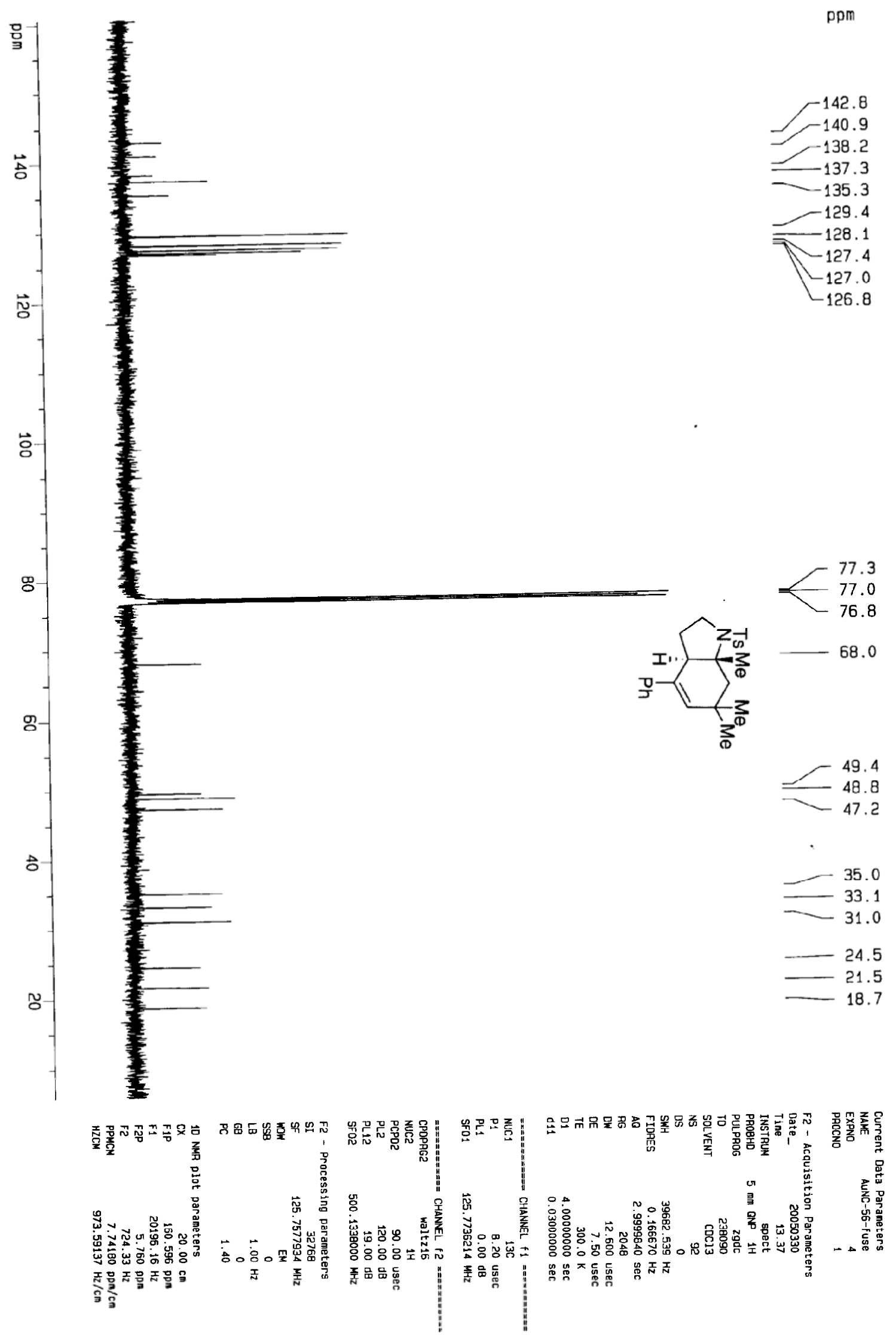




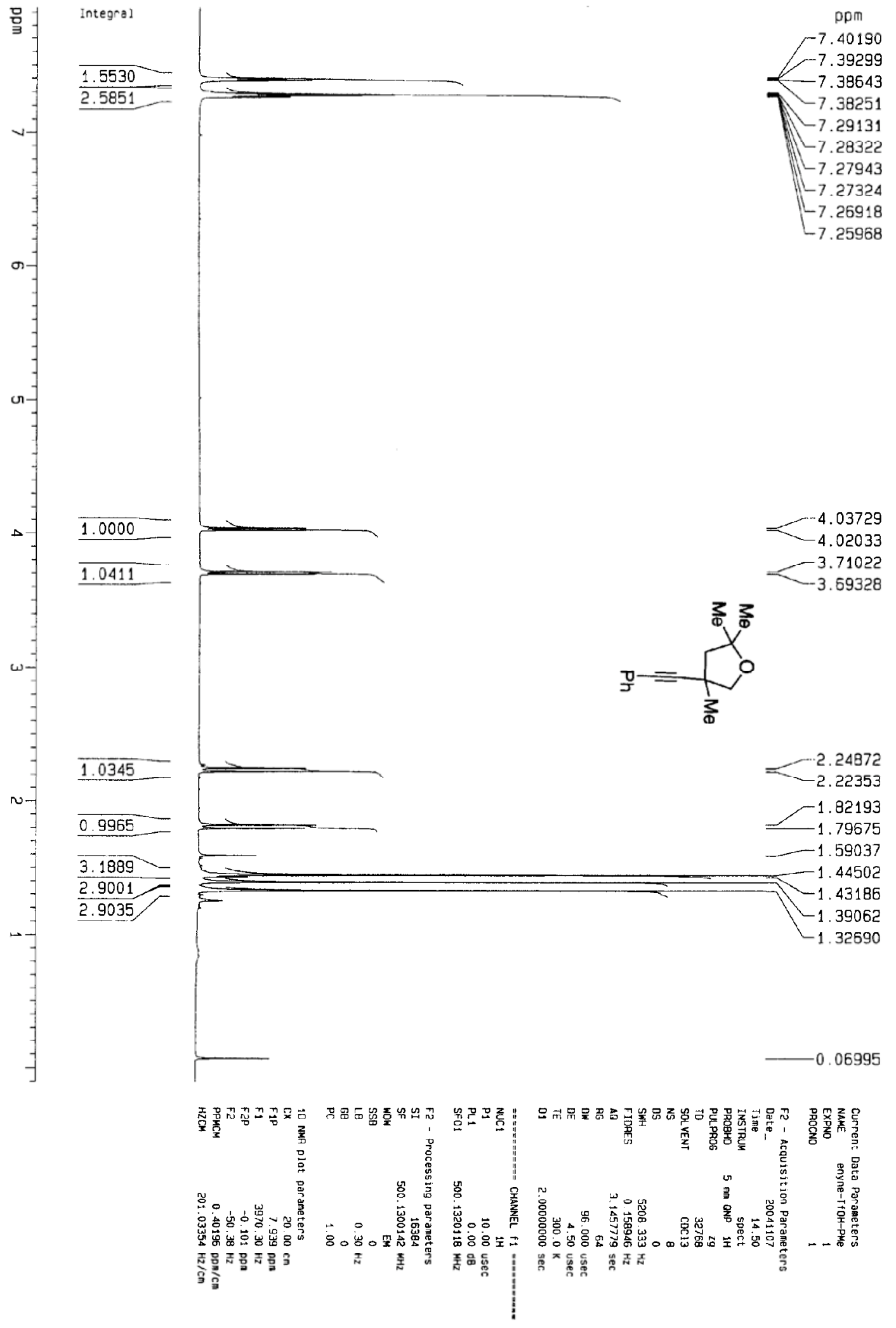




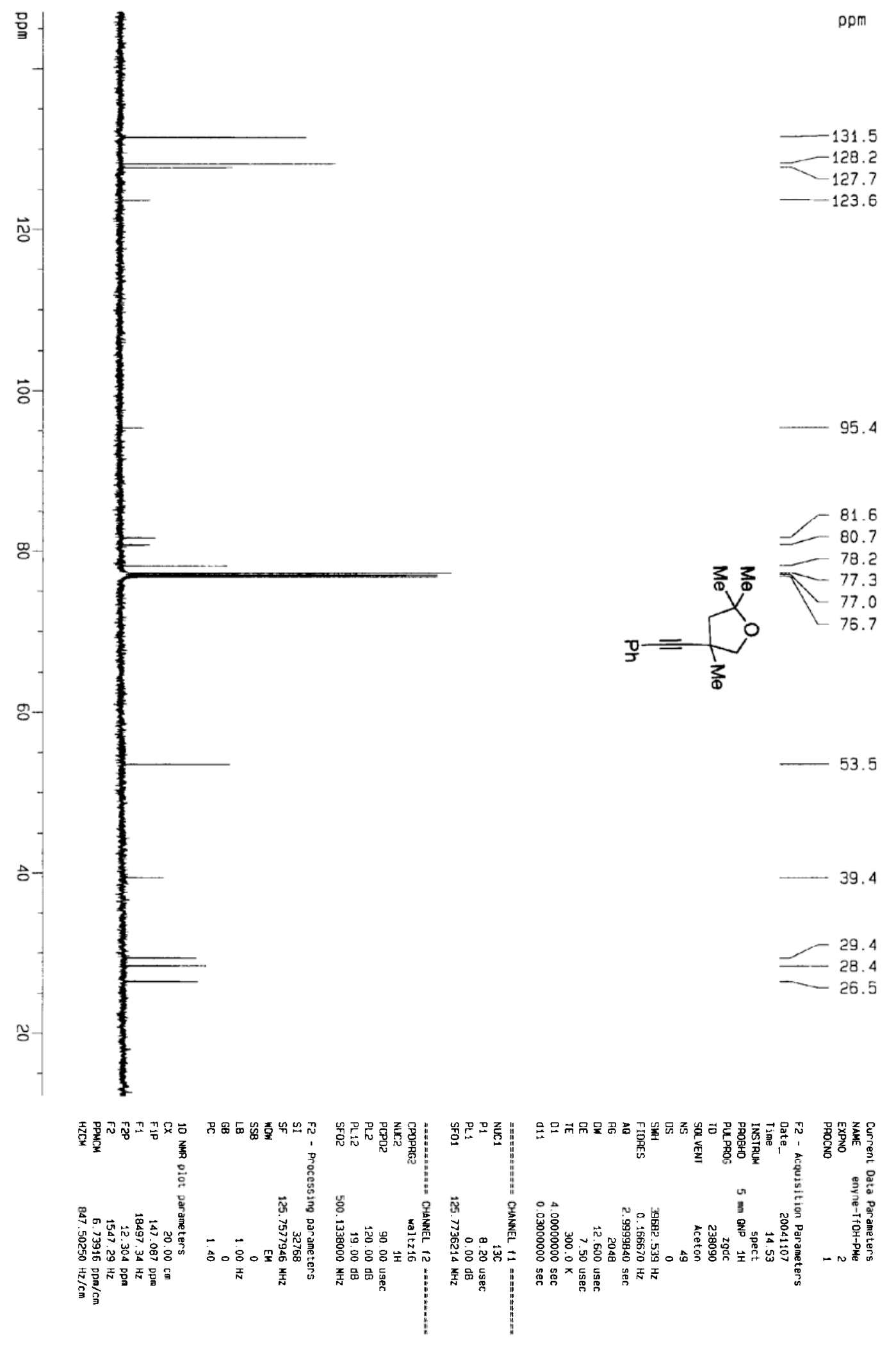




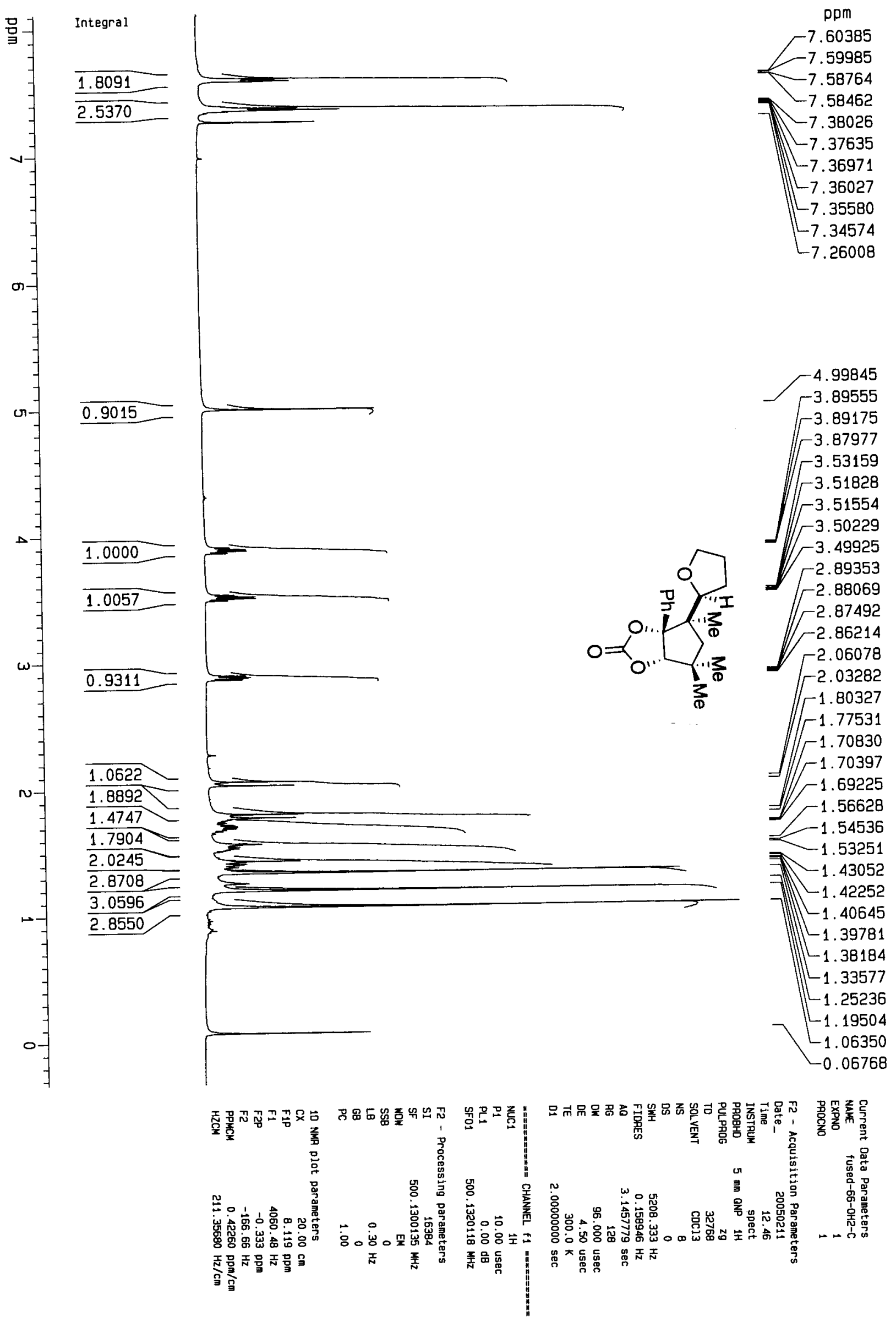




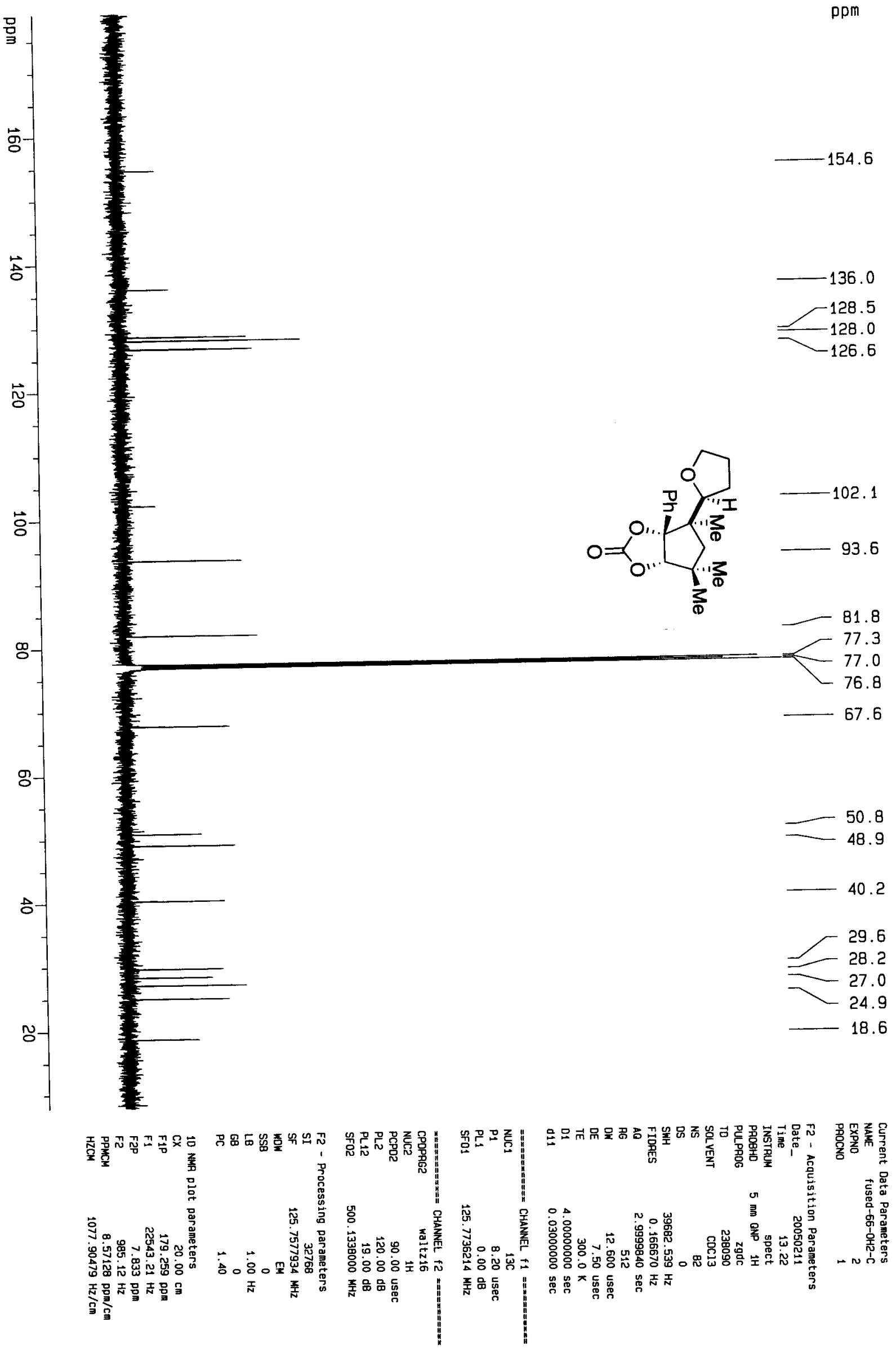




\section{Crystallographic Analysis of Carbonate 26}

\section{Data Collection}

An irregular broken fragment $(0.45 \times 0.30 \times 0.05 \mathrm{~mm})$ was selected under a stereo-microscope while immersed in Fluorolube oil to avoid possible reaction with air. The crystal was removed from the oil using a tapered glass fiber that also served to hold the crystal for data collection. The crystal was mounted and centered on a Bruker SMART APEX system at $100 \mathrm{~K}$. Rotation and still images showed the diffractions to be sharp. Frames separated in reciprocal space were obtained and provided an orientation matrix and initial cell parameters. Final cell parameters were obtained from the full data set.

A "full sphere" data set was obtained which samples approximately all of reciprocal space to a resolution of $0.84 \AA$ using $0.3^{\circ}$ steps in $\omega$ using 10 second integration times for each frame. Data collection was made at $100 \mathrm{~K}$. Integration of intensities and refinement of cell parameters were done using SAINT [1]. Absorption corrections were applied using SADABS [1] based on redundant diffractions.

\section{Structure solution and refinement}

The space group was determined as P2(1) based on systematic absences and intensity statistics. Direct methods were used to locate all non-hydrogen atoms from the E-map. Repeated difference Fourier maps allowed recognition of all expected $\mathrm{C}$ and $\mathrm{O}$ atoms. Final refinement was anisotropic for non-hydrogen atoms and isotropic for $\mathrm{H}$ atoms. No anomalous bond lengths or thermal parameters were noted. The unit cell is appropriately described as monoclinic (b), despite the small $\beta$ angle of $90.07^{\circ}$. All ORTEP diagrams have been drawn with $50 \%$ probability ellipsoids. 


\section{Equations of interest:}

$\mathrm{R}_{\text {int }}=\Sigma\left|\mathrm{F}_{\mathrm{o}}^{2}-<\mathrm{F}_{\mathrm{o}}^{2}>\right| / \Sigma\left|\mathrm{F}_{\mathrm{o}}{ }^{2}\right|$

$\mathrm{wR} 2=\left[\Sigma\left[\mathrm{W}\left(\mathrm{F}_{\mathrm{o}}{ }^{2}-\mathrm{F}_{\mathrm{c}}{ }^{2}\right)^{2}\right] / \Sigma\left[\mathrm{W}\left(\mathrm{F}_{\mathrm{o}}{ }^{2}\right)^{2}\right]\right]^{1 / 2}$

where: $\mathrm{w}=\mathrm{q} / \mathrm{\sigma}^{2}\left(\mathrm{~F}_{\mathrm{o}}{ }^{2}\right)+(\mathrm{aP})^{2}+\mathrm{bP}$;

$\mathrm{q}, \mathrm{a}, \mathrm{b}, \mathrm{P}$ as defined in [1]

$$
\mathrm{R} 1=\Sigma|| \mathrm{F}_{\mathrm{o}}|-| \mathrm{F}_{\mathrm{c}}|| / \Sigma\left|\mathrm{F}_{\mathrm{o}}\right|
$$

$$
\begin{aligned}
\mathrm{GooF} & =\mathrm{S}=\left[\Sigma\left[\mathrm{w}\left(\mathrm{F}_{\mathrm{o}}^{2-} \mathrm{F}_{\mathrm{c}}{ }^{2}\right)^{2}\right] /(\mathrm{n}-\mathrm{p})^{1 / 2}\right. \\
\mathrm{n} & =\text { number of independent reflections; } \\
\mathrm{p} & =\text { number of parameters refined. }
\end{aligned}
$$

\section{References}

[1] All software and sources of scattering factors are contained in the SHELXTL (version 5.1) program library (G. Sheldrick, Bruker Analytical X-ray Systems, Madison, WI). 
Table 1. Crystal and structure refinement for Liming1.

\section{Identification Code}

Empirical formula

Formula weight

Temperature

Wavelength

Crystal system

Space Group

Unit cell dimensions

Volume

Z

Density (calculated)

Absorption coefficient

$\mathrm{F}(000)$

Crystal size, color, habit

Theta range for data collection

Index ranges

Reflections collected

Independent reflections

Reflections with $\mathrm{I}>4 \sigma\left(\mathrm{F}_{\mathrm{o}}\right)$

Flack parameter

Absorption correction

Max. and min. transmission

Refinement method

Weighting scheme

Data / restraints / parameters

Goodness-of-fit on $\mathrm{F}^{2}$

Final $\mathrm{R}$ indices [I $>2$ sigma(I)]

$\mathrm{R}$ indices (all data)

Largest diff. peak and hole
Liming1

$\mathrm{C}_{19} \mathrm{O}_{4} \mathrm{H}_{24}$

366.38

$100 \mathrm{~K}$

$0.71073 \AA$

Monoclinic (b)

P2(1)

$$
\begin{array}{ll}
a=8.124(2) \AA & \alpha=90.00^{\circ} \\
b=12.920(3) \AA & \beta=90.069(4)^{\circ} \\
c=15.721(4) \AA & \gamma=90.00^{\circ}
\end{array}
$$$$
1650.0(7) \AA^{3}
$$

4

$1.274 \mathrm{Mg} / \mathrm{m}^{3}$

$0.088 \mathrm{~mm}^{-1}$

680

$0.45 \times 0.30 \times 0.05 \mathrm{~mm}$, transparent, plate

$1.30-28.82^{\circ}$

$-11 \leq \mathrm{h} \leq 10,-17 \leq \mathrm{k} \leq 17,-21 \leq 1 \leq 20$

19819

$7989\left(R_{\text {int }}=0.0333\right)$

6613

SADABS based on redundant diffractions

$1.0,0.833317$

Full-matrix least squares on $\mathrm{F}^{2}$

$\mathrm{w}=\mathrm{q}\left[\sigma^{2}\left(\mathrm{~F}_{\mathrm{o}}{ }^{2}\right)+(\mathrm{aP})^{2}+\mathrm{bP}\right]^{-1}$ where:

$\mathrm{P}=\left(\mathrm{F}_{\mathrm{o}}{ }^{2}+2 \mathrm{~F}_{\mathrm{c}}{ }^{2}\right) / 3, \mathrm{a}=0.0292, \mathrm{~b}=0.0, \mathrm{q}=1$

7989 / 1 / 421

1.005

$\mathrm{R} 1=0.0497, \mathrm{wR} 2=0.0801$

$\mathrm{R} 1=0.0647, \mathrm{wR} 2=0.0844$

$0.230,-0.250 \mathrm{e} \AA^{-3}$ 


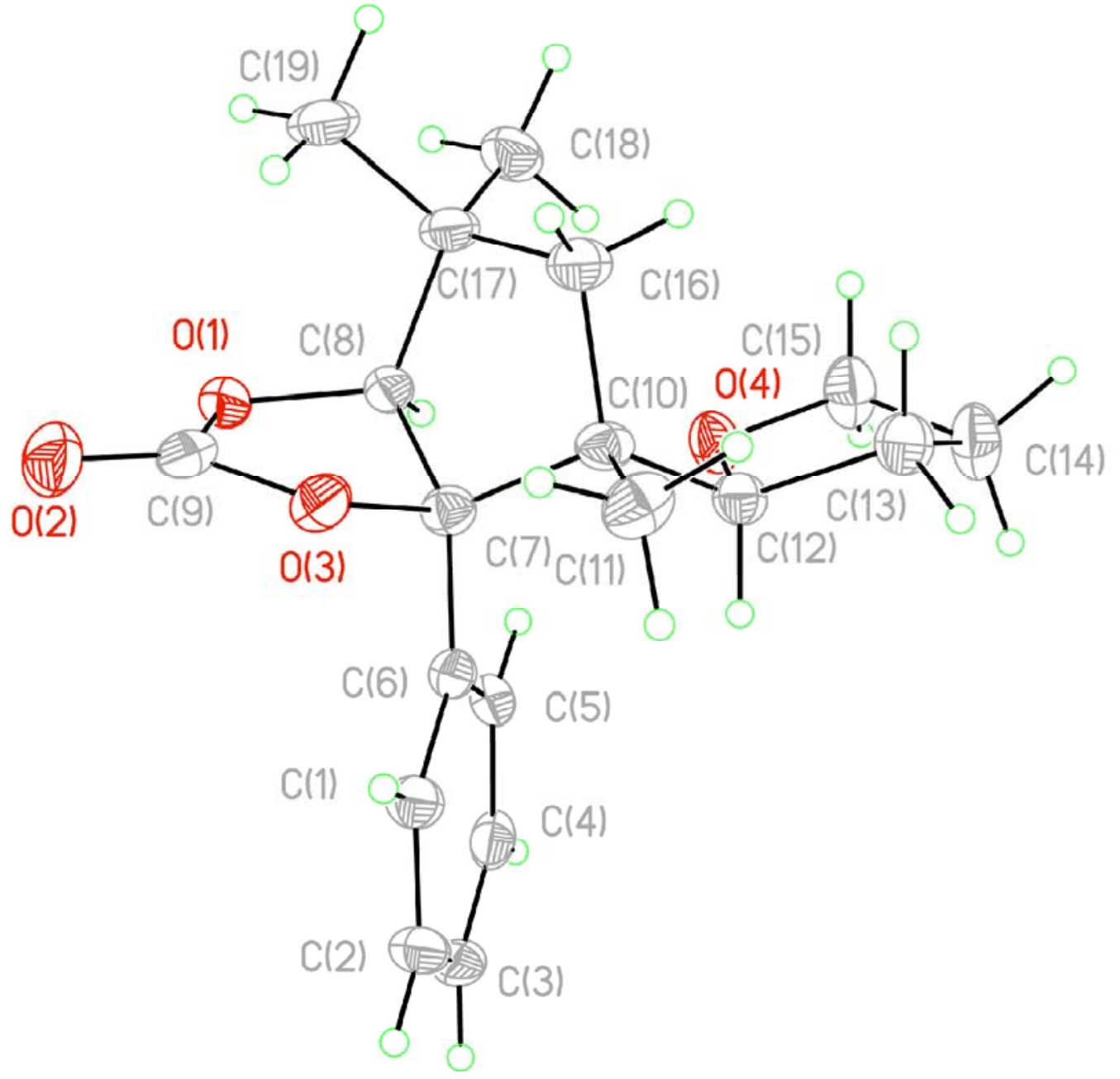




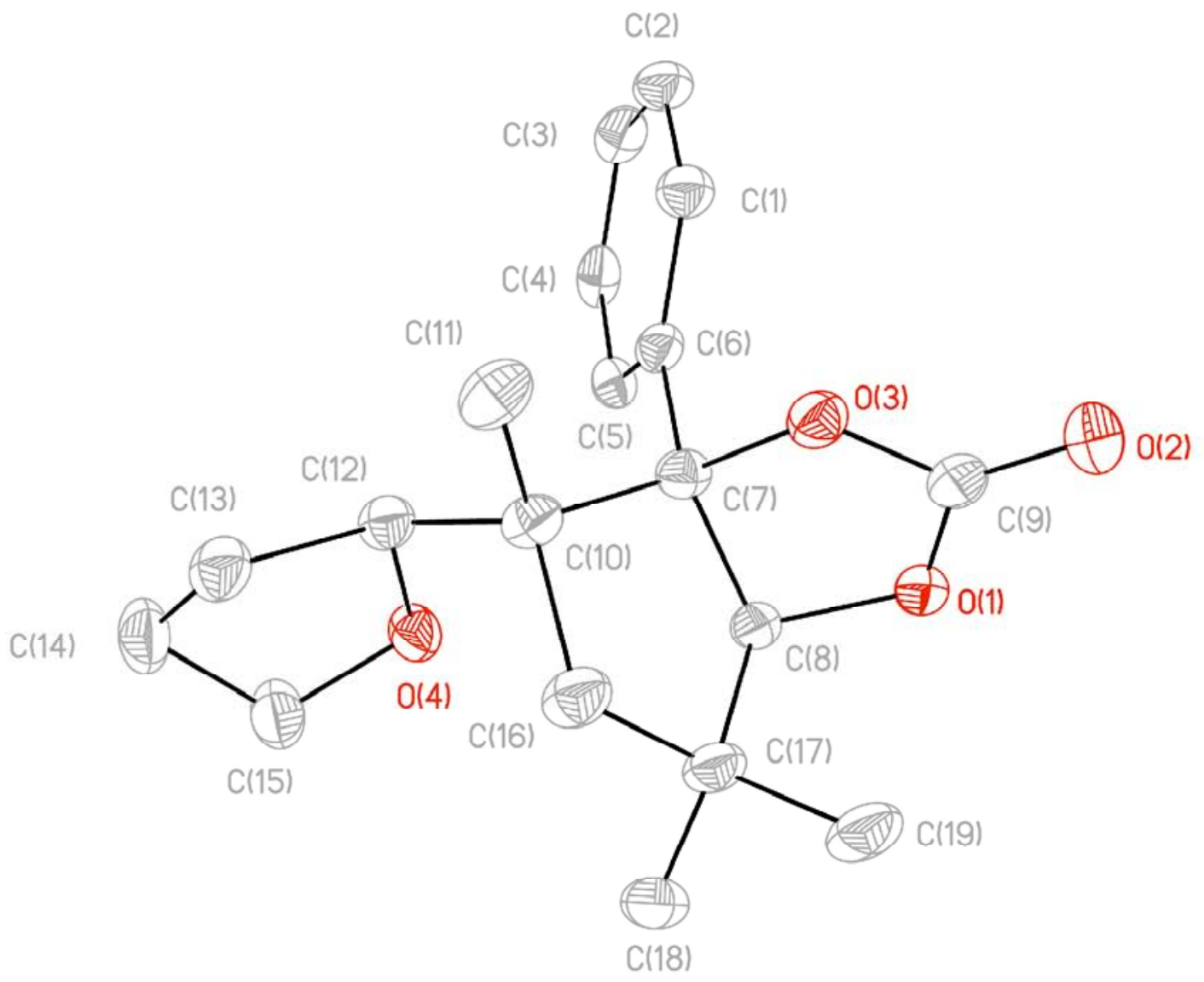




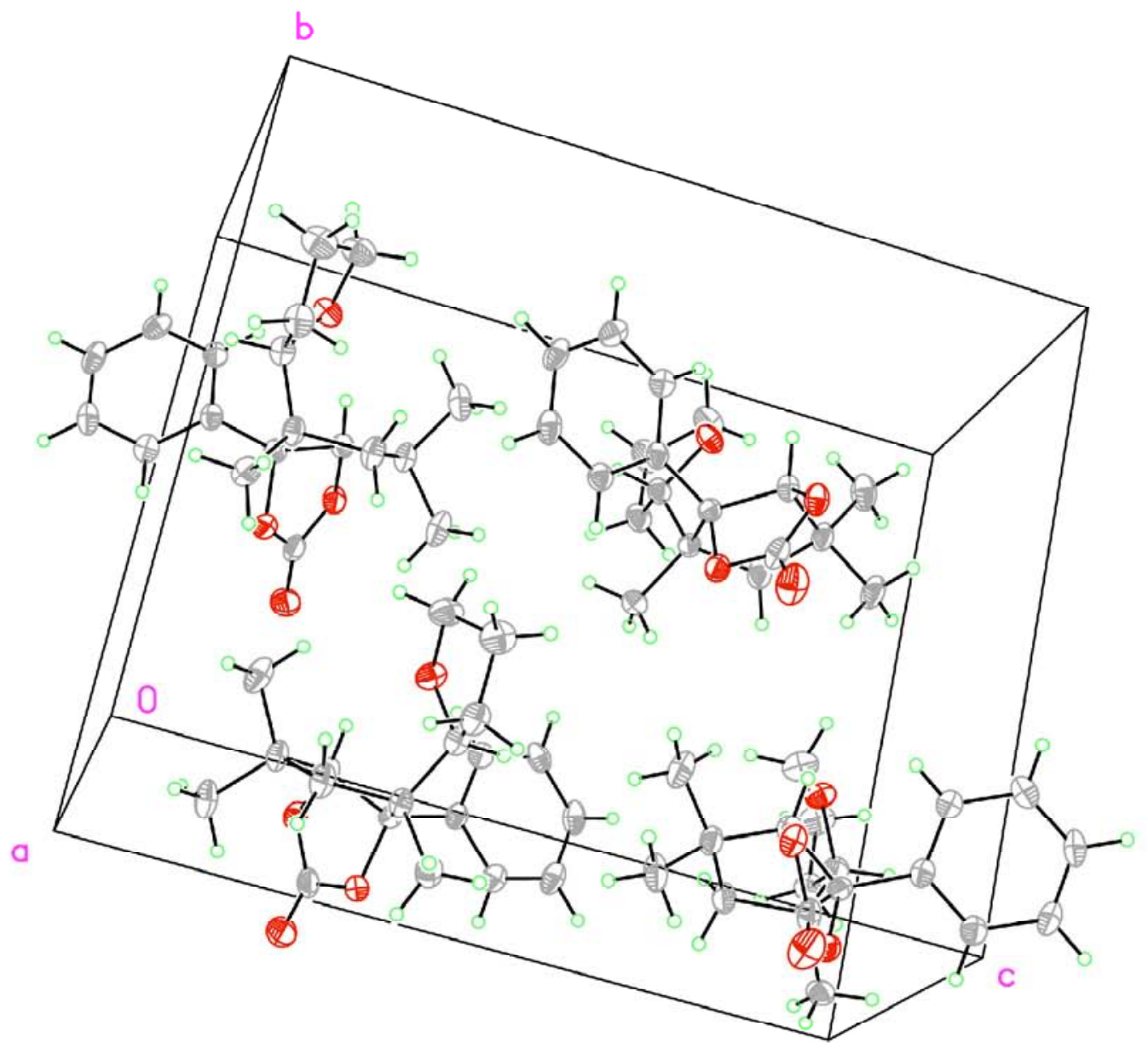


Table 2. Atomic coordinates $\left[\mathrm{x} 10^{4}\right]$ and equivalent isotropic displacement parameters $\left[\AA^{2} \times 10^{3}\right]$ for Limingl. $\mathrm{U}(\mathrm{eq})$ is defined as one third of the trace of the orthogonalized $U_{i j}$ tensor.

\begin{tabular}{|c|c|c|c|c|c|}
\hline & $\mathrm{x}$ & $\mathrm{y}$ & z & $\mathrm{U}(\mathrm{eq})$ & $\mathrm{SOF}$ \\
\hline$C(1)$ & $1954(2)$ & $5721(2)$ & $4806(1)$ & $25(1)$ & \\
\hline$C(2)$ & $1355(2)$ & $6072(2)$ & $4033(1)$ & $30(1)$ & \\
\hline $\mathrm{C}(3)$ & $1117(2)$ & $7115(2)$ & $3905(1)$ & $29(1)$ & \\
\hline$C(4)$ & $1491(2)$ & $7808(2)$ & 4541 (1) & $26(1)$ & \\
\hline$C(5)$ & $2125(2)$ & $7463(2)$ & 5311 (1) & $23(1)$ & \\
\hline$C(6)$ & $2346(2)$ & $6413(2)$ & $5457(1)$ & $21(1)$ & \\
\hline$C(7)$ & $3019(2)$ & $6002(2)$ & $6294(1)$ & $21(1)$ & \\
\hline$C(8)$ & $2440(2)$ & $6536(2)$ & $7107(1)$ & $21(1)$ & \\
\hline $\mathrm{C}(9)$ & $911(3)$ & $5095(2)$ & $6922(1)$ & $28(1)$ & \\
\hline$C(10)$ & $4921(2)$ & $5891(2)$ & $6360(1)$ & $22(1)$ & \\
\hline$C(11)$ & $5538(3)$ & $4912(2)$ & $5911(1)$ & $30(1)$ & \\
\hline$C(12)$ & $5777(2)$ & $6817(2)$ & $5949(1)$ & $23(1)$ & \\
\hline$C(13)$ & $7657(2)$ & $6812(2)$ & $5970(1)$ & $30(1)$ & \\
\hline$C(14)$ & $8058(2)$ & $7955(2)$ & $5956(2)$ & $37(1)$ & \\
\hline$C(15)$ & $6701(2)$ & $8416(2)$ & $6493(2)$ & $35(1)$ & \\
\hline$C(16)$ & $5213(2)$ & $5836(2)$ & $7329(1)$ & $30(1)$ & \\
\hline$C(17)$ & $3715(2)$ & $6304(2)$ & $7817(1)$ & $26(1)$ & \\
\hline$C(18)$ & $4141(3)$ & $7303(2)$ & $8286(1)$ & $36(1)$ & \\
\hline$C(19)$ & $3091(3)$ & $5519(2)$ & $8469(1)$ & $34(1)$ & \\
\hline$C(20)$ & $2122(2)$ & $2463(2)$ & $9687(1)$ & $23(1)$ & \\
\hline$C(21)$ & $1491(2)$ & $2806(2)$ & $10457(1)$ & $26(1)$ & \\
\hline$C(22)$ & $1117(2)$ & $2116(2)$ & $11095(1)$ & $29(1)$ & \\
\hline$C(23)$ & $1354(2)$ & $1072(2)$ & $10968(1)$ & $29(1)$ & \\
\hline$C(24)$ & $1954(2)$ & $722(2)$ & $10196(1)$ & $26(1)$ & \\
\hline$C(25)$ & $2346(2)$ & $1413(2)$ & $9544(1)$ & $22(1)$ & \\
\hline$C(26)$ & $3019(2)$ & $999(2)$ & $8707(1)$ & $21(1)$ & \\
\hline$C(27)$ & $909(3)$ & $96(2)$ & $8080(1)$ & $28(1)$ & \\
\hline$C(28)$ & $2439(2)$ & $1533(2)$ & $7893(1)$ & $22(1)$ & \\
\hline$C(29)$ & $4921(2)$ & $891(2)$ & $8641(1)$ & $22(1)$ & \\
\hline$C(30)$ & $5540(3)$ & $-89(2)$ & $9091(1)$ & $29(1)$ & \\
\hline$C(31)$ & $5776(2)$ & $1816(2)$ & $9049(1)$ & $23(1)$ & \\
\hline$C(32)$ & $7657(2)$ & $1813(2)$ & $9029(1)$ & $30(1)$ & \\
\hline$C(33)$ & $8059(3)$ & $2956(2)$ & $9043(2)$ & $37(1)$ & \\
\hline$C(34)$ & $6704(2)$ & $3417(2)$ & $8507(2)$ & $35(1)$ & \\
\hline$C(35)$ & $5217(2)$ & $835(2)$ & $7669(1)$ & $29(1)$ & \\
\hline$C(36)$ & $3716(2)$ & $1304(2)$ & $7184(1)$ & $25(1)$ & \\
\hline$C(37)$ & $4142(3)$ & $2300(2)$ & $6715(1)$ & $35(1)$ & \\
\hline$C(38)$ & $3092(3)$ & $515(2)$ & $6532(1)$ & $35(1)$ & \\
\hline$O(1)$ & $863(1)$ & $6045(1)$ & $7261(1)$ & $25(1)$ & \\
\hline$O(2)$ & $-99(2)$ & $4444(1)$ & $7030(1)$ & $41(1)$ & \\
\hline$O(3)$ & $2275(2)$ & $4974(1)$ & $6433(1)$ & $26(1)$ & \\
\hline$O(4)$ & $5296(2)$ & $7751(1)$ & $6384(1)$ & $27(1)$ & \\
\hline$O(5)$ & $2274(2)$ & $-26(1)$ & $8566(1)$ & $26(1)$ & \\
\hline$O(6)$ & $-97(2)$ & $-557(1)$ & $7970(1)$ & $42(1)$ & \\
\hline$O(7)$ & $862(1)$ & $1045(1)$ & $7738(1)$ & $26(1)$ & \\
\hline$O(8)$ & $5296(2)$ & $2751(1)$ & $8617(1)$ & $26(1)$ & \\
\hline
\end{tabular}


Table 3. Bond lengths $[\AA]$ and angles $\left[{ }^{\circ}\right]$ for Limingl.

\begin{tabular}{|c|c|}
\hline$C(1)-C(2)$ & $1.385(3)$ \\
\hline$C(1)-C(6)$ & $1.395(3)$ \\
\hline$C(2)-C(3)$ & $1.376(3)$ \\
\hline$C(3)-C(4)$ & $1.376(3)$ \\
\hline$C(4)-C(5)$ & $1.389(3)$ \\
\hline$C(5)-C(6)$ & $1.387(3)$ \\
\hline$C(6)-C(7)$ & $1.520(3)$ \\
\hline$C(7)-O(3)$ & $1.476(2)$ \\
\hline$C(7)-C(8)$ & $1.528(2)$ \\
\hline$C(7)-C(10)$ & $1.555(2)$ \\
\hline$C(8)-O(1)$ & $1.450(2)$ \\
\hline$C(8)-C(17)$ & $1.551(3)$ \\
\hline$C(9)-O(2)$ & $1.188(2)$ \\
\hline$C(9)-O(1)$ & $1.339(2)$ \\
\hline$C(9)-O(3)$ & $1.358(2)$ \\
\hline$C(10)-C(12)$ & $1.528(3)$ \\
\hline$C(10)-C(11)$ & $1.533(3)$ \\
\hline$C(10)-C(16)$ & $1.543(2)$ \\
\hline$C(12)-O(4)$ & $1.441(2)$ \\
\hline$C(12)-C(13)$ & $1.528(3)$ \\
\hline$C(13)-C(14)$ & $1.512(3)$ \\
\hline$C(14)-C(15)$ & $1.512(3)$ \\
\hline$C(15)-O(4)$ & $1.439(2)$ \\
\hline$C(16)-C(17)$ & $1.561(3)$ \\
\hline $\mathrm{C}(17)-\mathrm{C}(18)$ & $1.526(3)$ \\
\hline$C(17)-C(19)$ & $1.528(3)$ \\
\hline$C(20)-C(25)$ & $1.387(3)$ \\
\hline$C(20)-C(21)$ & $1.388(3)$ \\
\hline$C(21)-C(22)$ & $1.376(3)$ \\
\hline$C(22)-C(23)$ & $1.377(3)$ \\
\hline$C(23)-C(24)$ & $1.383(3)$ \\
\hline$C(24)-C(25)$ & $1.396(3)$ \\
\hline$C(25)-C(26)$ & $1.521(3)$ \\
\hline$C(26)-O(5)$ & $1.473(2)$ \\
\hline$C(26)-C(28)$ & $1.527(2)$ \\
\hline$C(26)-C(29)$ & $1.555(2)$ \\
\hline$C(27)-O(6)$ & $1.187(2)$ \\
\hline$C(27)-O(7)$ & $1.339(2)$ \\
\hline$C(27)-O(5)$ & $1.355(2)$ \\
\hline$C(28)-O(7)$ & $1.449(2)$ \\
\hline$C(28)-C(36)$ & $1.552(3)$ \\
\hline$C(29)-C(31)$ & $1.524(3)$ \\
\hline$C(29)-C(30)$ & $1.534(3)$ \\
\hline$C(29)-C(35)$ & $1.548(2)$ \\
\hline$C(31)-0(8)$ & $1.439(2)$ \\
\hline$C(31)-C(32)$ & $1.529(3)$ \\
\hline$C(32)-C(33)$ & $1.513(3)$ \\
\hline$C(33)-C(34)$ & $1.509(3)$ \\
\hline$C(34)-0(8)$ & $1.443(2)$ \\
\hline$C(35)-C(36)$ & $1.560(3)$ \\
\hline$C(36)-C(37)$ & $1.523(3)$ \\
\hline$C(36)-C(38)$ & $1.532(3)$ \\
\hline
\end{tabular}




\begin{tabular}{|c|c|}
\hline$C(2)-C(1)-C(6)$ & $120.9(2)$ \\
\hline$C(3)-C(2)-C(1)$ & $119.93(19)$ \\
\hline$C(4)-C(3)-C(2)$ & $119.94(18)$ \\
\hline$C(3)-C(4)-C(5)$ & $120.4(2)$ \\
\hline$C(6)-C(5)-C(4)$ & $120.33(19)$ \\
\hline$C(5)-C(6)-C(1)$ & $118.44(18)$ \\
\hline$C(5)-C(6)-C(7)$ & $122.01(17)$ \\
\hline$C(1)-C(6)-C(7)$ & $119.54(17)$ \\
\hline$O(3)-C(7)-C(6)$ & $107.19(14)$ \\
\hline$O(3)-C(7)-C(8)$ & $98.93(14)$ \\
\hline$C(6)-C(7)-C(8)$ & $117.16(16)$ \\
\hline$O(3)-C(7)-C(10)$ & $108.29(14)$ \\
\hline$C(6)-C(7)-C(10)$ & $116.55(14)$ \\
\hline$C(8)-C(7)-C(10)$ & $107.01(14)$ \\
\hline$O(1)-C(8)-C(7)$ & $102.42(14)$ \\
\hline $\mathrm{O}(1)-\mathrm{C}(8)-\mathrm{C}(17)$ & $112.68(15)$ \\
\hline$C(7)-C(8)-C(17)$ & $107.98(15)$ \\
\hline$O(2)-C(9)-O(1)$ & $124.92(19)$ \\
\hline$O(2)-C(9)-O(3)$ & $124.3(2)$ \\
\hline $\mathrm{O}(1)-\mathrm{C}(9)-\mathrm{O}(3)$ & $110.78(17)$ \\
\hline$C(12)-C(10)-C(11)$ & $107.58(15)$ \\
\hline$C(12)-C(10)-C(16)$ & $112.58(16)$ \\
\hline$C(11)-C(10)-C(16)$ & $111.49(16)$ \\
\hline$C(12)-C(10)-C(7)$ & $110.60(15)$ \\
\hline$C(11)-C(10)-C(7)$ & $111.79(15)$ \\
\hline$C(16)-C(10)-C(7)$ & $102.85(13)$ \\
\hline$O(4)-C(12)-C(13)$ & $105.37(15)$ \\
\hline$O(4)-C(12)-C(10)$ & $109.33(14)$ \\
\hline$C(13)-C(12)-C(10)$ & $116.32(16)$ \\
\hline$C(14)-C(13)-C(12)$ & $102.19(16)$ \\
\hline$C(15)-C(14)-C(13)$ & $102.69(17)$ \\
\hline$O(4)-C(15)-C(14)$ & $106.10(17)$ \\
\hline$C(10)-C(16)-C(17)$ & $110.36(15)$ \\
\hline$C(18)-C(17)-C(19)$ & $108.24(17)$ \\
\hline$C(18)-C(17)-C(8)$ & $109.54(16)$ \\
\hline$C(19)-C(17)-C(8)$ & $112.85(16)$ \\
\hline$C(18)-C(17)-C(16)$ & $112.85(17)$ \\
\hline$C(19)-C(17)-C(16)$ & $109.39(17)$ \\
\hline$C(8)-C(17)-C(16)$ & $104.01(15)$ \\
\hline$C(25)-C(20)-C(21)$ & $120.21(19)$ \\
\hline$C(22)-C(21)-C(20)$ & $120.7(2)$ \\
\hline$C(21)-C(22)-C(23)$ & $119.84(18)$ \\
\hline$C(22)-C(23)-C(24)$ & $119.78(19)$ \\
\hline$C(23)-C(24)-C(25)$ & $121.1(2)$ \\
\hline$C(20)-C(25)-C(24)$ & $118.34(18)$ \\
\hline$C(20)-C(25)-C(26)$ & $122.08(17)$ \\
\hline$C(24)-C(25)-C(26)$ & $119.57(18)$ \\
\hline$O(5)-C(26)-C(25)$ & $107.34(14)$ \\
\hline $\mathrm{O}(5)-\mathrm{C}(26)-\mathrm{C}(28)$ & $98.82(14)$ \\
\hline$C(25)-C(26)-C(28)$ & $117.06(16)$ \\
\hline$O(5)-C(26)-C(29)$ & $108.45(14)$ \\
\hline$C(25)-C(26)-C(29)$ & $116.61(14)$ \\
\hline$C(28)-C(26)-C(29)$ & $106.85(14)$ \\
\hline$O(6)-C(27)-O(7)$ & $124.97(19)$ \\
\hline$O(6)-C(27)-O(5)$ & $124.2(2)$ \\
\hline $\mathrm{O}(7)-\mathrm{C}(27)-\mathrm{O}(5)$ & $110.82(17)$ \\
\hline$O(7)-C(28)-C(26)$ & $102.49(14)$ \\
\hline
\end{tabular}




$\begin{array}{ll}O(7)-C(28)-C(36) & 112.79(15) \\ C(26)-C(28)-C(36) & 108.07(15) \\ C(31)-C(29)-C(30) & 107.66(15) \\ C(31)-C(29)-C(35) & 112.35(16) \\ C(30)-C(29)-C(35) & 111.45(16) \\ C(31)-C(29)-C(26) & 110.68(14) \\ C(30)-C(29)-C(26) & 111.65(15) \\ C(35)-C(29)-C(26) & 103.08(13) \\ O(8)-C(31)-C(29) & 109.68(14) \\ O(8)-C(31)-C(32) & 105.22(15) \\ C(29)-C(31)-C(32) & 116.42(16) \\ C(33)-C(32)-C(31) & 102.33(16) \\ C(34)-C(33)-C(32) & 102.67(17) \\ O(8)-C(34)-C(33) & 105.99(17) \\ C(29)-C(35)-C(36) & 110.00(15) \\ C(37)-C(36)-C(38) & 108.23(17) \\ C(37)-C(36)-C(28) & 109.85(16) \\ C(38)-C(36)-C(28) & 112.79(16) \\ C(37)-C(36)-C(35) & 112.75(17) \\ C(38)-C(36)-C(35) & 109.06(16) \\ C(28)-C(36)-C(35) & 104.21(15) \\ C(9)-O(1)-C(8) & 107.97(15) \\ C(9)-O(3)-C(7) & 108.38(14) \\ C(15)-O(4)-C(12) & 109.94(14) \\ C(27)-O(5)-C(26) & 108.46(14) \\ C(27)-O(7)-C(28) & 107.84(15) \\ C(31)-O(8)-C(34) & 110.07(14) \\ \end{array}$


Table 4. Anisotropic displacement parameters $\left[\AA^{2} \times 10^{3}\right]$ for Liming1. The anisotropic displacement factor exponent takes the form:

$-2 \pi^{2}\left[h^{2} a^{* 2} U_{11}+\ldots+2 h k a{ }^{*} b^{*} U_{12}\right]$

\begin{tabular}{|c|c|c|c|c|c|c|}
\hline & $\mathrm{U}_{11}$ & $\mathrm{U}_{22}$ & $\mathrm{U}_{33}$ & $\mathrm{U}_{23}$ & $\mathrm{U}_{13}$ & $\mathrm{U}_{12}$ \\
\hline$C(1)$ & $24(1)$ & $29(1)$ & $22(1)$ & $-2(1)$ & $-1(1)$ & $1(1)$ \\
\hline$C(2)$ & $26(1)$ & $44(1)$ & $20(1)$ & $-6(1)$ & $-3(1)$ & $-1(1)$ \\
\hline$C(3)$ & $20(1)$ & $48(1)$ & $19(1)$ & $8(1)$ & $-3(1)$ & $3(1)$ \\
\hline$C(4)$ & $18(1)$ & $32(1)$ & $29(1)$ & $9(1)$ & $3(1)$ & $1(1)$ \\
\hline C (5) & $17(1)$ & $29(1)$ & $25(1)$ & $-1(1)$ & $0(1)$ & $-2(1)$ \\
\hline$C(6)$ & $17(1)$ & $29(1)$ & $18(1)$ & $-2(1)$ & $2(1)$ & $1(1)$ \\
\hline$C(7)$ & $25(1)$ & $19(1)$ & $19(1)$ & $0(1)$ & $-1(1)$ & $-1(1)$ \\
\hline$C(8)$ & $19(1)$ & $27(1)$ & $18(1)$ & $-2(1)$ & $0(1)$ & $0(1)$ \\
\hline$C(9)$ & $34(1)$ & $34(1)$ & $17(1)$ & $4(1)$ & $-1(1)$ & $-6(1)$ \\
\hline$C(10)$ & $22(1)$ & $27(1)$ & $18(1)$ & $1(1)$ & $-2(1)$ & $7(1)$ \\
\hline$C(11)$ & $34(1)$ & $29(1)$ & $27(1)$ & $1(1)$ & $5(1)$ & $9(1)$ \\
\hline$C(12)$ & $20(1)$ & $28(1)$ & $22(1)$ & $-2(1)$ & $0(1)$ & $5(1)$ \\
\hline$C(13)$ & $24(1)$ & $38(1)$ & $29(1)$ & $-3(1)$ & $2(1)$ & $7(1)$ \\
\hline C (14) & $22(1)$ & $42(2)$ & $48(1)$ & $-7(1)$ & $8(1)$ & $-3(1)$ \\
\hline$C(15)$ & $27(1)$ & $33(1)$ & $44(1)$ & $-9(1)$ & $7(1)$ & $-6(1)$ \\
\hline$C(16)$ & $27(1)$ & $40(1)$ & $22(1)$ & $0(1)$ & $-1(1)$ & $8(1)$ \\
\hline C (17) & $27(1)$ & $35(1)$ & $16(1)$ & $1(1)$ & $-2(1)$ & $3(1)$ \\
\hline$C(18)$ & $29(1)$ & $54(2)$ & $24(1)$ & $-10(1)$ & $-3(1)$ & $-1(1)$ \\
\hline$C(19)$ & $34(1)$ & $52(2)$ & $17(1)$ & $5(1)$ & $-2(1)$ & $7(1)$ \\
\hline$C(20)$ & $18(1)$ & $27(1)$ & $24(1)$ & $0(1)$ & $-1(1)$ & $-3(1)$ \\
\hline$C(21)$ & $18(1)$ & $32(1)$ & $28(1)$ & $-10(1)$ & $-4(1)$ & $1(1)$ \\
\hline$C(22)$ & $20(1)$ & $49(2)$ & $19(1)$ & $-6(1)$ & $2(1)$ & $2(1)$ \\
\hline$C(23)$ & $24(1)$ & $44(1)$ & $19(1)$ & $6(1)$ & $2(1)$ & $0(1)$ \\
\hline$C(24)$ & $24(1)$ & $31(1)$ & $24(1)$ & $0(1)$ & $0(1)$ & $3(1)$ \\
\hline$C(25)$ & $17(1)$ & $30(1)$ & $19(1)$ & $2(1)$ & $-2(1)$ & $0(1)$ \\
\hline$C(26)$ & $24(1)$ & $19(1)$ & $20(1)$ & $2(1)$ & $-1(1)$ & $1(1)$ \\
\hline$C(27)$ & $32(1)$ & $34(1)$ & $17(1)$ & $-4(1)$ & $1(1)$ & $-4(1)$ \\
\hline$C(28)$ & $20(1)$ & $25(1)$ & $20(1)$ & $2(1)$ & $-2(1)$ & $0(1)$ \\
\hline C (29) & $21(1)$ & $28(1)$ & $18(1)$ & $0(1)$ & $1(1)$ & $7(1)$ \\
\hline$C(30)$ & $35(1)$ & $27(1)$ & 27 (1) & $-1(1)$ & $-7(1)$ & $9(1)$ \\
\hline$C(31)$ & $20(1)$ & $26(1)$ & $24(1)$ & $2(1)$ & $0(1)$ & $7(1)$ \\
\hline C (32) & $24(1)$ & $36(1)$ & $31(1)$ & $3(1)$ & $-3(1)$ & $6(1)$ \\
\hline$C(33)$ & $23(1)$ & $39(2)$ & $49(1)$ & $7(1)$ & $-8(1)$ & $-3(1)$ \\
\hline C (34) & $28(1)$ & $34(1)$ & $44(1)$ & $6(1)$ & $-10(1)$ & $-6(1)$ \\
\hline$C(35)$ & $28(1)$ & $39(1)$ & $20(1)$ & $-1(1)$ & $2(1)$ & $8(1)$ \\
\hline$C(36)$ & $27(1)$ & $32(1)$ & $17(1)$ & $0(1)$ & $0(1)$ & $3(1)$ \\
\hline C (37) & $28(1)$ & $53(2)$ & $24(1)$ & $10(1)$ & $2(1)$ & $-2(1)$ \\
\hline$C(38)$ & $34(1)$ & $52(2)$ & $20(1)$ & $-6(1)$ & $2(1)$ & $6(1)$ \\
\hline$O(1)$ & $22(1)$ & $34(1)$ & $20(1)$ & $-2(1)$ & $0(1)$ & $-4(1)$ \\
\hline$O(2)$ & $44(1)$ & $43(1)$ & $36(1)$ & $-2(1)$ & $6(1)$ & $-20(1)$ \\
\hline$O(3)$ & $32(1)$ & $24(1)$ & $21(1)$ & $0(1)$ & $3(1)$ & $-3(1)$ \\
\hline$O(4)$ & $20(1)$ & $28(1)$ & $32(1)$ & $-8(1)$ & $2(1)$ & $0(1)$ \\
\hline$O(5)$ & $32(1)$ & $24(1)$ & $21(1)$ & $-1(1)$ & $-3(1)$ & $-2(1)$ \\
\hline$O(6)$ & $45(1)$ & $45(1)$ & $35(1)$ & $2(1)$ & $-7(1)$ & $-21(1)$ \\
\hline$O(7)$ & $22(1)$ & $34(1)$ & $21(1)$ & $2(1)$ & $-1(1)$ & $-4(1)$ \\
\hline$O(8)$ & $19(1)$ & $27(1)$ & $33(1)$ & $8(1)$ & $-2(1)$ & $1(1)$ \\
\hline
\end{tabular}




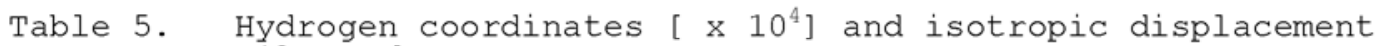
parameters $\left[\begin{array}{lll}\AA^{2} & \times & 10^{3}\end{array}\right]$ for Limingl.

\begin{tabular}{|c|c|c|c|c|}
\hline & $\mathrm{x}$ & $\mathrm{y}$ & $z$ & $\mathrm{U}(\mathrm{eq})$ \\
\hline $\mathrm{H}(1)$ & 2101 & 5000 & 4894 & 30 \\
\hline $\mathrm{H}(2)$ & 1108 & 5594 & 3592 & 36 \\
\hline $\mathrm{H}(3)$ & 695 & 7356 & 3377 & 35 \\
\hline $\mathrm{H}(4)$ & 1314 & 8527 & 4453 & 31 \\
\hline $\mathrm{H}(5)$ & 2410 & 7948 & 5741 & 28 \\
\hline $\mathrm{H}(8)$ & 2310 & 7298 & 7019 & 26 \\
\hline $\mathrm{H}(11 \mathrm{~A})$ & 4918 & 4311 & 6116 & 45 \\
\hline $\mathrm{H}(11 \mathrm{~B})$ & 6711 & 4814 & 6035 & 45 \\
\hline $\mathrm{H}(11 \mathrm{C})$ & 5382 & 4984 & 5296 & 45 \\
\hline $\mathrm{H}(12)$ & 5409 & 6867 & 5343 & 28 \\
\hline $\mathrm{H}(13 \mathrm{~A})$ & 8078 & 6478 & 6493 & 37 \\
\hline $\mathrm{H}(13 \mathrm{~B})$ & 8118 & 6454 & 5467 & 37 \\
\hline $\mathrm{H}(14 \mathrm{~A})$ & 9154 & 8092 & 6208 & 45 \\
\hline $\mathrm{H}(14 \mathrm{~B})$ & 8031 & 8233 & 5369 & 45 \\
\hline $\mathrm{H}(15 \mathrm{~A})$ & 7036 & 8442 & 7098 & 42 \\
\hline $\mathrm{H}(15 \mathrm{~B})$ & 6442 & 9128 & 6301 & 42 \\
\hline $\mathrm{H}(16 \mathrm{~A})$ & 6224 & 6225 & 7477 & 35 \\
\hline $\mathrm{H}(16 \mathrm{~B})$ & 5372 & 5106 & 7502 & 35 \\
\hline $\mathrm{H}(18 \mathrm{~A})$ & 3159 & 7566 & 8575 & 54 \\
\hline $\mathrm{H}(18 \mathrm{~B})$ & 4533 & 7820 & 7877 & 54 \\
\hline $\mathrm{H}(18 \mathrm{C})$ & 5005 & 7165 & 8706 & 54 \\
\hline $\mathrm{H}(19 \mathrm{~A})$ & 3951 & 5389 & 8893 & 52 \\
\hline $\mathrm{H}(19 \mathrm{~B})$ & 2812 & 4870 & 8180 & 52 \\
\hline $\mathrm{H}(19 \mathrm{C})$ & 2110 & 5795 & 8751 & 52 \\
\hline $\mathrm{H}(20)$ & 2401 & 2948 & 9257 & 27 \\
\hline $\mathrm{H}(21)$ & 1315 & 3525 & 10545 & 31 \\
\hline $\mathrm{H}(22)$ & 696 & 2359 & 11622 & 35 \\
\hline $\mathrm{H}(23)$ & 1107 & 593 & 11408 & 35 \\
\hline $\mathrm{H}(24)$ & 2101 & 1 & 10109 & 31 \\
\hline $\mathrm{H}(28)$ & 2310 & 2295 & 7983 & 26 \\
\hline $\mathrm{H}(30 \mathrm{~A})$ & 6715 & -182 & 8973 & 44 \\
\hline $\mathrm{H}(30 \mathrm{~B})$ & 4927 & -690 & 8882 & 44 \\
\hline $\mathrm{H}(30 \mathrm{C})$ & 5374 & -18 & 9706 & 44 \\
\hline $\mathrm{H}(31)$ & 5412 & 1865 & 9655 & 28 \\
\hline $\mathrm{H}(32 \mathrm{~A})$ & 8120 & 1455 & 9532 & 36 \\
\hline $\mathrm{H}(32 \mathrm{~B})$ & 8077 & 1479 & 8505 & 36 \\
\hline $\mathrm{H}(33 \mathrm{~A})$ & 8033 & 3233 & 9630 & 45 \\
\hline $\mathrm{H}(33 \mathrm{~B})$ & 9155 & 3093 & 8792 & 45 \\
\hline $\mathrm{H}(34 \mathrm{~A})$ & 6447 & 4129 & 8697 & 42 \\
\hline $\mathrm{H}(34 \mathrm{~B})$ & 7035 & 3440 & 7901 & 42 \\
\hline $\mathrm{H}(35 \mathrm{~A})$ & 5375 & 105 & 7496 & 35 \\
\hline $\mathrm{H}(35 \mathrm{~B})$ & 6227 & 1224 & 7523 & 35 \\
\hline $\mathrm{H}(37 \mathrm{~A})$ & 4997 & 2158 & 6291 & 53 \\
\hline $\mathrm{H}(37 \mathrm{~B})$ & 4547 & 2815 & 7122 & 53 \\
\hline $\mathrm{H}(37 \mathrm{C})$ & 3157 & 2567 & 6429 & 53 \\
\hline $\mathrm{H}(38 \mathrm{~A})$ & 2109 & 788 & 6249 & 53 \\
\hline
\end{tabular}


$\mathrm{H}$ ( $38 \mathrm{~B})$

$\mathrm{H}(38 \mathrm{C})$

2818

3952

$-134$

386

6822

6108

53

53 
Table 6. Torsion angles $\left[{ }^{\circ}\right]$ for Limingl.

\begin{tabular}{|c|c|}
\hline$C(6)-C(1)-C(2)-C(3)$ & $-0.9(3)$ \\
\hline $\mathrm{C}(1)-\mathrm{C}(2)-\mathrm{C}(3)-\mathrm{C}(4)$ & $0.7(3)$ \\
\hline$C(2)-C(3)-C(4)-C(5)$ & $0.7(3)$ \\
\hline$C(3)-C(4)-C(5)-C(6)$ & $-1.8(3)$ \\
\hline$C(4)-C(5)-C(6)-C(1)$ & $1.5(3)$ \\
\hline$C(4)-C(5)-C(6)-C(7)$ & $-179.35(16)$ \\
\hline$C(2)-C(1)-C(6)-C(5)$ & $-0.2(3)$ \\
\hline $\mathrm{C}(2)-\mathrm{C}(1)-\mathrm{C}(6)-\mathrm{C}(7)$ & $-179.31(17)$ \\
\hline$C(5)-C(6)-C(7)-O(3)$ & $149.10(16)$ \\
\hline $\mathrm{C}(1)-\mathrm{C}(6)-\mathrm{C}(7)-\mathrm{O}(3)$ & $-31.8(2)$ \\
\hline $\mathrm{C}(5)-\mathrm{C}(6)-\mathrm{C}(7)-\mathrm{C}(8)$ & $39.1(2)$ \\
\hline$C(1)-C(6)-C(7)-C(8)$ & $-141.75(17)$ \\
\hline$C(5)-C(6)-C(7)-C(10)$ & $-89.5(2)$ \\
\hline$C(1)-C(6)-C(7)-C(10)$ & $89.7(2)$ \\
\hline$O(3)-C(7)-C(8)-O(1)$ & $-32.49(16)$ \\
\hline $\mathrm{C}(6)-\mathrm{C}(7)-\mathrm{C}(8)-\mathrm{O}(1)$ & $82.14(19)$ \\
\hline $\mathrm{C}(10)-\mathrm{C}(7)-\mathrm{C}(8)-\mathrm{O}(1)$ & $-144.84(15)$ \\
\hline $\mathrm{O}(3)-\mathrm{C}(7)-\mathrm{C}(8)-\mathrm{C}(17)$ & $86.62(16)$ \\
\hline $\mathrm{C}(6)-\mathrm{C}(7)-\mathrm{C}(8)-\mathrm{C}(17)$ & $-158.75(16)$ \\
\hline$C(10)-C(7)-C(8)-C(17)$ & $-25.7(2)$ \\
\hline $\mathrm{O}(3)-\mathrm{C}(7)-\mathrm{C}(10)-\mathrm{C}(12)$ & $161.75(14)$ \\
\hline$C(6)-C(7)-C(10)-C(12)$ & $40.9(2)$ \\
\hline$C(8)-C(7)-C(10)-C(12)$ & $-92.47(17)$ \\
\hline$O(3)-C(7)-C(10)-C(11)$ & $41.90(19)$ \\
\hline$C(6)-C(7)-C(10)-C(11)$ & $-79.0(2)$ \\
\hline$C(8)-C(7)-C(10)-C(11)$ & $147.68(16)$ \\
\hline$O(3)-C(7)-C(10)-C(16)$ & $-77.83(17)$ \\
\hline$C(6)-C(7)-C(10)-C(16)$ & $161.30(17)$ \\
\hline$C(8)-C(7)-C(10)-C(16)$ & $28.0(2)$ \\
\hline$C(11)-C(10)-C(12)-O(4)$ & $-176.93(15)$ \\
\hline $\mathrm{C}(16)-\mathrm{C}(10)-\mathrm{C}(12)-\mathrm{O}(4)$ & $-53.70(19)$ \\
\hline $\mathrm{C}(7)-\mathrm{C}(10)-\mathrm{C}(12)-\mathrm{O}(4)$ & $60.73(18)$ \\
\hline$C(11)-C(10)-C(12)-C(13)$ & $-57.8(2)$ \\
\hline $\mathrm{C}(16)-\mathrm{C}(10)-\mathrm{C}(12)-\mathrm{C}(13)$ & $65.4(2)$ \\
\hline $\mathrm{C}(7)-\mathrm{C}(10)-\mathrm{C}(12)-\mathrm{C}(13)$ & $179.86(15)$ \\
\hline $\mathrm{O}(4)-\mathrm{C}(12)-\mathrm{C}(13)-\mathrm{C}(14)$ & $-30.99(19)$ \\
\hline $\mathrm{C}(10)-\mathrm{C}(12)-\mathrm{C}(13)-\mathrm{C}(14)$ & $-152.25(17)$ \\
\hline $\mathrm{C}(12)-\mathrm{C}(13)-\mathrm{C}(14)-\mathrm{C}(15)$ & $36.6(2)$ \\
\hline $\mathrm{C}(13)-\mathrm{C}(14)-\mathrm{C}(15)-\mathrm{O}(4)$ & $-29.8(2)$ \\
\hline$C(12)-C(10)-C(16)-C(17)$ & $98.40(19)$ \\
\hline$C(11)-C(10)-C(16)-C(17)$ & $-140.59(17)$ \\
\hline $\mathrm{C}(7)-\mathrm{C}(10)-\mathrm{C}(16)-\mathrm{C}(17)$ & $-20.7(2)$ \\
\hline $\mathrm{O}(1)-\mathrm{C}(8)-\mathrm{C}(17)-\mathrm{C}(18)$ & $-114.50(18)$ \\
\hline $\mathrm{C}(7)-\mathrm{C}(8)-\mathrm{C}(17)-\mathrm{C}(18)$ & $133.12(17)$ \\
\hline $\mathrm{O}(1)-\mathrm{C}(8)-\mathrm{C}(17)-\mathrm{C}(19)$ & $6.2(2)$ \\
\hline $\mathrm{C}(7)-\mathrm{C}(8)-\mathrm{C}(17)-\mathrm{C}(19)$ & $-106.22(18)$ \\
\hline$O(1)-C(8)-C(17)-C(16)$ & $124.62(17)$ \\
\hline$C(7)-C(8)-C(17)-C(16)$ & $12.2(2)$ \\
\hline$C(10)-C(16)-C(17)-C(18)$ & $-112.96(19)$ \\
\hline$C(10)-C(16)-C(17)-C(19)$ & $126.48(18)$ \\
\hline $\mathrm{C}(10)-\mathrm{C}(16)-\mathrm{C}(17)-\mathrm{C}(8)$ & $5.7(2)$ \\
\hline$C(25)-C(20)-C(21)-C(22)$ & $1.6(3)$ \\
\hline $\mathrm{C}(20)-\mathrm{C}(21)-\mathrm{C}(22)-\mathrm{C}(23)$ & $-0.7(3)$ \\
\hline
\end{tabular}




$$
\begin{aligned}
& \mathrm{C}(21)-\mathrm{C}(22)-\mathrm{C}(23)-\mathrm{C}(24) \\
& C(22)-C(23)-C(24)-C(25) \\
& C(21)-C(20)-C(25)-C(24) \\
& C(21)-C(20)-C(25)-C(26) \\
& \mathrm{C}(23)-\mathrm{C}(24)-\mathrm{C}(25)-\mathrm{C}(20) \\
& C(23)-C(24)-C(25)-C(26) \\
& C(20)-C(25)-C(26)-O(5) \\
& \mathrm{C}(24)-\mathrm{C}(25)-\mathrm{C}(26)-\mathrm{O}(5) \\
& \mathrm{C}(20)-\mathrm{C}(25)-\mathrm{C}(26)-\mathrm{C}(28) \\
& \mathrm{C}(24)-\mathrm{C}(25)-\mathrm{C}(26)-\mathrm{C}(28) \\
& C(20)-C(25)-C(26)-C(29) \\
& C(24)-C(25)-C(26)-C(29) \\
& \mathrm{O}(5)-\mathrm{C}(26)-\mathrm{C}(28)-\mathrm{O}(7) \\
& \mathrm{C}(25)-\mathrm{C}(26)-\mathrm{C}(28)-\mathrm{O}(7) \\
& \mathrm{C}(29)-\mathrm{C}(26)-\mathrm{C}(28)-\mathrm{O}(7) \\
& \mathrm{O}(5)-\mathrm{C}(26)-\mathrm{C}(28)-\mathrm{C}(36) \\
& \mathrm{C}(25)-\mathrm{C}(26)-\mathrm{C}(28)-\mathrm{C}(36) \\
& C(29)-C(26)-C(28)-C(36) \\
& \mathrm{O}(5)-\mathrm{C}(26)-\mathrm{C}(29)-\mathrm{C}(31) \\
& C(25)-C(26)-C(29)-C(31) \\
& \mathrm{C}(28)-\mathrm{C}(26)-\mathrm{C}(29)-\mathrm{C}(31) \\
& \mathrm{O}(5)-\mathrm{C}(26)-\mathrm{C}(29)-\mathrm{C}(30) \\
& C(25)-C(26)-C(29)-C(30) \\
& C(28)-C(26)-C(29)-C(30) \\
& \mathrm{O}(5)-\mathrm{C}(26)-\mathrm{C}(29)-\mathrm{C}(35) \\
& C(25)-C(26)-C(29)-C(35) \\
& \mathrm{C}(28)-\mathrm{C}(26)-\mathrm{C}(29)-\mathrm{C}(35) \\
& C(30)-C(29)-C(31)-O(8) \\
& C(35)-C(29)-C(31)-O(8) \\
& \mathrm{C}(26)-\mathrm{C}(29)-\mathrm{C}(31)-\mathrm{O}(8) \\
& C(30)-C(29)-C(31)-C(32) \\
& \mathrm{C}(35)-\mathrm{C}(29)-\mathrm{C}(31)-\mathrm{C}(32) \\
& \mathrm{C}(26)-\mathrm{C}(29)-\mathrm{C}(31)-\mathrm{C}(32) \\
& \mathrm{O}(8)-\mathrm{C}(31)-\mathrm{C}(32)-\mathrm{C}(33) \\
& \mathrm{C}(29)-\mathrm{C}(31)-\mathrm{C}(32)-\mathrm{C}(33) \\
& \mathrm{C}(31)-\mathrm{C}(32)-\mathrm{C}(33)-\mathrm{C}(34) \\
& \mathrm{C}(32)-\mathrm{C}(33)-\mathrm{C}(34)-\mathrm{O}(8) \\
& C(31)-C(29)-C(35)-C(36) \\
& C(30)-C(29)-C(35)-C(36) \\
& C(26)-C(29)-C(35)-C(36) \\
& \mathrm{O}(7)-\mathrm{C}(28)-\mathrm{C}(36)-\mathrm{C}(37) \\
& \mathrm{C}(26)-\mathrm{C}(28)-\mathrm{C}(36)-\mathrm{C}(37) \\
& \mathrm{O}(7)-\mathrm{C}(28)-\mathrm{C}(36)-\mathrm{C}(38) \\
& \mathrm{C}(26)-\mathrm{C}(28)-\mathrm{C}(36)-\mathrm{C}(38) \\
& \mathrm{O}(7)-\mathrm{C}(28)-\mathrm{C}(36)-\mathrm{C}(35) \\
& C(26)-C(28)-C(36)-C(35) \\
& C(29)-C(35)-C(36)-C(37) \\
& \mathrm{C}(29)-\mathrm{C}(35)-\mathrm{C}(36)-\mathrm{C}(38) \\
& \mathrm{C}(29)-\mathrm{C}(35)-\mathrm{C}(36)-\mathrm{C}(28) \\
& \mathrm{O}(2)-\mathrm{C}(9)-\mathrm{O}(1)-\mathrm{C}(8) \\
& \mathrm{O}(3)-\mathrm{C}(9)-\mathrm{O}(1)-\mathrm{C}(8) \\
& \mathrm{C}(7)-\mathrm{C}(8)-\mathrm{O}(1)-\mathrm{C}(9) \\
& \mathrm{C}(17)-\mathrm{C}(8)-\mathrm{O}(1)-\mathrm{C}(9) \\
& \mathrm{O}(2)-\mathrm{C}(9)-\mathrm{O}(3)-\mathrm{C}(7) \\
& \mathrm{O}(1)-\mathrm{C}(9)-\mathrm{O}(3)-\mathrm{C}(7) \\
& \mathrm{C}(6)-\mathrm{C}(7)-\mathrm{O}(3)-\mathrm{C}(9) \\
& C(8)-C(7)-O(3)-C(9)
\end{aligned}
$$

$-0.6(3)$

$0.8(3)$

$-1.3(3)$

$179.43(16)$

$0.1(3)$

179.38 (17)

-148.78 (16)

$32.0(2)$

$-38.9(2)$

141.82 (17)

$89.4(2)$

$-89.9(2)$

$32.52(16)$

-82.18 (19)

144.96 (15)

$-86.78(16)$

$158.52(16)$

$25.7(2)$

-162.03 (14)

$-40.8(2)$

92.31 (17)

-42.11 (19)

$79.1(2)$

-147.77 (16)

$77.64(17)$

-161.13 (17)

$-28.0(2)$

$177.07(15)$

$53.98(19)$

-60.65 (18)

$57.8(2)$

$-65.3(2)$

-179.92 (15)

$30.77(19)$

$152.43(17)$

$-36.6(2)$

$30.0(2)$

$-98.39(18)$

$140.67(17)$

$20.8(2)$

114.26 (18)

-133.18 (17)

$-6.6(2)$

$106.00(18)$

-124.70 (17)

$-12.1(2)$

113.28 (19)

-126.48 (18)

$-5.8(2)$

169.87 (19)

-10.4 (2)

$27.75(18)$

$-88.01(17)$

$167.16(19)$

$-12.6(2)$

$-94.05(17)$

$28.10(17)$ 
$\mathrm{C}(10)-\mathrm{C}(7)-\mathrm{O}(3)-\mathrm{C}(9)$

$\mathrm{C}(14)-\mathrm{C}(15)-\mathrm{O}(4)-\mathrm{C}(12)$

$C(13)-C(12)-O(4)-C(15)$

$\mathrm{C}(10)-\mathrm{C}(12)-\mathrm{O}(4)-\mathrm{C}(15)$

$\mathrm{O}(6)-\mathrm{C}(27)-\mathrm{O}(5)-\mathrm{C}(26)$

$\mathrm{O}(7)-\mathrm{C}(27)-\mathrm{O}(5)-\mathrm{C}(26)$

$\mathrm{C}(25)-\mathrm{C}(26)-\mathrm{O}(5)-\mathrm{C}(27)$

$\mathrm{C}(28)-\mathrm{C}(26)-\mathrm{O}(5)-\mathrm{C}(27)$

$\mathrm{C}(29)-\mathrm{C}(26)-\mathrm{O}(5)-\mathrm{C}(27)$

$\mathrm{O}(6)-\mathrm{C}(27)-\mathrm{O}(7)-\mathrm{C}(28)$

$\mathrm{O}(5)-\mathrm{C}(27)-\mathrm{O}(7)-\mathrm{C}(28)$

$\mathrm{C}(26)-\mathrm{C}(28)-\mathrm{O}(7)-\mathrm{C}(27)$

$\mathrm{C}(36)-\mathrm{C}(28)-\mathrm{O}(7)-\mathrm{C}(27)$

$\mathrm{C}(29)-\mathrm{C}(31)-\mathrm{O}(8)-\mathrm{C}(34)$

$\mathrm{C}(32)-\mathrm{C}(31)-\mathrm{O}(8)-\mathrm{C}(34)$

$\mathrm{C}(33)-\mathrm{C}(34)-\mathrm{O}(8)-\mathrm{C}(31)$
$139.44(14)$

$10.6(2)$

$13.0(2)$

$138.70(16)$

-167.43 (19)

$13.0(2)$

$93.69(16)$

$-28.37(17)$

$-139.53(14)$

-169.56 (19)

$10.0(2)$

$-27.53(18)$

$88.42(17)$

-138.57 (16)

$-12.6(2)$

$-10.9(2)$ 